\title{
Psychopathy: its association with early maladaptive schemas, risk-related behaviors and treatment outcome
}

Citation for published version (APA):

Chakhssi, F. (2013). Psychopathy: its association with early maladaptive schemas, risk-related behaviors and treatment outcome. [Doctoral Thesis, Maastricht University]. Maastricht University. https://doi.org/10.26481/dis.20131220fc

Document status and date:

Published: 01/01/2013

DOI:

10.26481/dis.20131220fc

Document Version:

Publisher's PDF, also known as Version of record

Please check the document version of this publication:

- A submitted manuscript is the version of the article upon submission and before peer-review. There can be important differences between the submitted version and the official published version of record.

People interested in the research are advised to contact the author for the final version of the publication, or visit the DOI to the publisher's website.

- The final author version and the galley proof are versions of the publication after peer review.

- The final published version features the final layout of the paper including the volume, issue and page numbers.

Link to publication

\footnotetext{
General rights rights.

- You may freely distribute the URL identifying the publication in the public portal. please follow below link for the End User Agreement:

www.umlib.nl/taverne-license

Take down policy

If you believe that this document breaches copyright please contact us at:

repository@maastrichtuniversity.nl

providing details and we will investigate your claim.
}

Copyright and moral rights for the publications made accessible in the public portal are retained by the authors and/or other copyright owners and it is a condition of accessing publications that users recognise and abide by the legal requirements associated with these

- Users may download and print one copy of any publication from the public portal for the purpose of private study or research.

- You may not further distribute the material or use it for any profit-making activity or commercial gain

If the publication is distributed under the terms of Article $25 \mathrm{fa}$ of the Dutch Copyright Act, indicated by the "Taverne" license above, 


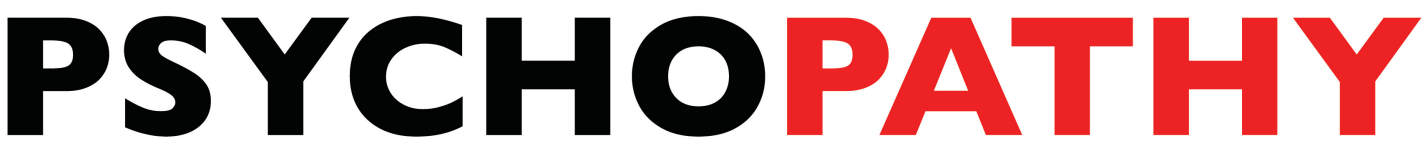

ITS ASSOCIATION WITH EARLY MALADAPTIVE SCHEMAS, RISK-RELATED BEHAVIORS \& TREATMENT OUTCOME 
Graphic design Jelske de Beer

Copyright @ 2013 by Farid Chakhssi

All rights reserved. No part of this publication may be transmitted or reproduced in any form by any means without the written permission of the copyright owner.

Published by Bureau Apeneus, Enschede (www.apeneus.nl) 
PSYCHOPATHY: ITS ASSOCIATION WITH EARLY MALADAPTIVE SCHEMAS, RISK-RELATED BEHAVIORS AND TREATMENT OUTCOME

PROEFSCHRIFT

ter verkrijging van de graad van doctor aan de Universiteit Maastricht, op gezag van de Rector Magnificus, Prof. dr. L.L.G Soete

volgens het besluit van het College van Decanen, in het openbaar te verdedigen

op vrijdag 20 december 2013 om 14.00 uur

door

Farid Chakhssi 
Promotores:

Prof. dr. C. de Ruiter

Prof. dr. D.P. Bernstein

Beoordelingscommissie

Prof. dr. H.L.G.J. Merckelbach, voorzitter

Prof. dr. A.R. Arntz

Prof. dr. H.L.I. Nijman, Radboud Universiteit Nijmegen

Prof. dr. Ch. van Nieuwenhuizen, Universiteit van Tilburg

Dr. V. de Vogel, Dr. Henri van der Hoeven Kliniek 
CHAPTER 1

General Introduction

\section{CHAPTER 2}

Reliability and Validity of the Dutch Version of the Behavioural Status Index: A Nurse-Rated Forensic Assessment Tool

\section{CHAPTER 3}

Early Maladaptive Schemas in Relation to Facets of Psychopathy and Institutional Violence in Offenders with Personality Disorders

$53 \quad$ CHAPTER 4

Early Maladaptive Cognitive Schemas in Child Sexual Offenders compared to Sexual Offenders against Adults and Nonsexual Violent Offenders

CHAPTER 5

Change during Forensic Treatment in Psychopathic versus Nonpsychopathic Offenders

85 CHAPTER 6

Treating the Untreatable: A Single Case Study of a Psychopathic Patient Treated with Schema Therapy

\section{CHAPTER 7}

General Discussion

121 Summary

127 Samenvatting (Dutch Summary)

135 References

157 Dankwoord

161 About the author 
This thesis is based on the following papers:

Chakhssi, F., de Ruiter, C., \& Bernstein, D. P. (2010). Reliability and validity of the Dutch version of the Behavioural Status Index: A nurse-rated forensic assessment tool. Assessment, 17, 158-169.

Chakhssi, F., Bernstein, D. P., \& de Ruiter, C. (2012). Early maladaptive schemas in relation to facets of psychopathy and institutional violence in offenders with personality disorders. Legal and Criminological Psychology. Article first published online: 8 November 2012.

Chakhssi, F., de Ruiter, C., \& Bernstein, D. P. (2013). Early maladaptive cognitive schemas in child sexual offenders compared to sexual offenders against adults and nonsexual violent offenders: An exploratory study. Journal of Sexual Medicine, 10, 2201-2210.

Chakhssi, F., de Ruiter, C., \& Bernstein, D. P. (2010). Change during forensic treatment in psychopathic versus nonpsychopathic offenders. Journal of Forensic Psychiatry \& Psychology, 21, 660-682.

Chakhssi, F., Kersten, G. C. M., de Ruiter C., \& Bernstein, D. P. (2014). Treating the untreatable: A single case study of a psychopathic inpatient treated with Schema Therapy. Psychotherapy, 51, 447-461. 


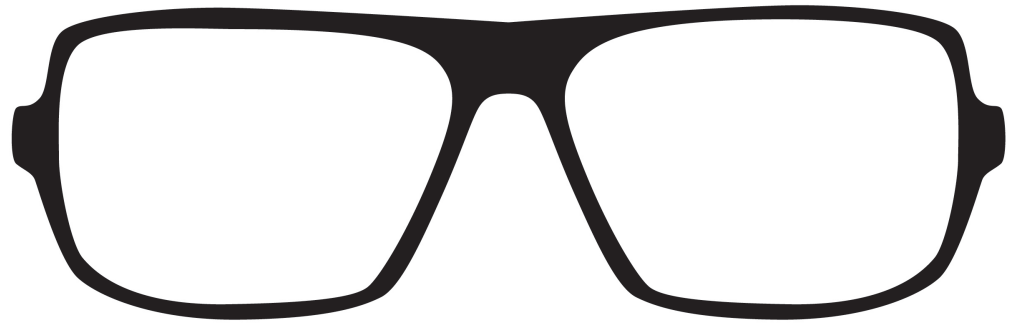




\section{INTRODUCTION}

Psychopathy is considered to be among the most important constructs in forensic psychiatry. Psychopathy is commonly defined as a personality disorder characterized by a manipulative interpersonal style, emotional detachment, impulsivity and persistent antisocial behavior (Hare, 2003). Whether persistent antisocial behavior should be considered as an essential part (Neumann, Hare, \& Newman, 2007) or a consequence (Cooke, Michie, \& Hart, 2006) of psychopathy is a topic of strong debate in the literature, while it remains unresolved (see Skeem, Polaschek, Patrick, \& Lilienfeld, 2011).

After imprisonment or treatment in a forensic psychiatric hospital, psychopaths are three to five times more likely to reoffend than nonpsychopaths (Hemphill, Hare, \& Wong, 1998; Leistico, Salekin, DeCoster, \& Rogers, 2008). Between 24\% and $35 \%$ of the population in Dutch inpatient forensic settings consists of psychopaths (Hildebrand, Hesper, Spreen, \& Nijman, 2005; Hildebrand \& de Ruiter, 2004), thus posing a considerable challenge for forensic treatment settings in safeguarding society. In particular, forensic mental health professionals are faced with the widely held belief that psychopaths are untreatable (Cleckley, 1941/1988) or that treatment makes them worse (see for instance, Hare, Clark, Grann, \& Thornton, 2000; Rice, Harris, \& Cormier, 1992; Seto \& Barbaree, 1999).

The notion that psychopathy is immutable has resonated long in the literature after the seminal work on psychopathy by Cleckley (1941) who observed that psychopaths did not seem to benefit from treatment nor were capable of forming the emotional bond required for effective therapy. Recent reviews, however, have led to the conclusion that there is little compelling empirical evidence that psychopathy is either immutable or amenable to treatment (e.g., D'Silva, Duggan, \& McCarthy, 2004; Harris \& Rice, 2006; Salekin, 2002; Salekin, Worley, \& Grimes, 2010). Earlier studies that fueled the clinical lore of psychopathy as untreatable suffered from methodological flaws, such as differing conceptualizations of the disorder, inappropriate treatments, lack of randomization and inadequate outcome measures (see D'Silva et al., 2004). Findings of a recent study in civil psychiatric patients suggest that patients with psychopathic traits were as likely as nonpsychopathic patients to benefit from psychological interventions (Skeem, Monahan, \& Mulvey, 2002; Skeem, 2008). Hence, this raises the possibility that psychological treatment may also be beneficial for forensic psychiatric patients with psychopathy.

In present-day forensic psychiatric practice, most interventions aimed at reducing the risk of reoffending are based on cognitive-behavioral theories of psychopathology and relapse prevention (Hollin \& Palmer, 2006). Recently, an innovative cognitive-behavioral therapy for personality disorders called Schema Therapy (Young, 1994) has shown to be promising in the treatment of disorders that were once considered difficult to treat, such as borderline personality disorder (e.g., Giesen-Bloo et al., 2006; Farrell, Shaw, \& Webber, 2009; Nordahl \& Nysaeter, 2005; Roper, Dickson, Tinwell, Booth, \& McGuire, 2010). Schema Therapy aims at reducing maladaptive personality traits and behavioral disturbances in severe personality disordered patients and could offer a potentially promising avenue in the treatment of psychopathic personality disorder (Bernstein, Arntz, \& de Vos, 2007). 
In Dutch forensic psychiatry, research into the treatment responsiveness of psychopathic patients is sparse (for an exception, see Hildebrand \& de Ruiter, 2012). This dissertation is aimed at contributing to the existing knowledge base by examining treatment responsiveness of offenders with psychopathic traits and by examining if the Schema Therapy model could be useful in the assessment and treatment of psychopathy. Hence, we will further elaborate on the concept of psychopathy and the cognitive framework of Schema Therapy.

\section{Conceptualization of psychopathy}

Most contemporary conceptualizations of psychopathy are based on the first clinical description of the psychopathic personality by Cleckley (1941). In his work The Mask of Sanity, Cleckley presented detailed case studies of several psychopathic patients. He described the psychopathic patient as a person marked by an outward appearance of robust mental health that masks a severe personality disturbance. Also, Cleckley introduced 16 specific characteristics that he posited as prototypical for the disorder, including superficial charm, lack of remorse or shame, and a general poverty in major affective reactions.

"The surface of the psychopath, however, that is, all of him that can be reached by verbal exploration and direct examination, shows up as equal to or better than normal and gives no hint at all of a disorder within. Nothing about him suggests oddness, inadequacy, or moral frailty. His mask is that of robust mental health" ( $p$. 383; Cleckley, 1988).

The diagnostic criteria provided by Cleckley (1941) served as the foundation for the development of Hare's Psychopathy Checklist (Hare, 1980). The revised version of the Psychopathy Checklist (PCL-R; Hare, 1991, 2003) has received widespread acceptance as a reliable and valid assessment tool of the clinical construct of psychopathy. The PCL-R consists of 20 items and each item is rated on a scale from 0 to 2 ( 0 = does not apply, 1 = applies to some extent, 2 = definitely applies). The PCL-R provides a dimensional total score between 0 and 40 indicating to what degree an individual matches the prototypical psychopath. The recommended cut-off score for classifying psychopathy in North-America is 30 (Hare, 2003). However, the cut-off score of 30 does not represent the same intensity of the disorder in European samples (Cooke, Michie, Hart, \& Clark, 2005). According to European studies, subjects with a total score of 26 or above (Hildebrand, 2004; Grann, Långström, Tengström, \& Kullgren, 1999) or with a PCL$\mathrm{R}$ total score of 28 or above (Cooke et al., 2005) can be classified as psychopathic.

Early factor-analytic studies suggest that the $20 \mathrm{PCL}-\mathrm{R}$ items reflect two underlying intercorrelated factors; the first factor represents emotional detachment whereas the second factor represents the socially deviant lifestyle and antisocial behaviors (Harpur, Hare, \& Hakstian, 1989). Recent studies, using structural equation modeling and Item Response Theory analysis, suggest that the two PCL-R factors can be broken down into three or four facets (Cooke, Michie, \& Skeem, 2007; Neumann et al., 2007; see Table 1). 
TABLE 1

PCL-R factors, facets and items (Hare, 2003)

\section{FACTOR 1: EMOTIONAL DETACHMENT}

\section{Interpersonal Facet}

Glibness/Superficial charm

Grandiose sense of self-worth

Pathological lying

Cunning/Manipulative

\section{Affective Facet}

Lack of remorse or guilt

Shallow affect

Callous/Lack of empathy

Failure to accept responsibility for own actions

\section{FACTOR 2: SOCIAL DEVIANCE}

\section{Antisocial Facet}

Poor behavioral controls

Early behavior problems

Juvenile delinquency

Revocation of conditional release

Criminal versatility

\section{Lifestyle Facet}

Need for stimulation/proneness to boredom

Impulsivity

Irresponsibility

Parasitic lifestyle

Lack of realistic long-term goals

Note. Items not included in factor or facet models: Promiscuous sexual behavior and Many short-term marital relationships. 
Psychopathy, as measured with the PCL-R, has been extensively studied to predict future violent behavior within institutional settings and within the community. For example, a recent meta-analysis based on 95 studies with nonoverlapping samples ( $N=15,826$; Leistico et al., 2008) which evaluated the association between PCL-R and violent behavior, including inpatient violence, showed moderate effect sizes (Hedges' $d$ ) for the PCL-R total score $(d=.55)$, Factor $1(d=.38)$ and Factor $2(d=.60)$.

Psychopathy appears to be a multifaceted disorder marked by differential relationships of the PCL-R facets with external criterion-related domains. For example, the Interpersonal facet of the PCL-R has demonstrated associations with instrumental violence, social dominance, low stress reactivity, and higher adaptive functioning (Hall, Benning, \& Patrick, 2004; Walsh, Swogger, \& Kosson, 2009). Deficient affective experience found in psychopaths, as reflected by the Affective facet, has been associated with neurobiological deficits in the limbic and other brain regions (Blair, 2003; Kiehl et al., 2001; Raine, Lencz, Bihrle, LaCasse, \& Colletti, 2000). The Lifestyle facet, including reactive aggression, has been shown to be associated with early traumatic experiences (Lang, af Klinteberg, \& Alm, 2002; Poythress, Skeem, \& Lilienfeld, 2006), serotonin dysfunction (Dolan \& Anderson, 2003) and suicidal behavior (Douglas et al., 2008; Verona, Patrick, \& Joiner, 2001). The Antisocial facet has been more strongly associated with crimerelated behavioral outcomes, such as institutional violence as well as, general and violent recidivism (i.e., relapse in offending), than the other facets (Kennealy, Skeem, Walters, \& Camp, 2010; Walters, Knight, Grann, \& Dahle, 2008; Walters \& Heilbrun, 2010).

\section{Treatment of psychopathy}

Since the conceptualization of the disorder by Cleckley (1941) and his observation that no treatment method impressed him as achieving successful results, psychopathy has been regarded by several prominent theorists (e.g., Kernberg, Yeomans, Clarkin, \& Levy, 2008; Reid \& Gacono, 2000; Skeem et al., 2002) as an incurable disorder. Despite the absence of randomized, controlled trials on treatment interventions for psychopathy, strong views still exist in the research literature that psychopathy is untreatable and/or that effective interventions for psychopathy do not exist (Harris \& Rice, 2006). In addition, findings from a few methodologically flawed studies (see D'Silva et al., 2004) have led to the policy of withholding treatment from psychopaths because psychopaths were supposedly 'made worse' by treatment. Two influential publications have contributed to the view that treatment makes psychopaths worse: the Penetanguishene therapeutic community study (Rice et al., 1992) and the evaluation of the Warkworth Sexual Behavior Clinic (Seto \& Barbaree, 1999).

The Penetanguishene therapeutic community study (Rice et al., 1992).

This study examined the effects of a therapeutic community (TC) implemented in a maximum-security forensic psychiatric hospital in the 1960s. Rice and colleagues (1992) assessed the outcome of this TC program and retrospectively scored the PCL-R based on the patients' chart data. They found that treated psychopaths, compared with matched untreated psychopaths drawn from a prison sample, were more likely to display violent recidivism ( $77 \%$ vs. $55 \%$ ) during an 
average follow-up period of 10.5 years compared to nonpsychopaths. In contrast, the treated nonpsychopaths were less likely to display violent recidivism during the same follow-up period compared to matched, untreated nonpsychopaths (22\% vs. $39 \%$ ), suggesting a positive effect of the therapeutic program on nonpsychopaths. These findings have been frequently offered to support the view that therapy has iatrogenic effects on the psychopath (Hare, 2002).

However, there are some serious limitations to this study, which prevent the establishment of firm conclusions that treatment makes psychopaths more dangerous. First, the therapeutic program may have been iatrogenic in itself. The program was described as a voluntary, peer-operated, intensive milieu therapy program with minimal contact of professional staff. Instead, patients who performed well were appointed to lead group therapy sessions, were given privileges to impose restrictions on other patients, and advised other patients to take medication. The program consisted of coercive structured interactions for at least 80 hours per week, and patients were prevented from leaving the activities by force or cuffs (Weisman, 1995). The therapeutic program did not include interventions that now are commonly used for reducing the risk of recidivism, such as altering pro-criminal attitudes and beliefs, teaching social skills or social problem solving, and training life skills. Second, the key components for therapeutic change were highly experimental, based on principles of brainwashing in a Chinese prison camp (Harris, Rice, \& Cormier, 1994) and judged by the authors themselves as violating patients' rights by today's standards (Rice et al., 1992). For example, one component was "defense-disruptive therapy" in which patients were coercively administered hallucinogenic drugs (i.e., scopolamine, LSD) with the goal of breaking through the patient's defense mechanisms and stimulating personal change (Barker, Mason, \& Wilson, 1969; Barker \& Buck, 1977). Another example was the "total encounter capsule" as the ultimate therapeutic tool for breaking down the psychopath's social mask and bringing about personal change. Here, patients spent at least two weeks nude in a windowless, continuously lighted, soundproofed, self-contained room deprived of any contact with the outside world (Barker \& McLaughlin, 1977). Third, other than by means of official recidivism data, the TC program was not evaluated. For example, there was no measure of change in clinical outcomes (e.g., psychopathology), and more importantly, there was no measure of change in the main focus of the program: psychopathic traits. This leaves open the possibility that other factors may have determined the outcome.

Finally, the most prevalent disorders among the offenders admitted to the Penetanguishene therapeutic community during the 1960s were either psychopathic personality or schizophrenia (Weisman, 1995). Rice and colleagues (1992) reported a significant difference $\left(\chi^{2}=36.04, p<.001, p .404\right)$ in the prevalence of schizophrenia between the TC treatment sample and the comparison prison only sample ( $27 \%$ vs. $2 \%$, respectively), indicating a selection bias. More importantly, schizophrenic patients are less likely to recidivate after hospitalization compared to other mentally disordered offenders (Bonta, Law, \& Hanson, 1998). However, the effect of the Penetanguishene TC treatment, after controlling for the difference in schizophrenia prevalence on recidivism rates, was not assessed. Hence, the "positive" effect of the therapeutic program in the nonpsychopathic patients may have been confounded by the differences in sample composition. 
The evaluation of the Warkworth Sexual Behavior Clinic

Seto and Barbaree (1999) evaluated a sex offender treatment program located in a medium-security federal penitentiary in the early 90 s. The PCL-R was scored as part of the standard assessment of the program. For the purpose of the study, the authors retrospectively scored treatment behavior from clinical notes and treatment reports, such as level of session participation, disruptiveness during sessions, homework quality, and clinical impressions of motivation and change. They found that psychopathic sex offenders who showed positive in-session behavior were more likely to seriously reoffend during an average follow-up period of 23 months, compared to psychopathic sex offenders who showed poor in-treatment behavior (20.4\% vs. $3.6 \%)$.

Several years later, Barbaree (2005) reexamined the same sample using a longer follow-up period ( $M=5.2$ years) and examined serious recidivism after fixed follow-up periods of 3, 5, and 6 years. In contrast to the Seto and Barbaree (1999) finding, there was no significant difference in serious recidivism rates between psychopathic sex offenders who showed positive in-session behavior compared to psychopathic sex offenders who showed poor in-treatment behavior (34\% vs. 30\%). Also, psychopathic patients were more likely to recidivate after 5 and 6 years, but not at 3 years. Neither treatment behavior nor the psychopathy-treatment behavior interaction was significant at any of the fixed follow-up times. Barbaree (2005) concluded there was no evidence that justified the earlier conclusion that treatment made psychopaths worse. He also stressed the importance of awaiting an accumulation of evidence over a number of studies before making major changes in policy and practice (Barbaree, 2005).

Treatment approaches for psychopathic offenders are still in their infancy. To date, no randomized controlled trials of treatments for psychopathy have been published in the clinical literature. Most existing approaches are cognitivebehavioral in nature (Hollin \& Palmer, 2006; Lösel \& Schmucker, 2005) and assume that the personality features of psychopathy cannot be changed; instead, they focus on helping psychopaths compensate for their emotional deficits by addressing their antisocial cognitions, teaching them more effective coping skills and enhancing their motivation towards prosocial goals and behaviors (Wong \& Hare, 2005).

In the small evidence base of research using structured assessments of psychopathy with PCL-versions, there is some evidence that psychological treatment may be helpful to psychopathic offenders. For example, in an evaluation of treatment in 871 civil psychiatric patients (Skeem et al., 2002), psychopathy did not moderate the effect of treatment involvement and subsequent violence during a post-discharge follow-up of 1 year. Similar findings were reported for a sample of 156 PCL-R assessed sex offenders. After a 10-year post-treatment follow-up, sex offenders who demonstrated positive therapeutic responses during a cognitivebehavioral orientated program with a relapse prevention component were less likely to recidivate in violent and sexual crimes (Olver \& Wong, 2009), regardless of their psychopathy scores.

In conclusion, there is no good evidence that psychopathy can be treated reliably and effectively, neither is there good evidence that it cannot be treated reliably and effectively (see also Salekin, 2002; Salekin et al., 2010). In order to 
further the understanding, assessment, and treatment of psychopathy, forensic mental health services are in need of more pilot studies, more case studies, more treatment effectiveness studies, and a variety of approaches to the assessment, and treatment of psychopathy (Hart, 2011).

\section{Schema Therapy}

Schema Therapy (ST; Young, Klosko, \& Weishaar, 2003) was specifically developed for patients who have been considered difficult to treat with traditional cognitive therapy. Often, patients with severe personality disorders fail to respond to, or relapse from, traditional cognitive therapy (Beck, Freeman, et al., 1990; Young, 1994). ST expands on the cognitive-behavioral approach developed by Beck, Freeman and colleagues (1990) by placing much greater emphasis on exploring the childhood origins of psychological problems, on experiential techniques, on the therapist-client relationship, and on maladaptive coping styles (Young et al., 2003). ST has shown effectiveness in three clinical trials of non-forensic outpatients with severe personality disorders, including two randomized controlled trials (Farrell et al., 2009; Giesen-Bloo et al., 2006) and one open trial (Nadort et al., 2009). ST has been recently adapted for forensic patients (Bernstein et al., 2007), and preliminary findings from an ongoing randomized clinical trial indicate that ST holds promise to be relevant for the assessment and treatment of offenders with personality disorders, including psychopathic ones (Bernstein et al., 2012).

One of the main targets of ST is change of chronic emotional and cognitive maladaptive patterns that originate in adverse childhood experiences and early temperament called Early Maladaptive Schemas (EMS). EMS are hypothesized to develop during negative childhood experiences - such as repeatedly being abused, neglected, abandoned, or rejected as a child - and are considered broad organizing principles for making sense of one's life experiences. In adulthood, EMS are triggered by life events that individuals (unconsciously) perceive as similar to their adverse childhood experiences, affecting emotional processing, influencing interpersonal interactions, and guiding behavior (Young et al., 2003). For example, maltreatment during childhood may lead to a Mistrust/Abuse Schema. Individuals with this schema have the belief that, given the opportunity, others will abuse, cheat, lie to, or manipulate them for their own needs.

EMS can be assessed with the Young Schema Questionnaire (YSQ; Young \& Brown, 1994; Dutch version: Sterk \& Rijkeboer, 1997). The Dutch version of the YSQ has shown adequate reliability and validity (Rijkeboer \& van den Bergh, 2006; Rijkeboer, van den Bergh, \& van den Bout, 2005).

\section{Early maladaptive schemas and psychopathy}

One of the first studies relating early childhood trauma with psychopathic traits was John Bowlby's classical work on 44 juvenile thieves (Bowlby, 1944). Bowlby studied a group of 44 juvenile offenders and compared them with 44 juvenile problematic, but non-offending, controls. Fourteen of the juvenile offenders were classified as 'affectionless psychopaths,' characterized by lack of concern for others, lack of guilt, shame, or sense of responsibility; whereas, none of the controls were similarly classified. Most of the affectionless psychopaths had a history of adverse childhood experiences that were hypothesized to contribute to the psychopathic 
traits. The few PCL-based studies that examined childhood trauma in relation to psychopathic traits in adulthood report generally consistent findings (Farrington, 2006; Lang et al., 2002; Weiler \& Widom, 1996). Similar findings are reported from studies in offender samples in which self-reported childhood trauma is associated with higher PCL-R scores (Graham, Kimonis, Wasserman, \& Kline, 2012; Koivisto \& Haapasalo, 1996; Marshall \& Cooke, 1999; Poythress et al., 2006; WeizmannHenelius et al., 2010). There is also some indirect evidence from longitudinal studies examining the interaction between genetic and environmental influences that childhood abuse is a strong risk factor for antisocial behavior in adulthood (e.g., Caspi et al., 2002; Huizinga et al., 2006). The relationship between childhood abuse and antisocial behavior was moderated, but not limited by genetic traits predisposing individuals towards impulsive and aggressive behavioral problems (Caspi et al., 2002). In other words, early adverse experiences are a risk factor for developing antisocial behavior in adulthood for both individuals with and without the genetic vulnerability towards such behavior. To our knowledge, no study has examined EMS in psychopathic offenders. However, the cognitive-emotional sequelae of adverse childhood experiences, in terms of EMS, may be a promising theoretical model for the assessment and treatment of psychopathy.

\section{Early maladaptive schemas and sexual offending}

Psychopathy plays an important role in violent offending (Leistico et al., 2008), and in combination with deviant sexual preferences, increases the risk of sexual recidivism (Hildebrand, de Ruiter, \& de Vogel, 2004; Olver \& Wong, 2006, Rice \& Harris, 1997). Sexual reoffending during and/or after forensic psychiatric treatment has a significant impact on society. This is especially true for sexual offending against children, where in the past a single incident has led to a societal and political debate on the quality of forensic psychiatric treatment in The Netherlands (Committee Visser, 2006). In the sexual offending literature, cognitions have been identified as key areas in the assessment of risk for future sexual offences (Hanson \& Morton-Bourgon, 2004) and as a major target in most treatment programs of sexual offenders (e.g., Marshall, Marshall, Serran, \& O'Brien, 2011). However, the theoretical role of cognitions in sexual offending is often poorly understood (ó Ciardha \& Gannon, 2011) and varies from post-hoc rationalizations for sexual offending behaviors to deep underlying, causal cognitive schemas (Maruna \& Mann, 2006). EMS may be valuable in clarifying the role of cognitions in sexual offenders (Marshall et al., 2011). It has been hypothesized that EMS, in interaction with sexual deviance, may increase the likelihood of sexually assaultive behavior towards children (Mann \& Beech, 2003; Marshall et al., 2011). Indeed, there is some evidence from longitudinal studies that childhood adversity is associated with sexual offending towards children in adulthood (Salter et al., 2003; Widom \& Ames, 1994). More specifically, experiences of being neglected and abandoned as a child were more related to sexual offending towards children in adulthood than other types of adverse experiences, including sexual abuse (Salter et al., 2003). These findings suggest that the cognitive-emotional sequelae of adverse childhood experiences, in terms of EMS, may also play a role in sexual offending. To our knowledge, no study has yet assessed EMS in adult sexual offenders. Studying 
EMS in sexual offenders may inform the assessment and treatment of sexual offenders.

\section{Measuring change}

The primary focus of forensic psychiatric treatment is the reduction in the risk of future (violent) offending in mentally disordered offenders. The ability to measure change in recidivism risk in a reliable and valid manner is a necessity in order to adequately guide decisions about treatment and risk management strategies. In most treatment outcome research, self-report is used as a method of measuring clinical change. However, in forensic settings, self-report methods need to be used with caution. In order to influence decisions about their treatment and release, forensic patients are prone to use impression management (Rogers, 2008), such as deliberately minimizing or denying psychiatric symptoms (e.g., Cima et al., 2003). To address these challenges in measuring change, several studies have focused on staff ratings of forensic patients' aggressive behavior (Hornsveld, Nijman, Hollin, \& Kraaimaat, 2007; Nijman, Evers, Merckelbach, \& Palmstierna, 2002) and psychiatric condition (Timmerman, Vastenburg, \& Emmelkamp, 2001). However, well-validated instruments for observing a broad range of risk-related behaviors in forensic psychiatric populations are scarce. Hence, an integral part of this research will involve validating a broad-spectrum observational measure for violence risk-related indicators.

\section{Setting of the present study}

The research reported in this thesis was conducted at Forensic Psychiatric Center (FPC) de Rooyse Wissel, one of the 13 maximum security forensic hospitals in The Netherlands. FPC de Rooyse Wissel offers treatment for mentally disordered offenders who are hospitalized under the Dutch judicial measure of TerBeschikkingStelling (TBS). A TBS-order can be imposed on offenders who have committed a serious offense, carrying a punishment of at least 4 years imprisonment, and who suffer from a mental disorder or a defective mental development. The TBS-order is prolonged by court as long as the patient is deemed a danger to society (de Ruiter \& Hildebrand, 2003).

FPC de Rooyse Wissel opened its doors for its first admissions in the year 2000. In 2012, the hospital had more than 200 residential treatment beds for male offenders, divided over two geographical locations, Venray and Maastricht. The overall treatment objective of de Rooyse Wissel is to reduce future violence risk in mentally disordered offenders by providing inpatient treatment and, in most cases, a stepwise reintegration into society with the aid of other correctional- and mental health- service providers. The treatment program is tailored to four offender groups: (1) offenders with schizophrenia and other psychotic disorders, (2) personality disordered offenders, (3) sex offenders and (4) intellectually disabled offenders.

Recently, the first follow-up study of patients treated at FPC de Rooyse Wissel was completed (Chakhssi \& Verwaaijen, 2012). Following the standards for tracking recidivism of the Dutch Ministry of Justice (Bregman \& Wartna, 2010), patients ( $N=$ 26) discharged from de Rooyse Wissel between 2004 and 2008 were followed-up. After the TBS-order had ended, most patients (89\%) did not recidivate in serious violent and/or sexual offenses (average follow-up $=5$ years). There were insufficient 
PCL-R scores available to perform further analyses examining the association between PCL-R scores and recidivism after discharge from the hospital.

\section{Aims and outline of the present thesis}

The present dissertation contains five empirical studies organized in two parts:

\section{Part I: Assessment}

The main goals of the empirical research reported in this part are:

1. To examine the reliability and validity of a nurse rated forensic assessment tool, the Dutch version of the Behavioural Status Index (BEST-Index), in a sample of male forensic psychiatric inpatients;

2. To explore the relationship between early maladaptive schemas and PCL-R facets in a sample of male personality disordered patients;

3. To explore the relationship between early maladaptive schemas and psychopathy in male child sexual offenders by comparing these patients with male sexual offenders against adults and male nonsexually violent offenders.

\section{Part II: Treatment}

The objectives of the studies in the second part are:

4. To examine change during long-term inpatient forensic treatment in a sample of male offenders with high and low scores according to Hare's PCL-R;

5. To explore the process of long term ST treatment, with a three-year follow-up post treatment, of a male forensic patient with prominent psychopathic features.

In Chapter 2, a study is reported that examines the psychometric properties of the Behavioural Status Index (BEST-Index; Reed, Woods, \& Robinson, 2000). The BESTIndex was introduced into Dutch forensic psychiatry to measure change in recidivism risk. Thus far, limited information regarding the psychometric properties of the Dutch version of the BEST-Index is available. Chapter 3 investigates the hypothesis that EMS are related to psychopathic traits and institutional violence in adult male personality disordered offenders. In Chapter 4, we examine if EMS can differentiate between child sexual offenders, sexual offenders against adults, and nonsexually violent offenders when controlling for level of psychopathy.

Chapter 5 compares change during forensic treatment between psychopathic and nonpsychopathic offender-patients. The offenders were assessed six-monthly over a two-year course by means of the BEST-Index, on adaptive social behavior, communication skills, insight, attribution of responsibility and self-regulation strategies. Change was examined at the individual- and group-level. Chapter 6 reports a detailed case study of Schema Therapy (ST) of a male forensic patient with psychopathic features. The case study includes independent assessments of multiple standardized outcome measures; the outcome is presented using reliable change and a three-year post-ST follow-up is included.

Finally, Chapter 7 summarizes the findings of this study. The strengths and limitations of the empirical studies of this thesis are discussed, as are suggestions for future research and the implications for forensic-clinical practice. 
18 | CHAPTER 1 
Reliability and Validity of the Dutch Version of the Behavioural Status Index: A Nurse-Rated Forensic Assessment Tool

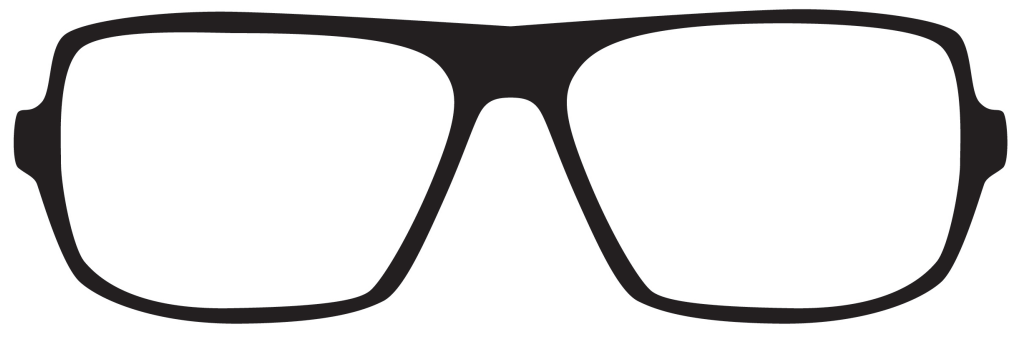




\section{SUMMARY}

The Behavioural Status Index (BEST-Index) has been introduced into Dutch forensic psychiatry to measure change in risk level of future violence. The BEST-index is a structured observational measure that assesses aggressive behavior, degree of insight, social skills, self-care, and work- and leisure skills during inpatient treatment. Thus far, limited information regarding the psychometric properties of the Dutch version of the BEST-Index is available. The present study examines the reliability and validity of the Dutch BEST-Index, in a sample of 291 mentally disordered offenders admitted to a forensic psychiatric hospital. Interrater reliability was investigated in a sample of 182 raters. Findings show that the Dutch BESTIndex can be used reliably and is significantly associated with risk of future violence and institutional aggression. Furthermore, this study revealed a different and clearer factor structure compared to the original one. Further research is needed to examine how these derived factors predict future recidivism.

\section{INTRODUCTION}

The primary focus of treatment of offender-patients in forensic psychiatric settings is the reduction of the risk of future (violent) offending. The ability to measure change in risk level of future violence is a prerequisite in order to adequately manage risk in mentally disordered offenders (Douglas \& Kropp, 2002). An individual's violence risk level may be seen as changing over time, depending on context and in response to interventions (Dvoskin \& Heilbrun, 2001; Heilbrun, 1997). Self-report is often used as a method of assessment in treatment outcome research. However, in forensic settings, self-report methods need to be used with prudence. Forensic patients are prone to use deception and impression management (Cima, 2003; Rogers, 1997) in order to influence decision making regarding release. To address these challenges with self-report, several observational measures have been developed, for example, for measuring aggression (Hornsveld, Nijman, Hollin, \& Kraaimaat, 2007; Nijman, Evers, Merckelbach, \& Palmstierna, 2002) and psychiatric condition (Timmerman, Vastenburg, \& Emmelkamp. 2001).

A particularly promising development in the forensic field is the design of instruments to assess change in recidivism risk based on objective risk factors. For example, the Historical Clinical Risk-management-20 (HCR-20; Webster, Douglas, Eaves, \& Hart, 1997), the most widely used and extensively validated of these measures, assesses both static (i.e., historical) and dynamic (i.e., changeable) risk factors for future violence (e.g., Strand \& Belfrage, 2001; de Vogel, de Ruiter, Hildebrand, Bos, \& Van de Ven, 2004; de Vogel \& de Ruiter, 2006) . The HCR-20 has shown good ability to predict moderate- to long-term recidivism (Douglas, Guy \& Weir, 2006). However, the HCR-20 was not originally intended to measure change in recidivism risk during forensic treatment (Douglas \& Skeem, 2005). Only 10 of the HCR-20's items measure dynamic risk factors, which may limit its suitability for 
measuring change in risk levels. For this reason, instruments are needed that focus primarily on assessing change in dynamic risk factors during forensic treatment.

Reed, Woods, and Robinson (2000) adapted the Behavioural Status Index (BEST-Index), a broad spectrum structured observational measure applied by nurses, for use in forensic psychiatric samples (Ross et al., 2008; Woods, 2000). The BEST-Index was first developed as an instrument for assessing change during general psychiatric treatment, and during patients' transition from a psychiatric hospital to community-based care (Mahgoub, 1988). For use in forensic settings, a risk scale was added to the BEST-Index and the original scales were modified for forensic use (Robinson, Reed, \& Lange, 1996). Recently, two subscales on selfcare and work skills were added (Reed et al., 2000). BEST-Index assessment is now specifically aimed at measuring five behavioral domains, including aggressive behavior, degree of insight, social skills, self-care, and work- and leisure skills.

Thus far, few studies have investigated the psychometric properties of the BEST-Index. Research on the factor structure of the BEST-Index in a sample of mentally disordered offenders $(N=503)$, yielded an ambiguous factor solution (Woods, Reed, \& Collins, 2005). In this study, the authors investigated the factor structure of the first three subscales: Risk, Insight and Social Skills, which resulted in an over-factored model consisting of 11 factors, where one factor had no salient loadings and one factor consisted of only one item. Other attempts to investigate the factor structure were based on the same sample and more explorative in nature. For example, factor analyses were limited to a single subscale (Woods, Reed, \& Collins, 2001a; Woods, Reed, \& Collins, 2001b; Woods, Reed, \& Collins, 2001c) or to two subscales (Woods, Reed, \& Collins, 2003a; Woods, Reed, \& Collins, 2003b; Woods, Reed, \& Collins, 2004). Although, these studies resulted in several factors per subscale or per two subscales, the identified structures did not emerge in a recent factor analytic study on the same sample containing all three subscales (Woods et al., 2005).

Internal consistency, test-retest stability and interrater reliability on the item level of the BEST-Index subscales Risk, Insight and Communication have been reported by Woods, Reed and Robinson (1999). Interrater reliabilities were weak to good, test-retest stabilities were good and, as a measure of internal consistency, item-to-subscale-correlations were weak to good. With regard to the convergent validity of the BEST-Index, statistically significant correlations were found between BEST-Index items and HCR-20 items (ranging from .41 to .44, Spearman $\rho$ correlations) in a forensic sample (Ross et al., 2008).

The possibility of using the BEST-Index as an inpatient observational measure of future violence risk served as an impetus to the introduction of the instrument in several Dutch forensic psychiatric hospitals. The instrument was translated into Dutch (van Erven, 1999) and nursing staff were trained in the use of the assessment tool. The present study examines the reliability and validity of the Dutch version of the BEST-Index in a sample of forensic psychiatric inpatients. First, interrater reliability of the BEST-Index total score and subscales was studied. Second, the internal reliability was examined by means of internal consistency and item homogeneity parameters. Third, the goodness-of-fit of the three original subscales of the BEST-Index was examined by means of confirmatory factor analysis. Because of inadequate fit (described later), the underlying latent 
constructs were investigated by means of principal components analysis. Finally, convergent and predictive validity was examined by measuring the association between the BEST-Index and the HCR-20 and institutional aggressive behavior.

\section{METHOD}

\section{Setting}

This study was conducted at Forensic Psychiatric Center 'de Rooyse Wissel' (dRW), a Dutch maximum security hospital for the treatment of mentally disordered offenders who were hospitalized under the Dutch judicial measure of "TerBeschikkingStelling" (TBS-order). A TBS-order can be imposed on offenders who have committed serious offences, carrying a punishment of at least 4 years imprisonment, and who suffer from a mental disorder according to the Diagnostic Manual and Statistical Manual of Mental Disorders, text revision (DSM-IV-TR) criteria (American Psychiatric Association, 2000). The TBS-order is prolonged as long as the court deems the patient a danger to society. The hospital has 229 residential treatment beds for male offenders, divided over three geographical locations.

\section{Sample}

The study sample consists of 291 male mentally disordered offenders, admitted to $\mathrm{dRW}$. These patients were admitted under the TBS-order during the period between March 1, 2000 and December 1, 2007. DSM-IV-TR Axis I and Axis II diagnoses were extracted from the patient files. These were based on clinical diagnoses made by psychiatrists, supported by findings from psychological assessments performed by clinical psychologists.

The characteristics of the study sample are presented in Table 1. Mean age was 38.2 ( $S D=9.5$ years). In the sample, $39.5 \%$ of the patients had committed (attempted) homicide, 23.3\% had committed sexual offenses, 23\% had committed violent theft, robbery or assault, $9.1 \%$ had committed arson and $1 \%$ had committed property offenses.

DSM-IV-TR Axis I and/or Axis II diagnoses were available for 287 patients. Classifications for four patients were deferred, awaiting additional (neuro-) psychological assessments. Patients were classified according to DSM-IV-TR Axis I disorder categories, if they met the diagnostic criteria for at least one disorder belonging to that category. Two-thirds of the patients $(65.9 \%)$ met the diagnostic criteria for at least one substance-related disorder. Seventy-one patients $(24.7 \%)$ met criteria for schizophrenia or other psychotic disorders. Fifty-seven patients (19.6\%) met criteria for a paraphilic disorder, 17 patients (5.9\%) for mood disorders, 19 patients (6.6\%) for anxiety disorders, 23 patients (8.0\%) for impulse control disorders, 21 patients (7.3\%) for pervasive developmental disorders, 25 patients $(8.7 \%)$ for attention deficit and disruptive behavior disorders and 14 patients (4.9\%) for other DSM-IV-TR Axis I disorder categories. In terms of Axis II personality disorders (PD), the most prevalent PD (56.1\%) was a mixed PD or PD Not Otherwise Specified (NOS). The prevalence of having any PD (including PD NOS) in this sample was $81.1 \%$ (236 patients). Subsequent to mixed PD and PD NOS, the most prevalent PD was antisocial PD (18.5\%). The other PDs were much less prevalent: three paranoid PD (1\%), two schizoid PD (.7\%), one schizotypical PD 
(.3\%), 16 borderline PD (5.6\%), four histrionic PD (1.4\%), 25 narcissistic PD (8.6\%), two avoidant PD (.7\%), and four obsessive-compulsive PD (1.4\%). No patient met the criteria for dependent PD.

\section{TABLE 1}

Sample Characteristics $(N=291)$

\begin{tabular}{lr}
\hline Characteristic & $38.2(S D=9.5)$ \\
\hline Age (years) & $39.4 \%$ \\
Main index offense & $23.3 \%$ \\
Homicide offense (including attempt) & $23.0 \%$ \\
Sexual offense & $1.0 \%$ \\
Violent theft, robbery, and assault & $9.1 \%$ \\
Property offense & \\
Arson & $80.1 \%$ \\
Criminal History & $22.8(S D=8.2)$ \\
$\quad$ Prior convictions & $8.2 \%$ \\
$\quad$ Age at first conviction & \\
$\quad$ Prior TBS & $17.6(S D=9.6)$ \\
Mental Health history & \\
$\quad$ Prior contact with mental health services $(n=287)$ & \\
$\quad$ Age at first mental health contact $(n=205)$ & \\
\hline
\end{tabular}

\section{Measures}

BEST-Index. The BEST-Index (Reed et al., 2000) contains 70 items divided a priori among three subscales: Risk, Insight, and Communication and Social Skills. Each item can be scored on a 5-point scale ranging from 1 (= worst case) to 5 (= optimal case). The Risk scale contains 20 items related to dangerous behaviors, such as violence to others, general disruptive and antisocial behaviors. The Insight scale consists of 20 items measuring the level of insight into: the nature of the patient's problems; antecedent events leading to their current situation; and attribution of responsibility. The Communication and Social Skills subscale contains 30 items on adaptive social behavior, social skills and interpersonal relationships. The BESTIndex is scored over the previous six-month period, and the time needed to complete an assessment varies between one and two hours. An example of an item, item description and the scoring categories are given in Table 2.

Test-retest reliabilities of the BEST-Index scales and items were satisfactory in an earlier study in a UK forensic psychiatric sample ( $N=100$; Woods et al., 1999). Reliabilities (Spearman $\rho$ correlations) over a two-week interval ranged, from .84 to .89 for the subscales and from .77 to .95 for the items. Item-to-subscale correlations (Spearman $\rho$ ) varied between .14 and .81 . Interrater reliabilities $(N=$ 
37; Spearman $\rho$ correlations) for the BEST-Index items varied from .03 to .77, with a mean of .43 for all items. In addition, items from the BEST-Index subscales Risk and Insight have been shown to be significantly associated (ranging from .41 to $.44, p \leq .01$; Spearman $\rho$ correlations) with the HCR-20 Clinical items Lack of insight; Negative attitudes; Active symptoms of major mental illness; Impulsivity; and Unresponsive to treatment as well as with the HCR-20 Risk-management item Lack of personal support (Ross et al., 2008). The BEST-Index was translated into Dutch (van Erven, 1999), in collaboration with the original authors, independent from the authors of the present study.

\section{TABLE 2}

Illustration of a BEST-Index item and scoring categories

\section{DESCRIPTION OF TENSION}

\begin{tabular}{lllll}
2.1 & 2.2 & 2.3 & 2.4 & 2.5 \\
Unable clearly & With support and & Occasionally & Usually & Always \\
to describe & encouragement & spontaneously & spontaneously & spontaneously \\
such feelings & occasionally & describes such & describes such & and clearly \\
& describes such & feelings & feelings & describes such \\
& feelings & reasonably clearly & reasonably clearly & feelings and their \\
& reasonably clearly & & & meanings \\
\hline
\end{tabular}

Note. Definition for Description of Tension: In conversation, the patient is able to describe and illustrate his/her experience whilst undergoing attacks of increased tension and nervousness.

HCR-20. The HCR-20 (Webster et al., 1997) is specifically designed for assessing the risk of future violence among persons with mental disorders. It consists of 20 items, each rated from 0 to $2(0=$ does not apply, $1=$ applies somewhat, 2 = definitely applies), divided among three scales: Historical $(\mathrm{H})$, Clinical $(\mathrm{C})$, and Risk Management (R). The $\mathrm{H}$ scale focuses on previous violent and antisocial behavior, the $\mathrm{C}$ scale on clinical aspects related to violence risk, and the $\mathrm{R}$ scale on future situational factors. In contrast to the 10 items of the $\mathrm{H}$ scale, the 5 items of the $\mathrm{C}$ scale and the 5 items of the $\mathrm{R}$ scale are amenable to change over time. The HCR-20 is intended to structure professional judgment about violence risk and raters are asked to make a final risk judgment of low, moderate or high (HCR20 manual; Webster et al., 1997). Research studies within diverse populations and in several countries have shown that the HCR-20 scores and final risk judgments can be derived reliably and are related to long-term violent recidivism (see Douglas et al., 2006).

START Outcome scale. The START Outcome scale is a modified version of the Overt Aggression Scale (OAS; Yudofsky, Silver, Jackson, Endicott, \& Williams, 1986) for use in forensic clinical practice (Nicholls, Brink, Desmarais, Webster, \& Martin, 2006). The modified version consists of 11 categories: (1) verbal aggression, (2) physical aggression against objects, (3) physical aggression against self, (4) physical aggression against other people, (5) self-neglect, (6) substance abuse, (7) 
victimized by others, (8) sexual aggression, (9) unauthorized leave, (10) suicidal behavior, and (11) stalking. Each category of adverse outcome is rated according to its severity on a 4-point scale from least severe (1) to most severe (4). Prior research shows that the START outcome scale can be reliably coded from patient files (intraclass correlation coefficient [ICC] = .70; Nicholls et al., 2006).

Raters and Training

BEST-Index raters were 182 psychiatric nurses (43.9\% male) with an average of 3.6 years of experience $(S D=2.5)$ at the $\mathrm{dRW}$ and a mean age of 35.8 years ( $S D$ $=8.8$ ). All raters attended a half-day workshop, given by the first author and/or a co-teacher (researcher or a master-level research-assistant). During the workshops, nurses were provided with the BEST-Index manual and informed about the nature and purpose of the BEST-Index. Second, the nurses were informed about the procedure for observational assessments of behavior. In addition, the nurses were presented with case vignettes and asked to rate several items of the BEST-Index. Finally, the scores were reviewed and discussed between the nurses and the trainer(s).

\section{Procedure}

Approval for the study was obtained from the hospital's executive board and the institutional research board (IRB).

BEST-Index assessments. Preferably, two psychiatric nurses assessed every patient using the BEST-Index six months after admission. Assessments were performed by at least the primary nurse on the ward where the patient resided, and one other nurse. On average, there were 4.7 days $(S D=8.0)$ between the two independent assessments. The scores were recorded in an electronic database with a web-based interface that had the same layout as the paper version of the BEST-Index. Scores from this database could be imported in the data-analysis software.

HCR-20 assessments. Ratings were performed by mental health professionals, who also coordinated the treatment of these patients. All the raters were trained and experienced in using the HCR-20. HCR-20 assessments are performed regularly when the TBS-order of the patient is evaluated (mostly every year, sometimes every 2 years). We conducted convergent validity analyses on patients who had at least one HCR-20 assessment $(n=224)$. For reliability analysis 48 cases were coded twice (by two trained Master-level psychology research-assistants), and the ICC for the HCR-20 total score was satisfactory at .76. For the individual HCR-20 scales, the ICCs were .72 for the H-scale, .75 for the C-scale and .66 for the R-scale (single measure ICCs, absolute agreement type).

START Outcome Scale assessments. Official registration of institutional misconduct was used to detect aggressive behaviors during treatment. Possible antecedents, motive, and a detailed description of the observed misconduct are registered by psychiatric nurses at each occurrence. To classify this misconduct in terms of type and severity, all misconducts during a patient's stay were recoded by three raters (a masters-level student and two masters-level research-assistants) with the English version of the START outcome scale (Nicholls et al., 2006). To ensure a prospective design, all misconducts that occurred prior to the first assessment of the BEST-Index were eliminated from the predictive analyses. 
Although coded retrospectively, due to the detailed official registration, this procedure is not likely to underestimate the actual prevalence of inpatient misconduct. More than three hundred incidents were coded by two raters, to permit reliability analysis. In this study, ICC for two raters was satisfactory at .83 (mean ICC; $n=369$ ).

\section{Statistical analysis}

Interrater reliability. For estimating the interrater reliability, the two-way random effects model, with measures of absolute agreement, of the ICC was used (Shrout \& Fleiss, 1979). Nurses in this study are a random sample from all possible raters, and patients are also a random factor. In this study, 262 patients were rated twice due to rater attrition for several reasons (e.g., absence of the second rater due to illness or leave; the patient was transferred to another ward while a second rating was pending). Interrater reliability for the BEST total and subscale scores as well as for the individual BEST-Index items was examined.

Internal consistency and item homogeneity. Internal consistency was examined using Cronbach's alpha for the BEST total score as well as for the subscales. Mean inter-item correlations were used as a measure of item homogeneity. Mean scores between raters, or when unavailable the scores of the single raters, were used for these and subsequent analyses.

Confirmatory factor analysis. The goodness-of-fit of the original three-factor model of the BEST-Index (Woods, 2000) was tested using confirmatory factor analysis. The model was fit using AMOS 7.0, employing the Maximum Likelihood procedure (Arbuckle, 2006). Each item was assumed to load only on its own subscale. Given the expected correlations between the subscales (Woods, 2000), the three subscales were allowed to correlate. Model fit was evaluated using the $\chi^{2}$ test, the Root Mean Square Error of Approximation (RMSEA), Comparative Fit Index $(\mathrm{CFI})$ and the Tucker Lewis Index (TLI; also known as the Non Normed Fit Index; Byrne, 2001). A CFI and TLI value above .90 and a RMSEA value below .05 are indicative for an adequate fit (Hu \& Bentler, 1999).

Principal components analysis with Varimax rotation. Because of an inadequate fit (described later), we examined the latent constructs underlying the BEST-Index using exploratory principal components analysis (PCA). The extracted communalities, which measure the percentage of variance in the variables explained by the extracted factors, were used to select variables to include in the analysis. Items with low communalities, meaning that the components explain little variance for these variables, are indicative of items that are unrelated to the domains of interest, and should be removed from further analyses (Fabrigar, Wegener, MacCallum, \& Strahan, 1999). Items with communalities below .20 were removed from further analyses. Finally, Varimax rotation was used to test the relative suitability of the resulting factor structure.

Convergent and predictive validity. Pearson product-moment correlations were used to measure the association between the BEST-Index scores and the level of violence risk (HCR-20). Convergent validity would be supported when the BEST-Index is stronger and statistically significant correlated with the dynamic risk factors of the HCR-20 (i.e., the C- and R-scale) compared to the static risk factors of the $\mathrm{H}$ scale. Further, predictive validity of the BEST-Index was examined by computing 
Pearson correlation coefficients between the BEST-Index scales and institutional adverse outcomes (START Outcome Scale). To test the BEST-Index relation to violence only the categories related to aggression to others will be used in this study. For the aggressive behavior, total score on START Outcome categories verbal aggression, physical aggression against objects, and physical aggression towards other people, was computed by summing the incidences for each category during a patient's stay (mean length of stay in hospital $=3.2$ years, $S D=2.2$ years). Analyses are performed using the Statistical Package for the Social Sciences, version 13.0 (SPSS Inc., 2005).

\section{RESULTS}

Confirmatory factor analysis. The goodness-of-fit indices for the original BESTIndex three-factor model did not meet the required cut-off values. The $\chi^{2}$ test yields a statistic of $7050.81(d f=2342 ; N=279)$, which has a corresponding $p$-value of .000. This $p$-value means that the null hypothesis of a good fit should be rejected. Additionally, the RMSEA is .09, the CFI is .65 and the TLI is .64, offering further evidence that the original model does not fit the present data well. In addition, inspection of the parameter estimates revealed that Risk subscale item 1 (critical ratio $=1.68, p=.093$ ) and item 16 (critical ratio $=1.59, p=.111$ ) did not significantly predict the Risk subscale, suggesting that removing these items could improve model fit. Also, the error terms covariances between items revealed that seven pairs of items had high modification indices $(>80)$. These items pairs were items 13-14, and 2-4 from the Risk subscale, and items 2-3, 13-14, 19-20, 19-23 and 20-23 from the Insight subscale. The high modification indices suggest that the model fit will improve, if the model is respecified by allowing these error terms to covary. A stepwise approach by first removing the Risk subscale Items 1 and 16 from the model and subsequently allowing the items with the highest modification indices to covary, resulted in a significantly improved model, that is, change in $\chi^{2}$ $(d f=142 ; N=279)=1094.5, p<.001$. However, the model still did not meet the cut-off values indicating a good model fit, $\chi^{2}(d f=2200 ; N=279)=5956.3, p=$ $.000, \mathrm{RMSEA}=.08, \mathrm{CFI}=.72$ and $\mathrm{TLI}=.71$. Thus, an exploratory PCA was deemed necessary to reveal the instrument's factor structure.

Principal components analysis. Initial principal components analysis (PCA) with Varimax rotation yielded 14 components with eigenvalues greater than one, accounting for $70.0 \%$ of the total variance. In addition, the scree plot shows a steep drop over the first four components, followed by a leveling off for the remaining components. The first four components accounted for $50.5 \%$ of the total variance. The first component accounted for $31.7 \%$ of the total variance, whereas the other three components accounted for $9.5 \%, 5.2 \%$ and $4.2 \%$, respectively. These findings suggest that the four components offer the optimal solution. The extracted communalities of the items for the four components demonstrated low communalities $(<.20)$ for seven items from the Risk subscale. These items and their communalities were: Risk Item 1 (Family support; .04), Item 6 (Serious selfharm; .08), Item 7 (Superficial self-harm; .10), Item 15 (Inappropriate sexual behaviors; .11), Item 16 (Sado-masochistic behaviors; .08), Item 17 (Macho gear and adornment; .05), and Item 19 (Substance abuse; .06). These items were subsequently removed. PCA with Varimax rotation on the remaining 63 items now 
yielded 11 components with eigenvalues greater than 1, accounting for $70.4 \%$ of the total variance. Again, the scree plot shows a steep drop over the first four components, followed by a leveling off for the remaining components. Now, the first four components accounted for $55.5 \%$ of the total variance. The first component accounted for $35.1 \%$ of the total variance, whereas the other three components accounted for $10.2 \%, 5.7 \%$, and $4.5 \%$, respectively. The pattern matrix with loadings after rotation, with the items sorted by the size of their loadings, is displayed in Table 3. Factor 1 comprises 23 items and seems to address Social Skills. It consists primarily of items from the original Social Skills subscale. The highest loadings were found for Social Skills Item 28 (Ease of communication; .76), Social Skills Item 23 (Assertiveness; .74), and Social Skills Item 19 (Expressing opinions; .73). The weakest loading was found for Social Skills Item 4 (Body posture; .49). Factor 2 consists of 21 items and seems to address Insight, as demonstrated by items primarily from the original Insight subscale. The highest loadings were found for Insight Item 16 (Self-appraisal; .76), Insight Item 2 (Description of tension; .76), and Insight Item 17 (Prioritization of problems; .75). The weakest loading was found for Insight Item 3 (Tension reducing strategies; .50). Factor 3 consists of 14 items and the highest loadings were found for Social Skills Item 13 (Turn-taking; .72), Risk Item 9 (Verbal aggression following trigger event; .71) and Social Skills Item 14 (Listening skills; .67).

The weakest loadings were found for Risk Item 14 (Imitative disruption; .46). These items are related to aggressive and dominant behavior in interpersonal communication and hence, Factor 3 was named Interpersonal Hostility. Finally, Factor 4 consists of seven items related to Physical Violence. The highest loadings were found for Risk Item 2 (Serious violence to others without apparent trigger event; .79), Risk Item 4 (Minor violence to others without apparent trigger event; .78), and Risk Item 3 (Serious violence to others following trigger event; .72). The weakest loadings were found for Risk Item 10 (Attacks on objects without apparent trigger event; .53).

The mean scores on the factors for the sample are displayed in Table 4. The mean scores were $85.34(S D=16.39)$ for the Social Skills factor, $56.42(S D=$ 15.63) for the Insight factor, $47.16(S D=7.59)$ for the Interpersonal Hostility factor, and $33.31(S D=2.91)$ for the Physical Violence factor. Internal consistency ${ }^{1}$ for the factors is also displayed in Table 4, and ranged from $\alpha=.74$ for the Physical Violence factor to $\alpha=.96$ for the Social Skills factor. Finally, homogeneity of the items ranged from .38 for the Interpersonal Hostility factor to .51 for the Insight factor. 


\section{TABLE 3}

Item loading of BEST-Index four factor solution with Varimax rotation

\begin{tabular}{|c|c|c|c|c|c|}
\hline \multicolumn{2}{|l|}{ BEST-Index } & \multicolumn{4}{|c|}{ Factor } \\
\hline Items & Description & 1 & 2 & 3 & 4 \\
\hline SOCIAL28 & Ease of communication & .76 & .34 & .15 & \\
\hline SOCIAL23 & Assertiveness & .74 & .16 & -.26 & \\
\hline SOCIAL19 & Expressing opinions & .73 & .27 & -.18 & \\
\hline SOCIAL20 & Disagreement & .72 & .15 & -.30 & \\
\hline SOCIAL22 & Making requests & .71 & .24 & & \\
\hline SOCIAL12 & Fluency & .71 & .35 & & \\
\hline SOCIAL16 & Conversational topics & .70 & .39 & .32 & \\
\hline SOCIAL10 & Conversational initiative & .69 & .40 & & \\
\hline SOCIAL11 & Amount of speech & .68 & .40 & .36 & \\
\hline SOCIAL29 & Sociability and support & .67 & .41 & .11 & \\
\hline SOCIAL1 & Facial expression & .66 & .28 & .20 & \\
\hline SOCIAL7 & Tone of voice & .66 & .29 & & \\
\hline SOCIAL25 & Social activities & .65 & .35 & & .11 \\
\hline SOCIAL5 & Expressive gestures & .64 & .29 & .12 & \\
\hline SOCIAL2 & Eye contact & .62 & .30 & .27 & \\
\hline SOCIAL9 & Verbal delivery & .58 & .16 & .31 & \\
\hline SOCIAL15 & Response to questions & .58 & .35 & .41 & \\
\hline SOCIAL3 & Orientation to others & .57 & .27 & .28 & \\
\hline SOCIAL24 & Self-presentation & .56 & .16 & .14 & \\
\hline SOCIAL26 & Emotional control & .53 & .19 & .47 & .20 \\
\hline SOCIAL27 & Relationship with others & .53 & .28 & .42 & \\
\hline SOCIAL8 & Voice modulation & .51 & .14 & .35 & \\
\hline SOCIAL4 & Body posture & .49 & .17 & .38 & \\
\hline INSIGHT6 & Self-appraisal & .19 & .76 & .29 & \\
\hline INSIGHT2 & Description of tension & .24 & .76 & & \\
\hline INSIGHT17 & Prioritization of problems & .17 & .75 & .31 & \\
\hline INSIGHT6 & Tension-producing events & .31 & .74 & & \\
\hline INSIGHT1 & Awareness of tension & .19 & .73 & & \\
\hline INSIGHT5 & Tension-producing thoughts & .28 & .73 & & .13 \\
\hline INSIGHT12 & Events producing insecurity & .30 & .72 & & \\
\hline INSIGHT4 & Recognition of negative feelings & .25 & .70 & -.22 & \\
\hline INSIGHT14 & Antecedent events leading to treatment & .15 & .69 & .23 & \\
\hline INSIGHT15 & Ascription of responsibility & .13 & .68 & .32 & \\
\hline INSIGHT18 & Goal planning & .25 & .68 & .37 & \\
\hline INSIGHT2O & Expectations & .25 & .67 & .40 & \\
\hline INSIGHT8 & Identifying relaxing thoughts & .34 & .64 & .15 & .14 \\
\hline
\end{tabular}


TABLE 3 (continued)

\begin{tabular}{|c|c|c|c|c|c|}
\hline \multicolumn{2}{|l|}{ BEST-Index } & \multicolumn{4}{|c|}{ Factor } \\
\hline Items & Description & 1 & 2 & 3 & 4 \\
\hline INSIGHT13 & Events producing security & .44 & .62 & .12 & \\
\hline INSIGHT7 & Personal strategy for reducing tension & .32 & .61 & .13 & .16 \\
\hline INSIGHT11 & Attributes liked in others & .47 & .56 & & \\
\hline INSIGHT9 & Identifying relaxing activities & .39 & .56 & & \\
\hline SOCIAL18 & Frankness & .34 & .55 & .19 & \\
\hline INSIGHT10 & Attributes disliked in others & .44 & .52 & -.17 & .13 \\
\hline INSIGHT19 & Compliance with therapy & .28 & .51 & .42 & .14 \\
\hline INSIGHT3 & Tension-reducing strategies & .34 & .50 & & .12 \\
\hline SOCIAL13 & Turn-taking & & & .72 & \\
\hline RISK9 & Verbal aggression following trigger event & -.13 & & .71 & .43 \\
\hline SOCIAL14 & Listening skills & .40 & .14 & .67 & \\
\hline SOCIAL17 & Egocentric conversation & .24 & .14 & .67 & \\
\hline SOCIAL21 & Arguments & .20 & .12 & .62 & .33 \\
\hline RISK13 & Disruptive episodes & -.19 & & .58 & .17 \\
\hline SOCIAL6 & Social distance & .31 & .11 & .57 & .11 \\
\hline SOCIAL30 & Deferring to others & .17 & .30 & .55 & \\
\hline RISK8 & Verbal aggression without apparent trigger event & & & .54 & .52 \\
\hline RISK18 & Obsessive compulsive behaviors & & & .49 & .48 \\
\hline RISK20 & Psychiatric disturbance & .29 & & .47 & .12 \\
\hline RISK14 & Imitative disruption & & & .46 & .20 \\
\hline RISK2 & $\begin{array}{l}\text { Serious violence to others without apparent trigger } \\
\text { event }\end{array}$ & & .11 & & .79 \\
\hline RISK4 & Minor violence to others without apparent trigger event & & & & .78 \\
\hline RISK3 & Serious violence to others following trigger event & & & .11 & .72 \\
\hline RISK11 & Attacks on objects following trigger event & .10 & & .26 & .63 \\
\hline RISK5 & Minor violence to others following trigger event & & & .19 & .60 \\
\hline RISK12 & Breaches of security & & & .42 & .56 \\
\hline RISK10 & Attacks on objects without apparent trigger event & .17 & & .16 & .53 \\
\hline Proportion o & ariance explained per factor (\%) & 35.1 & 10.2 & 5.7 & 4.5 \\
\hline
\end{tabular}

Note. factor loadings $<.10$ are suppressed. Salient loadings are in bold.

Interrater reliability. The single measure ICC based on absolute agreement for the BEST-Index total score containing 63 items was .71. For the BEST-Index factors extracted in the present study (Table 3), the coefficients were .71 (Social Skills factor), .66 (Insight factor), .69 (Interpersonal Hostility factor), and .68 (Physical Violence factor), respectively. The coefficients for the individual items varied from .31 to .63 (median $=.50$ ) for the Social Skills factor, from .35 to .65 (median = .47 ) for the Insight factor, from .35 to .73 (median $=.44$ ) for the Interpersonal Hostility factor, and from .12 to .66 (median $=.47$ ) for the Physical Violence factor. 
The lowest single measure coefficient was obtained for Item 2 of the Risk subscale (Serious violence to others without apparent trigger event; .12). Item 9 of the Risk subscale (Verbal aggression following trigger event; .73) obtained the highest coefficient. $^{2}$

\section{TABLE 4}

Labels, mean scores, Cronbach's alpha, item homogeneity and interrater reliability the BEST-Index fourfactor solution

\begin{tabular}{|c|c|c|c|c|c|}
\hline \multirow[t]{2}{*}{ Scale } & \multicolumn{5}{|c|}{ Total sample $(N=291)$} \\
\hline & $i$ & $\alpha$ & Mean (SD) & Item homogeneity & $\mathrm{ICC}^{\mathrm{a}}$ \\
\hline Revised BEST total ${ }^{b}$ & 63 & .97 & $222.69(34.74)$ & .31 & .71 \\
\hline Social Skills & 23 & .96 & $85.34(16.39)$ & .49 & .71 \\
\hline Insight & 21 & .95 & $56.42(15.63)$ & .51 & .66 \\
\hline Interpersonal Hostility & 12 & .86 & $47.16(7.59)$ & .38 & .69 \\
\hline Physical Violence & 7 & .74 & 33.31 (2.91) & .40 & .68 \\
\hline
\end{tabular}

\section{TABLE 5}

Pearson correlation coefficients between the four factors of the BEST-Index and the HCR-20 scores $(n=$ 224)

\begin{tabular}{|c|c|c|c|c|c|}
\hline \multirow[t]{2}{*}{ Scale } & \multicolumn{5}{|c|}{ HCR-20 } \\
\hline & H Scale & C Scale & R Scale & Total Score ${ }^{c}$ & Risk judgment \\
\hline Revised BEST total ${ }^{a, b}$ & .01 & $.45 * *$ & $.42 * *$ & $.33 * *$ & $.34 * *$ \\
\hline Social Skills & .05 & $.31 * *$ & $.30 * *$ & $.21 * *$ & $.27 * *$ \\
\hline Insight & .09 & $.43 * *$ & $.44 * *$ & $.29 * *$ & $.35 * *$ \\
\hline Interpersonal Hostility & $.20 * *$ & $.41 * *$ & $.29 * *$ & $.38 * *$ & $.23 * *$ \\
\hline Physical Violence & $.21 * *$ & $.29 * *$ & $.15^{*}$ & $.28 * *$ & .13 \\
\hline Original BEST total & .00 & $.45 * *$ & $.42 * *$ & $.34 * *$ & $.34 * *$ \\
\hline Risk subscale & $.28 * *$ & $.40 * *$ & $.23 * *$ & $.39 * *$ & $.15^{*}$ \\
\hline Insight subscale & .09 & $.43 * *$ & $.44 * *$ & $.29 * *$ & $.35 * *$ \\
\hline Social Skills subscale & .01 & $.34 * *$ & $.32 * *$ & $.25 * *$ & $.29 *$ \\
\hline
\end{tabular}

Note. $* \mathrm{p}<.05, * * \mathrm{p}<.01$ (two-tailed). 
Convergent and predictive validity. In this sample, the mean HCR-20 score was 25.1 $(S D=6.19)$. For the HCR-20 scales, the mean score for the H scale was 13.19 (SD $=3.39)$, for the $C$ scale $4.78(S D=2.19)$, and $7.17(S D=2.59)$ for the $\mathrm{R}$ scale. Most patients ( $n=146 ; 65.1 \%$ ) received a final risk judgment of high risk, whereas $46(20.5 \%)$ patients were judged to pose a moderate risk and 32 (14.3\%) patients a low risk. Regarding the convergent validity of the BEST-Index, the correlations of the original BEST-Index and the revised BEST-Index (containing 63 items) with the HCR-20 are displayed in Table 5. From Table 5, it can be glanced that for the original BEST-Index, out of 20 possible correlations, 17 were significant. Significant correlations ranged from .15 (for the BEST-index Risk subscale and HCR-20 final risk judgment) to .45 (for the original BEST Index total score with HCR-20 C scale). For the revised BEST-Index, out of 25 possible correlations, 21 were significant. Significant correlations ranged from .15 (for the revised BEST-Index Physical Violence factor and HCR-20 R-scale) to .45 (for revised BEST-Index total score with HCR-20 C scale). Overall, the differences in the correlation matrix between the original and the revised BEST-index original were small, with the exception of the higher correlations between the revised BEST-index Interpersonal Hostility and Physical Violence factors with the Historical Scale of the HCR-20.

\section{TABLE 6}

Pearson correlation coefficients between the four factors of the revised BEST-Index and challenging and aggressive behaviors as measured with the START Outcome scale $(n=291)$

\begin{tabular}{llll}
\hline Scale & \multicolumn{3}{c}{ START Outcome scale } \\
\cline { 2 - 4 } & Verbal Aggression & $\begin{array}{l}\text { Physical Aggression } \\
\text { Against Objects }\end{array}$ & $\begin{array}{l}\text { Physical Aggression } \\
\text { Towards Others }\end{array}$ \\
\hline Revised BEST total & $.20 * *$ & $.15^{* *}$ & $.20^{* *}$ \\
Social Skills & .11 & .06 & $.13^{*}$ \\
Insight & .11 & .08 & .10 \\
Interpersonal Hostility & $.41 * *$ & $.27^{* *}$ & $.34^{* *}$ \\
Physical Violence & $.20 * *$ & $.25^{* *}$ & $.23^{* *}$ \\
& & & $.26 * *$ \\
HCR-20 total score ${ }^{\mathrm{a}}$ & $.32 * *$ & $.21^{* *}$ & $.27^{* *}$ \\
H-scale & $.29 * *$ & $.20^{* *}$ & $.21^{* *}$ \\
C-scale & $.27 * *$ & $.18^{* *}$ & .12 \\
R-scale & $.20 * *$ & .12 & \\
\hline
\end{tabular}

Note. ${ }^{\mathrm{a}} n=214 . * \mathrm{p}<.05, * * \mathrm{p}<.01$ (two-tailed).

During their stay, most of the patients ( $n=200 ; 68.7 \%)$ displayed acts of verbal aggression, 100 patients (34.4\%) displayed acts of physical aggression against objects, and 134 patients (46.0\%) displayed physical aggression towards others. The Pearson correlations between the revised BEST-Index scores, the HCR20 scores, and the START Outcome scales are presented in Table $6^{3}$. The highest correlation coefficient between the revised BEST-Index-scores and the START Outcome scales is found for the association between the Interpersonal Hostility 
factor and START verbal aggression $(r=.40 ; p<.01)$. The lowest, but still significant correlation coefficient is found for the relationship between physical aggression towards others and the Social Skills factor $(r=.13 ; p<.05)$. For the relationship between the HCR-20 and the START outcome scales the highest correlation coefficient is found for the H-scale and START verbal aggression $(r=$ $.30 ; p<.01)$. The lowest significant correlation is found for the association between the $\mathrm{C}$ scale and START physical aggression towards others $(r=.19 ; p<$ $.01)$.

\section{DISCUSSION}

The present study examined the reliability and factor structure of the Dutch version of the BEST-Index in a sample of 291 forensic psychiatric inpatients. Confirmatory factor analysis demonstrated that the original, a priori three-factor structure could not be replicated in our sample. In addition, two items had no significant contributions to the BEST-Index Risk subscale, and several item pairs of both the Risk and the Insight subscale had strong residual correlations. Correcting for these shortcomings resulted in a significantly improved model fit, but not to an acceptable overall model fit for the data in this sample. These findings are in line with the previous factor analytic study of the BEST-Index (Woods et al., 2005), which also did not support the original a priori factor structure.

The subsequent principal components analysis resulted in a four-factor solution, which primarily indicated that (1) the Risk and Insight subscales could be retained; (2) a number of items from the original Risk and Social Skills subscales relating to interpersonal dominance and aggressive behavior formed a new, third factor; and (3) the remaining items of the Risk subscale referring to physical violence also formed another, fourth factor. Seven items were omitted from the BEST-index because they were unrelated to any of the factors. Our findings are in contrast with earlier factor analytic work on the BEST-Index. Woods et al. (2005) identified 11 factors by selecting the factors solely on the basis of Kaiser's eigenvalue greater than 1 criterion. Although this procedure is appealing in its simplicity, exclusive reliance on this approach can lead to solutions with too many factors (over-factoring), which have little theoretical and/or practical value. In determining the optimal number of factors, several statistical (e.g., scree test) and theoretical procedures (e.g., conceptual clarity of the solution) should be employed (Fabrigar et al., 1999). Thus, the present study extended on the previous factor analytic studies by taking these considerations into account. Earlier factor analytic studies on the separate subscales (Woods et al., 2001a; Woods et al., 2001b; Woods et al., 2001c), and on two subscales combined (Woods et al., 2003a; Woods et al., 2003b; Woods et al., 2004) yielded different results in each instance, leading to a lack of conceptual clarity surrounding the factor structure of the BESTIndex. This is unfortunate because the BEST-index deserves a more systematic approach towards instrument development. Our findings obtained by this more systematic approach suggest that a four-factor model is a better solution than the original three-factor model.

The interrater reliabilities for the nurse raters were good for the four factors of the BEST-Index. These interrater reliabilities are consistent with those found in the 
only other interrater reliability study of the BEST-Index in a sample of 37 raters (Woods et al., 1999). Note that in the current study we had a sample of 182 raters and used the stringent single measure ICC as the parameter for interrater reliability (Shrout \& Fleiss, 1979). Internal consistency of the Dutch version of the revised BEST-Index and the derived factors was excellent. Item homogeneity was acceptable for the revised BEST-Index total score and the derived factors. Similar values for internal consistency have previously been reported (Woods et al., 1999).

Good convergent validity of the Dutch version of the BEST-Index with the HCR20 was found. As expected, the scores on the revised BEST-Index total score and the derived factors were more strongly associated with the changeable (i.e., dynamic) violence risk factors of the HCR-20, compared to the historical (i.e. static) factors of the HCR-20. Thus, it seems that the BEST-index is especially valuable as a measure of dynamic risk factors. Values for convergent validity of the BEST-index were in the same range as previously reported by Ross et al. (2008). Further, moderate to large correlation coefficients were found between the revised BESTIndex factors with diverse aggressive behaviors during institutional treatment, supporting its predictive validity. Moreover, in this study, the predictive results for the revised BEST-Index were comparable to, if not higher than, the predictive results for the HCR-20. Especially, the derived BEST-Index factor Interpersonal Hostility shows stronger associations with the diverse inpatient aggressive behaviors than the HCR-20. Note that the assessments with the BEST-index and the HCR-20 were independent of each other and both preceded the measurement of the START outcome variables.

The findings of the current study should be considered with several limitations in mind. The generalizability of the findings is limited to inpatient male forensic psychiatric patients. The study should be replicated in different samples (e.g., female forensic psychiatric patients) to assess the robustness of our findings. In addition, the sample size $(N=291)$ was somewhat small for the CFA and PCA analyses in relation to the number of BEST-items $(N=70)$. However, recommendations concerning the number of subjects required per item of the instrument vary widely within the literature: from 5 subjects per variable (Gorsuch, 1983) to 10 subjects per variable (Nunnally, 1967). MacCallum, Widaman, Zhang, and Hong (1999) demonstrated that the recommended sample size is influenced by the extent to which factors are overdetermined and the level of communalities of the measured variables. In our sample the average communality was .55, and 7 to 23 variables represented each factor. According to MacCallum et al. (1999), these are moderate to good conditions for a factor analysis and one can obtain good recovery of factors with samples of at least 200 . However, further studies should be performed using the BEST-Index with larger samples to test whether the current factor structure can be replicated.

Despite these limitations, the findings of the present study suggest that the Dutch version of the BEST-Index can be used reliably in Dutch forensic psychiatric settings, with four factorial domains that have apparent theoretical and clinical relevance, as demonstrated by their relationship with violence risk level and institutional aggressive behavior. Modifying the Dutch version of the BEST-Index was not central to this study, but our findings indicate that future research should further examine the suitability of a number of the BEST-Index items. This study also 
shows some of the strengths of the BEST-Index as a clinical instrument for the assessment of potentially risk-relevant behaviors among forensic psychiatric patients. An instrument that (a) has a broad content coverage relevant to the treatment of forensic psychiatric patients, (b) is related to future violence risk as measured with the HCR-20 and to institutional aggressive behavior, (c) can be reliably rated by paraprofessionals who have received only a half-day of training and in a relatively brief amount of time (1-2 hours) clearly has significant advantages in terms of cost-effectiveness and ease of implementation. More importantly, this study also shows the possibility of informing the structured assessment of future violence risk with measures such as the HCR-20, with standardized ratings from nursing staff on changes in risk-relevant behaviors during forensic treatment. Finally, future research is needed that examines the predictive validity of the BESTIndex for future (violent) offending in the community, as well as studies that assess the sensitivity of the BEST-Index for detecting change in future violence risk.

\section{Footnotes}

${ }^{1}$ The internal consistencies for the scales of the BEST-Index original version were $\alpha$ $=.96$ for the total scale, $\alpha=.79$ for the Risk subscale, $\alpha=.95$ for the Insight subscale and $\alpha=.96$ for the Communication and Social Skills subscale. The item homogeneity of the original version was .26 for the total scale, and .18 for the Risk subscale, .51 for the Insight subscale and .42 for the Communication and Social Skills subscale.

2 The interrater reliability for the scales of the BEST-Index original version were .72 for the total scale, .71 for the Risk subscale, .66 for the Insight subscale and .67 for the Communication and Social skills subscale.

3 To determine whether the results of the predictive analyses involving START outcome data would differ if we used a more appropriate predictive model given the nature of the distribution, we also analyzed these data using a negative binomial regression model. The resulting coefficients for both the revised BEST-Index and the HCR-20 showed the same magnitude of the reported effects as the Pearson correlation analyses. 
36 | CHAPTER 2 


\section{CHAPTER 3}

Early Maladaptive Schemas in Relation to Facets of Psychopathy \& Institutional Violence in Offenders with Personality Disorders

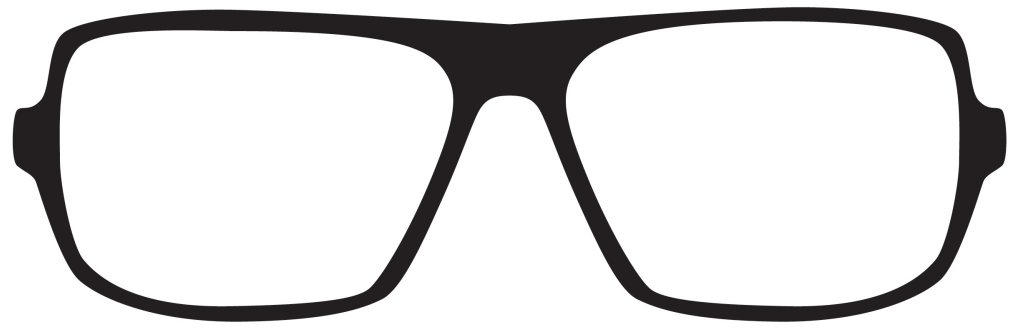




\section{SUMMARY}

Current knowledge suggests that the psychopathy construct is multifaceted in nature, and reflects different underlying pathological mechanisms, including neurobiological dysfunction and maladaptive cognitions. Whilst many contemporary studies focus on neurobiological aspects of psychopathy, few have addressed the maladaptive cognitions. In this study, we examined facets of Hare's psychopathy construct in terms of their associations with maladaptive cognitions, as defined by Young's cognitive theory of Early Maladaptive Schemas (EMS). Personality disordered offenders $(N=124)$ were assessed with the PCL-R and the Young Schema Questionnaire. The PCL-R Lifestyle and Antisocial Facets were significantly related to EMS Mistrust/Abuse and Insufficient Self-Control, consistent with our hypotheses, and were significantly, but negatively, related to EMS Subjugation. Also as hypothesized, EMS showed no associations with the PCL-R Affective and Interpersonal facets. Contrary to our expectation, EMS did not predict institutional violence. Our findings suggest that schemas relating to mistrust, inadequate selfcontrol/low frustration tolerance, and autonomy/dominance, play a role in the impulsive lifestyle and antisocial behavior features of psychopathy. Treatments that focus on ameliorating these schemas may lead to better outcomes in psychopathic offenders.

\section{INTRODUCTION}

Psychopathy affects about 0.6 - 1.2\% of the general population (Coid, Yang, Ullrich, Roberts, \& Hare, 2009a; Neumann \& Hare, 2008) and prevalence rates have been reported consisting of $7.7 \%$ for correctional settings in England and Wales (Coid et al., $2009 \mathrm{~b}), 15 \%$ to $25 \%$ of the North-American forensic and correctional samples (Hare, 2003), and $24 \%$ of the Dutch forensic psychiatric samples (Hildebrand, Hesper, Spreen, \& Nijman, 2005). According to Hare (1991), psychopathy can be defined as a constellation of interpersonal, affective, lifestyle, and antisocial characteristics. The personality features of psychopathy, such as lack of remorse, sensation seeking, and pathological lying are associated with a socially deviant lifestyle that includes criminal and violent behavior. Hare's work on psychopathy resulted in a 20-item clinical rating scale to assess the core characteristics of psychopathy. The Psychopathy Checklist-Revised (PCL-R; Hare, 1991, 2003) has become a diagnostic standard for assessing psychopathy (Lynam \& Gudonis, 2005). Recent studies, using structural equation modeling, suggest that the PCL-R can be broken down into four facets: An arrogant and manipulative interpersonal style (Interpersonal facet), deficient affective experience (Affective facet), impulsive and irresponsible behavior (Impulsive facet), and specific antisocial behaviors (Antisocial facet; Hare, 2003; Neumann, Hare, \& Newman, 2007). An alternative, hierarchical three-factor structure has been proposed in which there is less emphasis on criminal behavior, using 13 PCL-R items divided over three facets: Arrogant and Deceitful Interpersonal Style, Deficient Affective Experience, and Impulsive and Irresponsible Behavioral Style (Cooke \& Michie, 2001). The main difference between the four- and three-facets models is the omission of the items 
related to criminal behavior (i.e., the Antisocial facet), as scholars hold different views on whether criminality is an integral part or merely a consequence of psychopathy (Hare \& Neumann, 2010; Skeem \& Cooke, 2010). The PCL-R has shown to be a good predictor of general and violent recidivism (Hemphill, Hare, \& Wong, 1998; Leistico, Salekin, DeCoster, \& Rogers, 2008).

Previous research suggests differential relationships of the PCL-R facets with external criterion-related domains. For example, the Interpersonal facet of the PCL-R has been associated with instrumental violence, social dominance, low stress reactivity, and higher adaptive functioning (Hall, Benning, \& Patrick, 2004; Walsh, Swogger, \& Kosson, 2009). Deficient affective experience found in psychopaths, as reflected by the Affective facet, has been associated with neurobiological deficits in the limbic and other brain regions (Blair, 2003; Kiehl et al., 2001; Raine, Lencz, Bihrle, LaCasse, \& Colletti, 2000). The Lifestyle facet, including reactive aggression, has been shown to be associated with early traumatic experiences (Lang, af Klinteberg, \& Alm, 2002; Poythress, Skeem, \& Lilienfeld, 2006), serotonin dysfunction (Dolan \& Anderson, 2003), and suicidal behavior (Douglas et al., 2008; Verona, Patrick, \& Joiner, 2001). The Antisocial facet has been more strongly associated with crime-related behavioral outcome such as institutional violence, and general and violent recidivism than the other facets (Kennealy, Skeem, Walters, \& Camp, 2010; Walters, Knight, Grann, \& Dahle, 2008; Walters \& Heilbrun, 2010).

Although most contemporary research suggests that dysfunctions in specific brain regions is central to psychopathic traits in offenders as measured with the PCL-R (e.g., Kiehl et al., 2001; Tiihonen et al., 2008; see Yang \& Raine, 2009), little is known about the contribution of early developmental risk factors, such as childhood trauma, to psychopathic traits (Daversa, 2010). There is evidence from longitudinal studies in community samples that adverse experiences in childhood are associated with psychopathic traits in adulthood (Lang et al., 2002; Farrington, 2006; Weiler \& Widom, 1996). For example, in a 20-year follow-up study, Weiler and Widom (1996) compared 652 persons who were a documented victim of abuse and/or neglect during childhood (before the age of 11 years) with a matched control group ( $n=489$ ) and found that the victims of childhood abuse and/or neglect had significantly higher PCL-R scores than the persons in the matched control group. Similarly, Lang and colleagues (2002) found that victimization in childhood was related to violence and high psychopathy scores in adulthood in a Scandinavian community sample $(N=199)$. Also, results from the Cambridge Study in Delinquent Development (Farrington, 2006), a 40-year prospective study following 411 males on the development of offending and antisocial behavior from age 8 to 48, suggest that adverse experiences (such as poor parental supervision, emotional and physical neglect) at age 8-10 predicted high scores on the PCL Screening Version (PCL-SV; Hart, Cox, \& Hare, 1995) in adulthood. There is also evidence from crosssectional studies in offender samples that childhood trauma is associated with higher PCL-R scores (Graham, Kimonis, Wasserman, \& Kline, 2012; Koivisto \& Haapasalo, 1996; Marshall \& Cooke, 1999; Poythress et al., 2006; WeizmannHenelius et al., 2010). For example, in a sample of 223 convicted sex offenders, Graham and colleagues (2012) found that childhood physical abuse and/or neglect based on archived evaluation reports was associated with a higher score on the PCL-R Antisocial facet. Similar results linking childhood abuse to psychopathic 
features were found in a study of Poythress and colleagues (2006). In a sample of 615 prisoners and subjects court ordered to residential drug treatment, selfreported childhood abuse was significantly associated with both the PCL-R Impulsive Lifestyle facet and the Antisocial facet.

Although Antisocial Personality Disorder (APD) and psychopathy are distinct disorders (Ogloff, 2006), APD shares overlap with the behavioral characteristics (i.e., Lifestyle and Antisocial facets) of psychopathy (Hare, 2003; Ogloff, 2006). For example, a diagnosis of APD is strongly correlated with PCL-R behavioral characteristics in North-American forensic psychiatric samples $(r=.59$; Hart \& Hare, 1989), as well as in Dutch forensic psychiatric samples ( $r=.65$; Hildebrand $\&$ de Ruiter, 2004). The literature on the relationship between early adverse experiences and APD may be relevant for the PCL-R Lifestyle and Antisocial facets. For example, early adverse experiences have been demonstrated to be a longitudinal risk factor for APD (Luntz \& Widom, 1994) and offending in adolescence and/or adulthood (e.g., Lansford et al., 2007; Maxfield \& Widom, 1996; Smith \& Thornberry, 1995; Thornberry, Ireland, \& Smith, 2001). Also, Iongitudinal studies examining the interaction between genetic and environmental influences have found that childhood abuse is a strong risk factor for APD symptoms and antisocial behavior in adulthood (e.g., Caspi et al., 2002; Huizinga et al., 2006), and that this relationship was moderated but not limited by genetic traits predisposing individuals towards impulsive, aggressive, and violence-related behavioral problems (Caspi et al., 2002). These findings suggest that early adverse experiences are a risk factor for developing APD symptoms and antisocial behavior in adulthood, for both individuals with and without the genetic traits towards such behavior.

Most current interventions aimed at reducing aggressive behavior and relapse prevention are based on cognitive-behavioral theories of psychopathology (Hollin \& Palmer, 2006). Deviant cognitions play an important role in causing and maintaining violent and criminal behavior. Beck, Freeman, and colleagues (1990) have hypothesized that maladaptive cognitions develop in early childhood under the influences of adverse life experiences and play a central role in personality disorders, including those that are prevalent in forensic settings, such as Antisocial, Borderline, and Narcissistic Personality Disorders. According to Beck and colleagues (1990) highly antisocial individuals are more likely to manifest maladaptive cognitions that emphasize immediate personal satisfaction while minimizing future consequences, and they are more likely to be highly distrusting and have a hostile view of the world in terms in terms of predators and prey. However, thus far, there have been few attempts in the scientific literature to apply or test cognitive models of psychopathy.

Young and colleagues (Young, Klosko, \& Weishaar, 2003), building on the work of Beck et al. (1990), developed a cognitive theory for personality disorders based on early maladaptive schemas (EMS; see Table 1). According to Young et al. (2003), EMS are chronic, maladaptive patterns of cognition and affect that originate in adverse childhood experiences and early temperament, that, in combination with maladaptive coping responses, influence interpersonal interactions and guide behavior. Young et al. (2003) hypothesized that EMS lie at the core of personality disorders and externalizing behaviors are primarily maladaptive coping responses to EMS. The theory on EMS may also be relevant for personality disordered offenders 
and to other severe personality disturbances, such as psychopathy (Bernstein, Arntz, \& de Vos, 2007; Bernstein et al., 2012).

\section{TABLE 1}

Description of the early maladaptive schemas and domains proposed by Young (1994)

Schema domain and scales

Description

\section{Disconnection/Rejection}

Emotional Deprivation

Abandonment

Mistrust/Abuse

Social Isolation

Defectiveness/Shame

Impaired Autonomy/Performance

Failure to Achieve

Dependence/Incompetence

Vulnerability

Enmeshment

\section{Impaired Limits}

Entitlement

Insufficient Self-Control

\section{Other-Directedness}

Subjugation

Self-sacrifice

\section{Over-Vigilance/Inhibition}

Emotional Inhibition

Unrelenting Standards

Belief that one's emotional needs will not adequately be met by others

Expectation of being abandoned by significant others

Expectation of being abused, mistreated or cheated by others

Belief of being different for other people, being isolated from the rest.

Belief of being defective or inferior in important aspects

Belief of being fundamentally inadequate and will inevitably fail

Belief of being incompetent in handling daily responsibilities without help

Belief that emotional or medical catastrophe will be imminent

Belief that to survive, excessive emotional involvements are necessary

Expectation that one is superior, and can act without regard for others

Pervasive difficulty to exercise self-control or frustration tolerance

Belief that one must be excessive compliant to avoid anger or abandonment

Belief that one must meet the needs of others, at expense of their own needs

Belief that one must inhibit spontaneous action, feelings or communications

Belief that one must meet very high standards of behavior and performance 
Young et al. (2003) stated no specific hypotheses about EMS that may be prevalent in APD or psychopathy. However, following Beck and colleagues (1990) theory on maladaptive cognitions in APD, several authors suggested specific EMS incorporated in the Young Schema Questionnaire (YSQ; Young \& Brown, 1994) such as Mistrust/Abuse, Emotional Inhibition, Entitlement, and Insufficient Self-Control that may be relevant for aggressive, antisocial behavior (e.g., Ball \& Cecero, 2001; Tremblay \& Dozois, 2009). To our knowledge, there has been no study investigating EMS in offenders high on psychopathy and/or violent offenders with personality disorders. However, there has been one study investigating EMS in a sample of DSM-IV cluster B (opiate dependent) personality disordered patients; Ball and Cecero (2001) found support for the relationship between Mistrust/Abuse, Emotional Inhibition and APD symptoms. Also, some support has been found in non-clinical samples for the relationship between the EMS and aggression. For example, one study found an association between Entitlement and self-reported aggressive behavior in a large non-clinical adolescent sample $(N=974$; Calvete \& Orue, 2011), and two other studies in large undergraduate samples $(N=407$ 848) found significant relationships between self-reported aggression and the EMS Mistrust/Abuse, Insufficient Self-Control, and Entitlement (Calvete, Estevez, López de Arroyabe, \& Ruiz, 2005; Tremblay \& Dozois, 2009).

Overall, this body of literature suggests that early adverse experiences may be a potentially relevant etiological factor in the development of psychopathy, particularly in its antisocial and impulsive lifestyle components, and thus it is important to examine whether the cognitive sequelae of early adverse experiences, in terms of maladaptive schemas (Beck et al., 1990; Young et al., 2003), are related to psychopathic traits in adulthood. More specifically, the EMS Mistrust/Abuse, Emotional Inhibition, Entitlement, and Insufficient Self-Control, are hypothesized to underlie antisocial behavior in adulthood (Beck et al., 1990). Therefore, in the present study the following hypotheses were derived: (1) in light of previous findings (Kennealy et al., 2010; Walters et al., 2008; Walters \& Heilbrun, 2010), as well as theoretical considerations (Beck et al., 1990; Young et al., 2003), we hypothesized that EMS Mistrust/Abuse, Emotional Inhibition, Entitlement, and Insufficient Self-Control predicted the PCL-R Impulsive Lifestyle and the Antisocial Behavior facets. In contrast, (2) no relationship is expected between EMS and the PCL-R Affective facet. There is evidence that the PCL-R Affective facet has a strong neurobiological basis (e.g., Raine et al., 2000), and that callousunemotional traits in children have a strong genetic basis (Viding, Blair, Moffitt, \& Plomin, 2005). Given the neurobiological basis of psychopaths' affective deficits, we expected that this facet would be essentially independent of the EMS incorporated in the YSQ. Also, (3) we hypothesized that the PCL-R Interpersonal facet would show little or no correlation with EMS because the PCL-R Interpersonal facet reflects deviant coping responses or maladaptive social functioning associated with social dominance and low stress reactivity (Hall et al., 2004), rather than maladaptive cognitive schemas. Finally, (4) we hypothesized that EMS Mistrust/Abuse, Emotional Inhibition, Entitlement, and Insufficient Self-Control, will have incremental validity above the PCL-R Impulsive Lifestyle facet and the PCL-R Antisocial facet in predicting institutional violence in a sample of personality disordered offenders over a follow-period of two years. 


\section{METHOD}

\section{Sample}

Subjects were 124 male offenders, involuntarily admitted to a forensic psychiatric hospital under the Dutch judicial measure of "TerbeschikkingStelling" imposed on offenders who have committed serious offences, carrying a punishment of at least 4 years imprisonment, and who suffer from a mental disorder according to DSM-IV. Subjects met diagnostic criteria for one or more DSM-IV personality disorder(s). Mean age of the sample was 37.5 years $(S D=8.9$, range $=22$ to 58 ), and $77.3 \%$ of the patients were of Dutch origin. One percent of the sample was of Eastern European origin, and $21.7 \%$ were of non-European origin (e.g., Turkey, Morocco, Brazil). In the sample, $43.5 \%$ of the patients were convicted for (attempted) homicide, $18.5 \%$ for sexual offenses, $28.2 \%$ for violent theft, robbery, and assault, and $9.7 \%$ for arson. DSM-IV Axis I and Axis II diagnoses were extracted from the patient files, which were based on clinical and/or semi-structured interview diagnoses made by psychiatrists and clinical psychologists. The most prevalent personality disorder (PD) in the sample was a mixed PD or PD Not Otherwise Specified (56.5\%), followed by Antisocial PD (32.2\%), Borderline PD (16.9\%), Narcissistic PD (9.7\%) and Paranoid PD (2.4\%). Other PD's were not present. Regarding Axis I diagnosis, 78.2\% met criteria for at least one substance use disorder, $18.5 \%$ met criteria for attention deficit and disruptive behavior disorders, $12.9 \%$ for mood disorders, $9.7 \%$ for impulse control disorders, and $7.3 \%$ for other DSM-IV Axis I disorder categories.

\section{Materials}

Young Schema Questionnaire. The Young Schema Questionnaire (YSQ; Young \& Brown, 1994; Dutch version: Sterk \& Rijkeboer, 1997; Young \& Pijnaker, 1999) is a self-report instrument designed to assess early maladaptive schemas. It consists of 205 items, each rated from 1 (completely untrue) to 6 (describes me perfectly). Subjects have to endorse how well a statement describes them (e.g., I subscribe to the belief: "Control or be controlled"). The items are divided among 15 subscales in five broad domains (see Table 1). The first domain is labeled Disconnection/Rejection and consists of the scales: Emotional Deprivation, Abandonment, Mistrust/Abuse, Social Isolation, Defectiveness/Shame. The second domain is called Impaired Autonomy/Performance and contains the scales: Failure to Achieve, Dependence/Incompetence, Vulnerability to Harm, Enmeshment. The third domain is labeled Impaired Limits and consists of the scales: Entitlement and Insufficient Self-Control. The fourth domain labeled Other-Directedness, consists of the scales: Subjugation and Self-Sacrifice. The last domain is labeled Overvigilance/Inhibition, which consists of the scales: Emotional Inhibition and Unrelenting Standards. The overall score on each subscale or domain is obtained by calculating the mean of the items in that scale or domain. For all subscales and domains, a higher score reflects a more maladaptive and pervasive core belief. The 15 EMS emerged as primary factors in a study by Schmidt, Joiner, Young, and Telch (1995) in a student sample $(N=1129)$ and a clinical sample $(N=187)$, and similar factor structure results were obtained by Lee, Taylor, and Dunn (1999) in an 
Australian clinical sample $(N=433)$. Schmidt and colleagues (1995) also demonstrated that the YSQ primary factors had adequate test-retest coefficients (range of $r=.50-.82$ ) and adequate internal consistency (range of $\alpha=.83-.96$ ). Research on the Dutch version of the YSQ in a combined sample of clinical and non-clinical populations shows that the Dutch version has adequate reliability, including test-retest (range of $r=.68-.86$; Rijkeboer, van den Bergh, \& van den Bout, 2005) and internal consistency reliability (range of $\alpha=.76-.95$; Rijkeboer \& van den Bergh, 2006). Furthermore, the Dutch YSQ showed good discriminating power, in terms of predicting whether subjects were clinical or non-clinical $(88.3 \%$ correctly classified; Rijkeboer et al., 2005).

In this study, we combined two separately translated versions of the Dutch YSQ, both of which had been used at different times for the clinical assessment of patients at the hospital. The first version, which was introduced in the center in 2001, was published in an appendix to the Dutch version of "Schema Therapy: $A$ Practitioner's Guide" (Young \& Pijnaker, 1999). The second version was separately translated and validated by Sterk and Rijkeboer (1997). It was adopted by the hospital in 2005 because of its strong reliability and validity in other Dutch samples (Rijkeboer \& van den Bergh, 2006; Rijkeboer et al., 2005). The two versions differ in semantic sentence structure and item dispersion (e.g., random dispersion versus ordered by schema domain). In our sample, we found no differences between the two versions in internal consistency (version 1: range of $\alpha=.76-.96$, average $\alpha=$ .88 and version 2 : range of $\alpha=.81-.97$, average $\alpha=.89$ ) and item homogeneity (version 1: range of $r=.21-.58$, average $r=.35$ and version 2: range of $r=.29$ .58 , average of $r=.37$ ). Thus, because their psychometric properties appeared to be comparable, we felt justified to combine the data of the two questionnaire versions for the present study purposes.

Psychopathy Checklist-Revised. The Psychopathy Checklist - Revised (PCL-R; Hare, 1991, 2003; Dutch version: Vertommen, Verheul, de Ruiter, \& Hildebrand, 2002 ) is a 20-item clinical construct rating scale designed to assess psychopathy in forensic populations. Each item is rated on a scale from 0 to $2(0=$ does not apply, 1 = applies to some extent, 2 = definitely applies). The PCL-R provides a dimensional total score between 0 and 40 indicating the degree to which the individual matches the prototypical psychopath. In this study, we report results based on the PCL-R four-facet model (Hare, 2003) given its relationship with antisocial and violent outcome (Kennealy et al., 2010; Walters et al., 2008; Walters \& Heilbrun, 2010). The PCL-R four facet-model consists of the alternative threefactor model (Cooke \& Michie, 2001) and the Antisocial facet as a fourth facet.

Institutional violence. In this study, a modified version of the Overt Aggression Scale (OAS; Yudofsky, Silver, Jackson, Endicott, \& Williams, 1986) for use in forensic clinical practice was used (Nicholls, Brink, Desmarais, Webster, \& Martin, 2006) for measuring institutional violence. The modified version of the OAS expands on the original four categories in the OAS (i.e., verbal aggression, physical aggression against objects, physical aggression against self, and physical aggression against other people) by adding several other categories that may be relevant for forensic patients, such as self-neglect, substance abuse, sexual aggression, unauthorized leave, and suicidal behavior (Nicholls et al., 2006). Following the categorization of Hildebrand, de Ruiter and Nijman (2004) of 
institutional violence in a comparable sample, the following dimensions from the modified version were summed to obtain a score for institutional violence: (1) verbal aggression; (2) physical aggression; and (3) violations of hospital rules (i.e., unauthorized leave and substance abuse). Prior research showed that the modified version of the OAS can be reliably coded (intraclass correlation coefficient [ICC] = .70; Nicholls et al., 2006). In the sample, $88.7 \%$ has demonstrated acts of institutional violence during the follow-up period with an average of 12.0 acts (SD = 15.1) per patient.

\section{Procedure}

All patients admitted to the hospital during the period between March 1, 2000 and February 2, 2009 were initially screened for eligibility in this study. Initially, 193 patients were evaluated for participation, which included the presence of at least one DSM-IV personality disorder. Sixty-nine patients with borderline intellectual functioning, psychotic-, paraphilic-, and autism spectrum disorders were excluded. The resulting sample consists of 124 personality disordered offenders and represents $64.3 \%$ of the total sample. Patients completed the YSQ at admission, as part of the hospital intake procedure. PCL-R assessments were performed during the course of treatment $(M=1.9$ years after admission, $S D=1.8)$ by two certified and independent raters. In $48.4 \%$ of the cases, PCL-R assessments were based on an interview combined with information from the hospital files containing criminal records, mental health reports, violence risk assessments, medical information, treatment progress reports, and reports from psychiatric nurses, social workers, work- and education supervisors. The PCL-R assessments in the other cases (51.6\%) were based on the file-only procedure. For 112 cases, data on interrater reliability for the PCL-R total score were available revealing an average measure ICC of .80.

Official registration of institutional misconduct was used to code aggressive behaviors on the modified OAS scale. Possible antecedents, motive, and a detailed description of the observed misconduct were registered by psychiatric nurses at each occurrence. To classify this misconduct in terms of type and severity, all misconducts were re-coded by a rater (a masters-level research assistant) with the English version of the modified OAS scale (Nicholls et al., 2006). To ensure a prospective design, all misconducts that occurred prior to the assessment of the YSQ and/or PCL-R were eliminated from the analyses. Prior research in a larger sample showed that the modified version of the OAS can be reliably coded in the study setting (ICC = .83; Chakhssi, de Ruiter, \& Bernstein, 2010a). Written informed consent was obtained from the patients prior to the assessments and approval for the study was obtained from the institutional research review committee.

\section{Statistical analysis}

Internal consistency was examined using Cronbach's alpha for the YSQ domains as well as for the YSQ scales (EMS). Mean inter-item correlations were used as a measure of item homogeneity. Zero-order correlations using Pearson's $r$ were calculated to examine the relationship between PCL-R facets and EMS. Type I error inflation was controlled by using the Finner correction for multiple comparisons 
(Finner, 1993). The Finner correction is an adjustment of the Bonferroni correction and uses a sequential rejective procedure that is based on the ordered $p$-values of the individual comparisons. The Finner correction has been demonstrated to be less conservative but more powerful than the traditional Bonferroni correction and has a lower Type II error rate (Finner \& Strassburger, 2002). Given the paucity of empirical data on EMS in offenders with psychopathic traits, we corrected for multiple comparisons per PCL-R facet. This way, our study may detect significant associations between other EMS and PCL-R facets that may otherwise have gone unnoticed and may provide promising leads for future studies.

Multiple stepwise linear regression analyses were used to examine the relative contributions of the hypothesized EMS to the PCL-R facet scores. Also, multiple stepwise linear regression analyses were used to examine the association between PCL-R facets and institutional violence, and the association between EMS and institutional violence. Hierarchical regression analyses were then used to examine the incremental validity of the hypothesized EMS above PCL-R facets in predicting institutional violence. All statistical analyses were performed using the Statistical Package for the Social Sciences, version 13.0 (SPSS Inc., 2005).

\section{RESULTS}

In this sample, the mean PCL-R score was $22.47(S D=6.61)$. Fourteen patients had a PCL-score $\geq 30$ and 44 patients had a PCL-score $\geq 25$. For the PCL-R facets as defined by Hare (2003), the mean score for the Interpersonal facet was 3.74 $(S D=2.08), 5.79(S D=1.91)$ for the Affective facet, $5.41(S D=2.34)$ for the Impulsive lifestyle facet, and $5.56(S D=2.64)$ for the Antisocial facet. For descriptive purposes, means, standard deviations, internal consistency (Cronbach's alpha), and mean inter-item correlation for the YSQ higher-order domains and scales are provided in Table 2. Overall, the internal consistency was high, ranging from $\alpha=$ .82 for the YSQ Subjugation scale to $\alpha=.97$ for the higher-order YSQ schema domain Disconnection/Rejection. The mean inter-item correlations per scale were also satisfactory and ranged from .26 for the YSQ Other-Directedness domain to .58 for the YSQ Emotional Deprivation scale.

Significant zero-order correlations were found for the PCL-R Impulsive Lifestyle and Antisocial facets with EMS (see Table 3) after Finner correction for multiple comparisons. Inspection of Table 3 shows that for the PCL-R Impulsive Lifestyle facet, 4 out of 15 possible correlations with the EMS were significant. The PCL-R Antisocial facet was significantly associated with the Mistrust/Abuse schema. The PCL-R Interpersonal facet and Affective facet showed no significant correlations with EMS.

For both the PCL-R Impulsive Lifestyle facet and the PCL-R Antisocial facet, stepwise multiple regression analysis was performed with all the EMS entered as predictors, to examine if the hypothesized EMS would emerge as independent predictors.

For the PCL-R Impulsive Lifestyle facet, the regression analysis revealed that three EMS explained $19.2 \%$ ( $r^{2}$ adjusted) of the variance $[F(3,120)=10.77, p<$ .01]. It was found that Insufficient Self-Control significantly predicted the PCL-R Impulsive Lifestyle facet $[\beta=.45, t(120)=3.64, p<.01]$, as did Subjugation $[\beta=-$ 
$.39, t(120)=-3.50, p<.01]$ and Mistrust/Abuse $[\beta=.24, t(120)=2.17, p=$ .032]. In other words, high Insufficient Self-Control, high Mistrust/Abuse, and low levels of Subjugation predicted higher PCL-R Impulsive facet scores.

\section{TABLE 2}

Mean scores, standard deviations, item homogeneity, and internal consistency of the YSQ domains in a sample of personality disordered offenders $(n=124)$

\begin{tabular}{llllll}
\hline Schema domain and scales & Nit & $M$ & SD & $\alpha$ & MIC \\
\hline Disconnection/Rejection & 69 & 2.02 & 0.81 & .97 & .31 \\
Emotional Deprivation & 9 & 2.46 & 1.33 & .92 & .58 \\
Abandonment & 18 & 1.99 & 0.94 & .92 & .39 \\
Mistrust/Abuse & 17 & 2.24 & 1.01 & .92 & .40 \\
Social Isolation & 10 & 1.84 & 0.90 & .85 & .40 \\
Defectiveness/Shame & 15 & 1.59 & 0.76 & .90 & .38 \\
Impaired Autonomy/Performance & 49 & 1.70 & 0.71 & .96 & .34 \\
Failure to Achieve & 9 & 1.86 & 0.99 & .89 & .50 \\
Dependence/Incompetence & 15 & 1.70 & 0.76 & .90 & .40 \\
Vulnerability & 14 & 1.69 & 0.73 & .86 & .33 \\
Enmeshment & 11 & 1.58 & 0.69 & .84 & .32 \\
Impaired Limits & 26 & 2.02 & 0.74 & .91 & .27 \\
Entitlement & 11 & 1.98 & 0.80 & .83 & .31 \\
Insufficient Self-Control & 15 & 2.06 & 0.85 & .88 & .32 \\
Other-Directedness & 27 & 2.20 & 0.74 & .89 & .26 \\
Subjugation & 10 & 1.79 & 0.77 & .82 & .36 \\
Self-sacrifice & 17 & 2.62 & 0.87 & .86 & .27 \\
Over-Vigilance/Inhibition & 25 & 2.19 & 0.83 & .90 & .27 \\
Emotional Inhibition & 9 & 2.10 & 1.01 & .85 & .39 \\
Unrelenting Standards & 16 & 2.28 & 0.87 & .86 & .38 \\
\hline Note. Nit N Number of & & & & & .36 \\
& & & & &
\end{tabular}

Note. Nit = Number of items; $a$ = internal consistency; MIC = mean interitem correlation.

For the PCL-R Antisocial facet, the regression analysis revealed Mistrust/Abuse $[\beta=.32, t(120)=2.85, p<.01]$, Subjugation $[\beta=-.47, t(120)=-4.12, p<.01]$ and Insufficient Self-Control $[\beta=.33, t(120)=2.65, p<.01]$, significantly predicted the PCL-R Antisocial Lifestyle facet, accounting for $17.3 \%$ ( $r^{2}$ adjusted) of the variance $[F(3,120)=9.59, p<.01]$. Thus, high Insufficient Self-Control, high Mistrust/Abuse, and low levels of Subjugation predicted higher PCL-R Antisocial facet scores.

To examine the assumption that the hypothesized EMS would not be related to the PCL-R Affective and Interpersonal facets, additional multiple regression analyses were performed using the same procedure as above. These analyses 
revealed no significant overall models (all $p$ 's $>.05$ ) and no significant predictors (all $p$ 's > .05) for the PCL-R Interpersonal and Affective facets.

Next, we performed a multiple regression analysis with all the PCL-R facets entered as predictors and institutional violence as the dependent variable. The results showed that the only the PCL-R Antisocial facet emerged as a significant predictor $[\beta=.20, t(119)=2.05, p=.043]$, but not the other PCL-R facets (all $\mathbb{W L}$ $p$ 's $>.05)$, explaining $12.2 \%$ of the variance ( $r^{2}$ adjusted) in institutional violence [ $F$ $(4,119)=5.29, p<.001]$. Also, a stepwise multiple regression analysis was performed with all EMS entered as predictors and institutional violence as the dependent variable. The results showed that the overall model was not significant and no EMS emerged as a significant predictor. Given these findings, additional analyses to examine the incremental validity of the EMS above the PCL-R Impulsive Lifestyle and Antisocial facet in predicting institutional violence were not performed.

\section{TABLE 3}

Zero order correlations between PCL-R facets and Early Maladaptive Schemas

\begin{tabular}{|c|c|c|c|c|}
\hline \multirow{3}{*}{ Schema scales } & \multicolumn{4}{|c|}{ PCL-R facets } \\
\hline & \multirow[t]{2}{*}{ Interpersonal } & \multirow[t]{2}{*}{ Affective } & Impulsive & Antisocial \\
\hline & & & \multicolumn{2}{|l|}{ Lifestyle } \\
\hline Emotional Deprivation & -.03 & -.08 & .17 & .08 \\
\hline Abandonment & -.08 & -.05 & .19 & .13 \\
\hline Mistrust/Abuse & .04 & -.02 & $.32 * *$ & $.28 *$ \\
\hline Social Isolation & -.03 & .00 & $.24^{*}$ & .06 \\
\hline Defectiveness/Shame & -.05 & -.09 & .16 & -.02 \\
\hline Failure to Achieve & -.10 & -.04 & .21 & -.03 \\
\hline Dependence/Incompetence & -.06 & .07 & $.22 *$ & .09 \\
\hline Vulnerability & .01 & .00 & .17 & .03 \\
\hline Enmeshment & -.01 & .07 & .18 & .04 \\
\hline Entitlement & .01 & -.15 & .20 & .16 \\
\hline Insufficient Self-Control & -.15 & -.02 & $.34 * *$ & .22 \\
\hline Subjugation & -.14 & -.14 & .04 & -.07 \\
\hline Self-sacrifice & .02 & -.07 & .03 & -.05 \\
\hline Emotional Inhibition & -.14 & -.13 & .13 & .04 \\
\hline Unrelenting Standards & .09 & .00 & .03 & .07 \\
\hline
\end{tabular}

Note. ${ }^{*} p<.05 ; * * p<.01$ (two-tailed), all $p$-values Finner corrected. 


\section{DISCUSSION}

This study examined the association between early maladaptive schemas, psychopathy facets, and institutional violence in an inpatient sample of personality disordered offenders. The findings show that early maladaptive schemas of Mistrust/Abuse, Insufficient Self-Control, and Subjugation were significantly related to the PCL-R Impulsive Lifestyle and Antisocial facets, consistent with Beck et al.'s (1990) hypotheses on maladaptive cognitions in highly antisocial individuals, and also in line with prior research that severity of APD symptoms is associated with early maladaptive schemas (Ball \& Cecero, 2001). These schemas refer to a high level of mistrust and a hostile view of the world in terms in terms of predators and prey (Mistrust/Abuse), to inadequate self-control and low frustration tolerance (Insufficient Self-Control), and to a strong need for autonomy and dominance (the inverse relationship with Subjugation). However, contrary to expectation, no relationship was found between the early maladaptive schema Entitlement and the PCL-R facets. This finding is consistent with previous research in personality disordered patients (Ball \& Cecero, 2001), but in contrast to findings from studies examining the association between Entitlement and self-reported aggressiveness in students (Calvete et al., 2005; Tremblay \& Dozois, 2009) and adolescents (Calvete \& Orue, 2011).

Interestingly, Subjugation emerged as a significant negative predictor in the regression analyses for both the Impulsive Lifestyle and the Antisocial facet, instead of the hypothesized EMS Emotional Inhibition, but was not significantly associated to these facets in the zero-order correlation analyses. This may be explained by the intercorrelation among the EMS (Rijkeboer \& van den Bergh, 2006) and, therefore, the partial information provided by the zero-order correlation was not sufficient to assess the unique contributions of the EMS. As suggested by Meehl (1945), theoretically relevant variables, including those variables that may be uncorrelated with the outcome variable in bivariate analyses, should not be dismissed for subsequent multivariate analyses, and may improve the predictive power of the regression model by partialling out invalid variance of the other predictors included in the model. Thus, in addition to the Finner corrected bivariate analyses, we used a multivariate approach to examine the unique associations of EMS with the PCL-R facets, while simultaneously controlling for all other EMS. Subjugation emerged as a significant predictor in both multivariate analyses for the PCL-R facets which suggest that this was not a "chance" finding and may have theoretical relevance (Paulhus, Robins, Trzesniewski, \& Tracy, 2004). An explanation could be that the Subjugation EMS refers to the feeling that one has to be excessively compliant in relationships, at the expense of one's own needs and emotions, in order to gain self-esteem and to avoid conflict (Young et al., 2003). The opposite, given the negative direction of the association, would probably be best described as individuals who do not want to be subjugated and may rebel against others who are subjugating or coercing them. In other words, they are egocentric (non-compliant in relationships), experience authority as restrictive and arbitrary, prone to override others' needs and wants resulting in frequent interpersonal conflicts. This description closely resembles the Antisocial, 
Interpersonal and/or Impulsive lifestyle features of psychopathy, and this could explain why it emerged as a significant predictor.

Consistent with our second hypothesis, our findings showed that the PCL-R Affective facet was independent of EMS. As suggested by Blair (2003), the dysfunctional neural structures, most likely the amygdala, implicated in the affective impairment of psychopathy, probably are a manifestation of genetic traits. Although Young (1994) hypothesized that EMS may develop partially under temperamental influences, no relationship was expected and found between the specific emotional deficits incorporated in the PCL-R Affective facet and EMS domains. As expected in our third hypothesis, we found no relationship between EMS domains and the PCL-R Interpersonal facet.

Our fourth hypothesis was not supported. Although previous research has shown that early adverse experiences are related to antisocial behavior in adulthood (e.g., Caspi et al., 2002; Weiler \& Widom, 1996), their cognitive sequelae, in terms of maladaptive schemas, were not related to institutional violence in our sample. As stated earlier, externalizing behaviors can be considered as maladaptive coping responses to EMS (Young et al., 2003). The maladaptive coping responses are not as stable as EMS and may change depending to the current life situation and events that activate EMS. Future research should examine whether EMS related maladaptive coping strategies are related to antisocial behavior, and/or if the emotional state-related manifestations of EMS, called schema modes, are related to antisocial behavior. Schema modes account for the rapid changes in mood and behavior observed in personality disordered patients when EMS are triggered in specific situations (Lobbestael \& Arntz, 2010). For example, antisocial patients may 'switch' between states of vigilance and aggression depending on the intensity and/or imminence of the anger triggering event (Lobbestael, Arntz, Cima, \& Chakhssi, 2009).

Our findings provide preliminary evidence that maladaptive cognitive schemas, presumably based in early childhood adversity, may play a role in psychopathy, particularly in its antisocial and impulsive lifestyle components. Psychopathic individuals have been described as highly distrusting, viewing the world in terms of predators and prey, victimizers and victims (Beck et al., 1990). Such cognitive schemas may arise under conditions where children are subjected to and/or witness to violence, are left unsupervised and unprotected, and experience instability and deprivation. Abuse and/or neglect experiences and their sequelae, in terms of cognitive schemas, do not play a role in most contemporary models of psychopathy, which tend to emphasize neuropsychological deficits in impulse control, affect recognition/regulation, and conditioned learning (e.g., Blair, 2003).

Our study has several limitations that should be taken into account. First, despite the clinical relevance of our findings, the effect sizes ranged from small to moderate. The largest effects were demonstrated for the Lifestyle facet, where 19\% of the variance was explained by the EMS. One explanation for the modest degree of variance explained could be that the variance in YSQ scores in our sample was limited in comparison to what has been found in non-forensic clinical samples (where the SD's ranged from .75 to 1.26; Rijkeboer et al., 2005), which may be a consequence of forensic patients' well-known tendency to minimize pathology on self-report inventories (Rogers, 1997). Thus, restriction of the range of YSQ scores 
may have led to an underestimation of the actual relationships between schemas and psychopathic features. Second, data from two versions of the YSQ were used in this study. Our preliminary analyses showed that the versions were psychometrically equivalent and nothing in our results suggested methodological ramifications. However, it would be preferable to use a single version of the YSQ in future studies. Third, we relied on self-report exclusively for the assessment of schemas. Although, the Dutch version of the YSQ is extensively validated (Rijkeboer et al., 2005), self-report is an explicit way of accessing cognitive schemas, and future studies should add experimental paradigms such as tests of implicit cognitions. Our findings on the association between psychopathic traits and maladaptive schemas should be interpreted cautiously until they are replicated in larger samples with both explicit and implicit measures. Fourth, we investigated the relationship of schemas and psychopathy in a forensic sample of personality disorder patients. The relationships between these variables might be different in a sample that also included non-offenders with psychopathic features. Finally, our design was correlational in nature and this limits the ability to ascertain whether the associations between EMS domains and psychopathy facets are causally related or merely due to shared developmental factors in childhood. No conclusions can be drawn about the causal role of cognitive schemas in psychopathy without further longitudinal and experimental studies that investigate the mechanisms responsible for these associations.

In conclusion, our findings suggest that maladaptive cognitive schemas may play a greater role in psychopathic traits than has been previously recognized. Future studies should further elucidate the cognitive schemas of personality disordered offenders with psychopathic traits, with the aim of informing more effective treatments for this challenging patient group. While genetically based neuropsychological deficits may not be as susceptible to change, cognitive schemas have proven to be modifiable in a broad range of mental disorders, including externalizing disorders such as substance abuse and borderline personality disorder (e.g., Ball, 2007; Farrell, Shaw, \& Webber, 2009; Giesen-Bloo et al., 2006; Nadort et al., 2009). For example, in a randomized clinical trial of borderline personality disordered outpatients $(N=86)$, three years of Schema Therapy (ST; Young et al., 2003) proved to be effective in reducing symptoms and improving general functioning (Giesen-Bloo et al., 2006). Almost half of the patients receiving ST were judged to be recovered from borderline personality disorder pathology, and $66 \%$ showed clinically significant improvement (Giesen-Bloo et al., 2006). Similar results have been reported in another randomized controlled trial (Farrell et al., 2009) and an open trial (Nadort et al., 2009), in which between 42\% and $94 \%$ of the patients were judged to have recovered from their borderline personality disorders symptoms or to have made clinically significant improvements.

Treatments which target maladaptive schemas may enable some personality disordered patients with psychopathic traits to develop more balanced and accurate appraisals of other persons' intentions, and to better cope with situations that would otherwise have triggered schema-related aggression. Treatments that focus on re-processing early traumas may help to lessen the severity of maladaptive schemas by decreasing the intensity of the memories, affects, and cognitions 
associated with them. Thus, our findings raise the possibility that cognitive, traumafocused treatments may enable some personality disordered patients with psychopathic features to achieve greater control over antisocial and impulsive behavior. As a logical next step, these potentially valuable avenues in the treatment of patients with psychopathic traits are now being explored in a randomized clinical trial comparing Schema Focused Treatment with treatment as usual in personality disordered offenders detained under the Dutch TBS-order (Bernstein et al., 2012). 


\section{CHAPTER 4}

Early Maladaptive Cognitive Schemas in Child Sexual Offenders compared to Sexual Offenders against Adults \& Nonsexual Violent Offenders

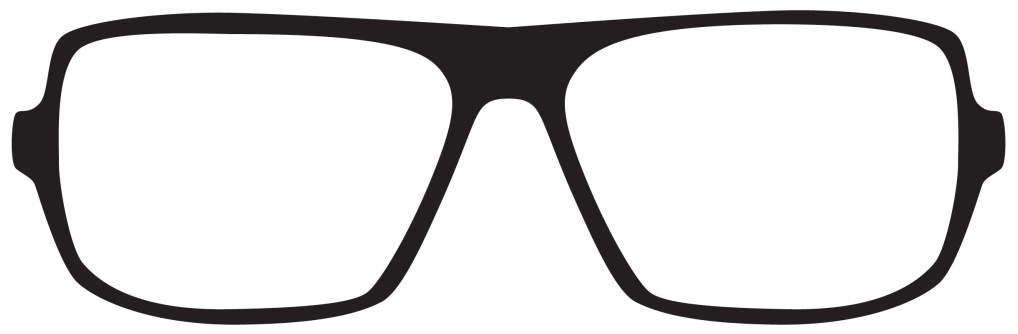




\section{SUMMARY}

Although there is a growing body of research on the role of offence supporting cognitive distortions in child sexual offending, little is known about the origins of these distortions. According to cognitive theory, maladaptive cognitive schemas originating in adverse childhood experiences with caregivers have been hypothesized to underlie these cognitive distortions. This exploratory study investigates Early Maladaptive Schemas (EMS) in child sexual offenders compared to sexual offenders against adults and nonsexual offenders. EMS were measured with the Young Schema Questionnaire, and psychopathy was measured with the Psychopathy Checklist-Revised. Three groups of forensic inpatients - 23 child sexual offenders, 19 sexual offenders against adults and 24 nonsexual violent offenders - were assessed. Multivariate analysis of covariance were used to examine the hypothesized group differences in EMS. Results showed that, after controlling for level of psychopathy, EMS related to Abandonment, Social Isolation, Defectiveness/Shame, Subjugation and Self-Sacrifice were more prevalent in child sexual offenders compared to nonsexual violent offenders. Compared to sexual offenders against adults, child sexual offenders showed a trend to have higher scores on EMS related to Social Isolation. Our findings suggest that early maladaptive schemas may play a role in offending behavior in child sexual offenders and offer the possibility of informing treatment strategies.

\section{INTRODUCTION}

Cognitive distortions concerning child sexual abuse are considered in risk assessment (e.g., Sexual Violence Risk-20; Boer, Hart, Kropp, \& Webster, 1997) and treatment of child sexual offenders (e.g., Mandeville-Norden, Beech, \& Hayes, 2008; Marshall, Marshall, Serran, \& O'Brien, 2011). These distortions denote offence supportive beliefs and attitudes, originating from deep cognitive structures, or schemas (Mann \& Beech, 2003, Ward, 2000; Ward \& Keenan, 1999), and are regarded as contributing to the facilitation and maintenance child sexual offending (Ó Ciardha \& Gannon, 2011). To date, several schemas identified by Ward and Keenan (1999), which they labeled 'implicit theories', are regarded to play a causal role in child sexual offending (Keown, Gannon, \& Ward, 2010). According to Ward and Keenan (1999) implicit theories guide the processing of information and behavior in child sexual offenders. The implicit theories are drawn upon by the child sexual offenders to infer the mental states of the victims, to interpret their behavior, and to make predictions about their future actions and mental states. Examples of offence supportive implicit theories held by child sexual offenders are: children as sexual beings (e.g., 'Children enjoy sex with adults'), entitlement (e.g., 'I am more valuable than others'), dangerous world (e.g., 'Many people are untrustworthy and rejecting'), uncontrollability ('I can't control myself, so I am not responsible'), and nature of harm ('This is really not wrong') (Ward, Gannon, \& Keown, 2006; Ward \& Keenan, 1999; Ward, 2000).

Some studies have provided empirical support for the prevalence of implicit theories in child sexual offenders (e.g., Dawson, Barnes-Holmes, Gresswell, Hart, \& 
Gore, 2009; Marziano, Ward, Beech, \& Pattison, 2006). For example, in a sample of child sexual offenders $(N=22)$, more than $80 \%(n=18)$ had all these cognitive distortions, as assessed by semi-structured interviews (Marziano et al., 2006). However, other studies have produced mixed findings about the prevalence of implicit theories in child sexual offenders (Gannon, 2006; Gannon, Keown, \& Polaschek, 2007; Gannon \& Polaschek, 2005; Gannon, Wright, Beech, \& Williams, 2006). The mixed findings seem to suggest that much remains to be understood about cognitive distortions in child sexual offenders. Findings vary across samples (e.g., intrafamilial vs. extrafamilial offenders), methodologies (e.g., vignettes, questionnaires, and reaction time paradigms) and a gold standard for assessing cognitive distortions in child sexual offenders has not been identified (Keown et al., 2010).

Several authors have argued for a focus on deeper cognitive processes instead of the endproducts of these processes, as most cognitive distortions serve as excuses and post-hoc rationalizations for child sexual offenders (e.g., Ó Ciardha \& Gannon, 2011; Gannon \& Polaschek, 2006; Maruna \& Mann, 2006; Marshall, Marshall, \& Kingston, 2011). Focusing on post-hoc rationalizations, without addressing the underlying cognitive schemas, might not be sufficient in the assessment and treatment of child sexual offenders aimed at decreasing reoffending (Maruna \& Mann, 2006).

Young's framework of early maladaptive schemas (EMS; Young, Klosko, \& Weishaar, 2003) has been offered as a promising approach to understand, assess, and change the deeper lying maladaptive cognitive schemas of sexual offenders (Mann \& Beech, 2003; Marshall, Marshall, Serran, \& O’Brien, 2011; Sigre-Leirós, Carvalho, \& Nobre, 2012). EMS refer to broad, stable and enduring patterns, comprised of memories, emotions, cognitions, and bodily sensations, which are acquired during the course of adverse life experiences from childhood into adulthood, such as rejection, neglect and/or abuse, and loss. EMS guide (emotional) information processing and interpersonal interactions, are triggered by emotional and stressful events, and can result in maladaptive behavior (Young et al., 2003). EMS, in interaction with other factors, such as deviant sexual preferences, may play a role in sexually assaultive behavior (Mann \& Beech, 2003). The framework of EMS expands on the cognitive schema model proposed by Beck, Freeman, and colleagues (1990), by defining specific EMS that lie at the core of personality disturbances (Young et al., 2003). EMS have shown to mediate the association between attachment experiences and psychopathology (Bosmans, Braet, \& van Vlierberghe, 2010; Roelofs, Lee, Ruijten, \& Lobbestael, 2011).

Although recent studies have linked EMS to a variety of psychological disorders and associated maladaptive behaviors including personality disorders (Jovev \& Jackson, 2004), substance abuse (Ball \& Cecero, 2001), psychopathy (Chakhssi, Bernstein, \& de Ruiter, 2012), sexual dysfunction (Quinta Gomes \& Nobre, 2012), and self-reported sexual aggression (Sigre-Leirós et al., 2012), studies on EMS in adult sexual offenders are absent from the literature. However, treatment of EMS has shown favorable results in patients with challenging mental disorders, such as borderline personality disorder and substance abuse (Ball, 2007; Giesen-Bloo et al., 2006; Farrell, Shaw, \& Webber, 2009; Roper, Dickson, Tinwell, Booth, \& McGuire, 2010). Preliminary findings of a randomized clinical trial using Schema 
Focused Therapy (SFT) with personality disordered forensic patients, with violent and sexual offences including child sexual offenders, suggests a reduction in their future violence risk and improves their ability of to be open and vulnerable during therapy sessions (Bernstein et al., 2012).

Prospective studies examining the association between childhood abuse and sexual crimes towards children in adulthood suggest that most victims of sexual abuse do not victimize others in adulthood (Salter et al., 2003). However, abused children subjected to multiple types of early maltreatment, such as sexual abuse, physical abuse, psychological abuse, neglect, and lack of supervision, were significantly more likely to be arrested in adolescence and adulthood for a sexual offence compared to children who had been subjected to sexual abuse only and/or had not been abused as children (Salter et al., 2003; Widom \& Ames, 1994).

Findings from a meta-analysis of 89 (cross-sectional) studies examining risk factors for perpetration of child sexual abuse (Whitaker et al., 2008) also suggest that early adverse experiences are strongly related to child sexual offending. When compared to nonsexual offenders, child sexual offenders were more likely to have a history of physical and sexual abuse (effect size $d=.44$ ). However, when child sexual offenders were compared with sexual offenders against adults, both groups were similar on most developmental risk factors (such as physical and sexual abuse; poor family functioning; controlling and coercive parents; harsh discipline; and parental instability). Although the observed differences between child sexual offenders and sexual offenders against adults were related to psychopathology and generally small in terms of effect sizes (range $d=0.20-0.39$ ), their findings suggest that child sexual offenders were more likely to experience depression, anxiety, and low self-esteem and less likely to display anger/hostility and substance abuse. Furthermore, child sexual offenders, compared to nonsexual offenders, were more likely to experience paranoia/mistrust $(d=.70)$, loneliness $(d=.44)$, difficulty with intimate relationships $(d=.42)$, social skills deficits $(d=.41)$ and sexualized coping $(d=.97)$. Similar findings were reported in two more recent meta-analyses in adult and adolescent sex offenders, where sex offenders were more likely to have a history of abuse than nonsexual offenders (Jespersen, Lalumière, \& Seto, 2009; Seto \& Lalumière, 2010).

Taken together, the findings of these studies support the notion that early maladaptive schemas may play a role in sexually aggressive behaviors against children. Given these earlier findings, one could assume that child sexual offenders, driven by maladaptive schemas related to mistrust/abuse, social incompetence, and insecure attachment, are more likely to isolate themselves from reciprocal adult relationships. This hypothesis is in line with earlier findings that child sexual offenders demonstrated strong feelings of worthlessness and helplessness (Milner \& Webster, 2005), and this is also in line with studies suggesting that negative affect triggered by social isolation and alienation may be associated with deviant fantasies in child sexual offenders (Farmer, Beech, \& Ward, 2011; Looman, 1995; McKibben, Proulx, \& Lusignan, 1994; Proulx, McKibben, \& Lusignan, 1996).

To the best of our knowledge, only one study reported on Young's maladaptive schemas in child sexual offenders (Richardson, 2005). In a sample of sexually abusive adolescents ( $N=54$; mean age $=16$ years), Richardson (2005) found that sexually abusive adolescents against peers were more likely to endorse early 
maladaptive schemas related to entitlement, insufficient self-control, and emotional inhibition compared to sexually abusive adolescents against children.

The main purpose of this exploratory study was to investigate EMS in child sexual offenders compared to sexual offenders against adults and nonsexual offenders. In light of previous findings (Farmer et al., 2011; Looman, 1995; McKibben et al., 1994; Proulx et al., 1996; Ward \& Keenan, 1999; Whitaker et al., 2008), we hypothesized that child sexual offenders are more likely to endorse EMS related to Social Isolation, Emotional Deprivation, and Vulnerability to Harm (Ward and Keenan's Dangerous World schema, 1999) compared to sexual offenders against adults. In addition, following the findings that child sexual offenders were more likely to have a history of neglect, physical and sexual abuse than nonsexual offenders (Jespersen et al., 2009; Salter et al., 2003; Seto \& Lalumière, 2010; Widom \& Ames, 1994), and the differences between child sexual offenders and nonsexual violent offenders in paranoia/mistrust (Whitaker et al., 2008), we hypothesized that child sexual offenders are more likely to endorse EMS related to Abandonment and Mistrust/Abuse compared to nonsexual violent offenders. We used the Psychopathy Checklist-Revised (PCL-R; Hare, 1991) to control for the possible influence of psychopathy because psychopathy is associated with EMS related to mistrust/abuse, inadequate self-control/low frustration tolerance, and autonomy/dominance (Chakhssi et al., 2012). Furthermore, the level of psychopathy varies across different types of sexual offenders and psychopathy may serve as mediator between early adverse experiences and the severity of maladaptive behavior in adulthood, including (sexual) offending (e.g., Porter et al., 2000; Marshall \& Cooke, 1999; Weiler \& Widom, 1996).

\section{METHOD}

\section{Sample}

The sample (all male; $N=66 ; n=23$ child sexual offenders; $n=19$ sex offenders against adults; and $n=24$ nonsexual violent offenders) was recruited from a larger sample of consecutive admissions to a Dutch inpatient forensic psychiatric hospital between 2002 and 2009. All offenders were admitted under the "TerBeschikkingStelling" (TBS)-order. The TBS-order is a judicial measure imposed on offenders who have committed serious offences, carrying a punishment of at least 4 years imprisonment, and who suffer from a mental disorder. The hospital offers a multimodal treatment program to offenders aimed at reducing (the impact of) their mental disorders and the risk of future violence. Patients who met DSM-IV criteria for borderline intellectual functioning (V62.89) or for a disorder belonging to the DSM-IV disorder categories Schizophrenia and Other Psychotic Disorders or Pervasive Developmental Disorders, were excluded from participation in this study. Eligible subjects were classified as child sexual offenders if their index offence and/or prior convictions consisted of at least one sexual contact offence against a victim less than 14 years of age, following the DSM-IV victim age criteria. Among the child sexual offenders (Age: $M=47.0$, standard deviation [SD] =10.1), 22 (95.7\%) had a diagnosis of pedophilia according to the DSM-IV, 10 (43.5\%) had exclusively victimized boys, 5 (21.7\%) had exclusively victimized girls, and $8(34.8 \%)$ had victimized both boys and girls. Nineteen subjects had committed sexual crimes 
against adults but never committed sexual offences against children and were classified as sex offenders against adults (Age: $M=35.8, S D=9.0$ ). The remaining 24 nonsexual violent offenders were extracted from a larger sample of eligible subjects $(n=93)$ using Simple Random Sampling Without Replacement. This strategy ensures that we randomly selected a subset of subjects that has the same probability of being chosen as any other subset in the sample (SPSS Inc., 2004). The subjects had committed a serious violent offence but were never convicted for a sexual offence (Age: $M=38.8, S D=9.6$ ).

\section{TABLE 1}

Mental disorders (DSM-IV)

\begin{tabular}{|c|c|c|c|}
\hline & $\begin{array}{c}\text { CSO } \\
(n=23)\end{array}$ & $\begin{array}{c}\text { SOA } \\
(n=19)\end{array}$ & $\begin{array}{c}\text { NSO } \\
(n=24)\end{array}$ \\
\hline & $\%$ & $\%$ & $\%$ \\
\hline \multicolumn{4}{|l|}{ DSM-IV Axis I Disorder } \\
\hline Substance related disorders* & 30.4 & 78.9 & 75.0 \\
\hline Pedophilia* & 95.7 & 0 & 0 \\
\hline Impulse control disorders & 0 & 0 & 12.5 \\
\hline Mood disorders & 4.3 & 10.5 & 4.2 \\
\hline Anxiety disorders & 8.7 & 10.5 & 8.3 \\
\hline Adjustment disorders & 4.3 & 0 & 0 \\
\hline Attention-deficit disorders* & 0 & 10.5 & 25.0 \\
\hline \multicolumn{4}{|l|}{ DSM-IV Axis II Disorder } \\
\hline Antisocial PD & 17.4 & 47.4 & 33.3 \\
\hline Borderline PD & 8.7 & 26.3 & 16.7 \\
\hline Narcissistic PD & 21.7 & 15.8 & 8.3 \\
\hline Schizotypal PD & 4.3 & 0 & 0 \\
\hline Schizoid PD & 4.3 & 0 & 0 \\
\hline Avoidant PD & 4.3 & 0 & 0 \\
\hline Dependent PD & 0 & 0 & 8.3 \\
\hline PD NOS & 56.5 & 47.4 & 58.3 \\
\hline PD NOS Cluster A traits & 4.3 & 10.5 & 4.2 \\
\hline PD NOS Cluster B traits & 43.5 & 52.6 & 54.2 \\
\hline PD NOS Cluster $C$ traits & 17.4 & 15.8 & 20.8 \\
\hline
\end{tabular}

Note. CSO = Child sexual Offenders; SOA = Sexual Offenders against Adults; NSO = Nonsexual violent Offenders; PD = Personality Disorder; NOS = Not Otherwise Specified. $*$ significant difference between the groups at $p<.05, \chi^{2}$-test (two-tailed). Percentages may add up to more than $100 \%$ because most patients received more than one Axis-I and/or Axis-II diagnosis.

A one-way ANOVA was used to test for age differences among the three groups. Age differed significantly across the three groups $[F(2,63)=8.02, p<$ .001]. Tukey post-hoc comparisons of the three groups indicate that the child 
sexual offenders $(M=47.0,95 \% \mathrm{Cl}[42.7-51.4])$ were significantly older than the sexual offenders against adults $(M=35.8,95 \% \mathrm{Cl}$ [31.47- 40.11]), $p<.001$, and significantly older than the violent, nonsexual offenders $(M=38.8,95 \% \mathrm{Cl}[34.7$, 42.8]), $p=.012$. The age difference between the sexual offenders against adults and the violent, nonsexual offenders was not statistically significant ( $p=.576$ ). Detailed information on DSM-IV Axis I and Axis II diagnoses was extracted from the patients' file charts. DSM-IV diagnoses in the patients' file charts are based on clinical and/or semi-structured interview diagnoses made by psychiatrists and clinical psychologists. The most prevalent DSM-IV Axis I and Axis II diagnoses in the sample and significant differences between the three groups are displayed in Table 1.

\section{Measures}

Young Schema Questionnaire. The Young Schema Questionnaire (YSQ; Young \& Brown, 1994; Dutch version: Sterk \& Rijkeboer, 1997) is a 205-item self-report, Likert type questionnaire designed to assess Early Maladaptive Schemas (EMS), where items can be rated from 1 (completely untrue) to 6 (describes me perfectly). The items are divided among 15 subscales in five schema domains. The first domain is labeled Disconnection/Rejection and consists of the following scales: Emotional Deprivation, Abandonment, Mistrust/Abuse, Social Isolation, and Defectiveness/Shame. The second domain is called Impaired Autonomy/Performance and contains the following scales: Failure to Achieve, Dependence/Incompetence, Vulnerability to Harm, and Enmeshment. The third domain is labeled Impaired Limits and consists of the following scales: Entitlement and Insufficient Self-Control. The fourth domain labeled Other-Directedness and consists of the following scales: Subjugation and Self-Sacrifice. The last domain is labeled Over-vigilance/Inhibition, which consists of the following scales: Emotional Inhibition and Unrelenting Standards. The overall score on each subscale or domain is obtained by calculating the mean of the items in that scale or domain. For all subscales and domains, a higher score reflects a more maladaptive and pervasive core belief. The YSQ has demonstrated favorable reliability and validity in both clinical and non-clinical samples (Lee, Taylor, \& Dunn, 1999; Rijkeboer \& van den Bergh, 2006; Rijkeboer, van den Bergh, \& van den Bout, 2005; Schmidt, Joiner, Young, \& Telch, 1995; Stopa, Thorne, Waters, \& Preston, 2001), and supports the theoretically derived higher-order EMS domains (Schmidt et al., 1995; Waller, Meyer, \& Ohanian, 2001). For the total sample, the mean scores, SDs, and internal consistencies (Cronbach's alpha) for the EMS domains were high and as follows: Disconnection / Rejection $[M=2.15, S D=.80, \alpha=.97]$, Impaired Autonomy / Performance $[M=1.78, S D=.60, \alpha=.91]$, Impaired Limits $[M=2.08, S D=.69$, $\alpha$ $=.90]$, Other-Directedness $[M=2.41, S D=.71, \alpha=.90]$, and Over-vigilance / Inhibition $[M=2.34, S D=.86, \alpha=.91]$.

Psychopathy Checklist-Revised. The Psychopathy Checklist Revised (PCL-R; Hare, 1991; Dutch version: Vertommen, Verheul, de Ruiter, \& Hildebrand, 2002) is a 20-item clinical construct rating scale designed to assess psychopathy in forensic populations. Each item is rated on a scale from 0 to $2(0=$ does not apply, $1=$ applies to some extent, 2 = definitely applies). The PCL-R provides a dimensional total score between 0 and 40 indicating the degree to which the individual matches 
the prototypical psychopath. In the total sample, the mean PCL-R score was 21.83 $(S D=7.02)$, ranging from 8 to 36 . For 56 cases, data on interrater reliability for the PCL-R total score were available. The single measure intraclass correlation coefficient based on absolute agreement for the PCL-R total score was .89. The PCL-R is the most widely used instrument for assessing psychopathy, has been extensively studied and significantly predicts antisocial conduct, including violent and sexual recidivism (Leistico, Salekin, DeCoster, \& Rogers, 2008).

\section{Procedure}

All participants provided written informed consent to participate in the study and approval for the study was obtained from the institutional research review committee. All participants completed the YSQ at admission, as part of the hospital intake procedure. PCL-R assessments were performed during the course of treatment by two independent certified raters.

\section{Statistical analysis}

All analyses were conducted using the Statistical Package for the Social Sciences, version 13 (SPSS Inc., 2005). Normality was tested using the Kolmogorov-Smirnovtest and Levene's test was used for testing homogeneity of variance between groups. Statistical significance for both tests was set at $p<.05$. Assumptions of homogeneity of variance and normality of distribution were not met for the mean scores on the YSQ subscales. Subsequently, the YSQ subscales mean scores were log transformed before analysis. The transformed scores met the assumptions of homogeneity of variances, but six out 15 YSQ subscales did not meet the assumptions of normality. Earlier studies on the assumptions of the parametric analysis of covariance (ANCOVA) have demonstrated that the parametric ANCOVA is robust to violations of either normality or homoscedasticity but not when both assumptions are violated (Olejnik \& Algina, 1984). All subsequent analyses were performed on both the transformed scores and raw YSQ scores. The results were comparable in terms of statistical significance and effect sizes. Hence, the results from the analyses with the raw YSQ scores are reported here. To account for the correlations between the YSQ subscales (Rijkeboer et al., 2005), multivariate analysis of covariance (MANCOVA) was performed to determine which of the three groups differed significantly on the YSQ subscales while controlling for PCL-R scores. When omnibus tests were significant, they were followed by planned comparisons to investigate differences between groups using Sidak correction for multiple comparisons. Between-group effect sizes (Cohen's $d$ ) were calculated using estimated means adjusted for PCL-R scores divided by pooled standard deviations (Cohen, 1988).

\section{RESULTS}

To assess if the differences between the groups in age, and the prevalence of substance use disorders and attention disorders (see Table 1) were related to the YSQ scores, Pearson product-moment correlations were used to measure the association between age and YSQ scores, and point biserial correlations to measure the association between the prevalence of substance use disorders and attention disorders, and YSQ scores. The Other-Directedness schema domain was 
significantly associated with age $(r=.31, p=.01)$, as were the schema subscales Vulnerability to Harm $(r=.40, p<.01)$ and Self-Sacrifice $(r=.32, p<.01)$. There were no significant associations between YSQ scores and substance use disorders and attention disorders in the sample.

The mean scores and standard deviations for the YSQ subscales and higherorder domains for child sexual offenders, sexual offenders against adults, and violent, nonsexual offenders are displayed in Table 2. Overall, child sexual offenders showed the highest mean scores on the YSQ subscales and schema domains, whereas the violent, nonsexual offenders showed the lowest mean scores. The sex offenders against adults displayed the highest PCL-R mean score $(M=24.10, S D=8.15)$, followed by the violent, nonsexual offenders $(M=23.15$, $S D=6.34)$, and the child sexual offenders $(M=18.57, S D=5.62)$. PCL-R total scores differed significantly across the three groups $[F(2,63)=4.29, p=.018]$. Follow-up $t$-tests revealed that child sexual offenders scored significantly lower than sexual offenders against adults $t(40)=-2.60, p=.013$, and that child sexual offenders scored significantly lower than nonsexual violent offenders $t(45)=-2.62$, $p=.012$. There were no significant differences between the mean PCL-R total scores of the sexual offenders against adults and nonsexual violent offenders $t(41)$ $=.67, p=.67$.

After controlling for total PCL-R score and age, the results of the MANCOVA showed that the three groups differed significantly in mean scores on the following schema domains: Disconnection/Rejection $\left[F(2,62)=3.96, p=.024\right.$ (partial $\eta^{2}=$ $.12)]$, Impaired Autonomy/Performance $\left[F(2,62)=3.16, p=.050\left(\eta^{2}=.09\right)\right]$, OtherDirectedness $\left[F(2,62)=7.13, p=.002\left(\eta^{2}=.19\right)\right]$, and Over-Vigilance/Inhibition $\left[F(2,62)=3.45, p=.038\left(\eta^{2}=.10\right)\right]$. There was no significant difference between the offender groups on the Impaired Limits domain $\left[F(2,62)=1.77, p=.180\left(\eta^{2}=\right.\right.$ .07)].

Pairwise comparisons using Sidak correction for multiple comparisons are displayed in Table 2. The child sexual offenders had significantly higher mean scores than the nonsexual violent offenders on four of the five schema domains; effect sizes are large according to Cohen's (1988) criteria: Disconnection/Rejection ( $p=.02$, Cohen's $d=.85)$, Impaired Autonomy/Performance $(p=.047, d=.74)$, Other-Directedness $(p<.01, d=1.05)$, and Over-Vigilance/Inhibition $(p=.03, d=$ .82). Differences in the mean scores of the schema domains between the child sexual offenders and sexual offenders against adults were not significant for any of the domains. Furthermore, the child sexual offenders showed significantly higher mean scores compared to the violent, nonsexual offenders on 5 out of 15 schema subscales, with large to very large effect sizes: Abandonment $(p<.01, d=1.07)$, Social Isolation $(p<.01, d=1.0)$, Defectiveness/Shame $(p=.043, d=.82)$, Subjugation ( $p=.014, d=.94)$, and Self-Sacrifice $(p=.013, d=.89)$. The child sexual offenders did not show significantly higher mean scores compared to the sexual offenders against adults on any schema subscale, although Social Isolation showed a trend towards significance $(p=.066, d=.78)$. In addition, the sexual offenders against adults showed significantly higher mean scores than violent, nonsexual offenders on the schema domain Other-Directedness ( $p=.02, d=.96$ ) and on the schema subscales Subjugation $(p=.017, d=1.08)$, both with large effect sizes. 


\section{TABLE 2}

Unadjusted means (with SDs) on the YSQ of Offenders classified as Child sexual Offenders versus Sexual Offenders against Adults, and Nonsexual violent Offenders

\begin{tabular}{|c|c|c|c|}
\hline \multirow[t]{2}{*}{ Schema domain and subscales } & $\mathrm{CSO}$ & SOA & NSO \\
\hline & $M(S D)$ & $M(S D)$ & $M(S D)$ \\
\hline Disconnection/Rejection & $2.47(.85)^{\mathrm{a}}$ & $2.18(.70)$ & $1.82(.73)$ \\
\hline Emotional Deprivation & $2.64(1.24)$ & $2.55(1.14)$ & $2.28(1.29)$ \\
\hline Abandonment & $2.61(.86)^{b}$ & $2.20(.82)$ & $1.73(.66)$ \\
\hline Mistrust/Abuse & $2.53(.94)$ & $2.48(.73)$ & $2.06(.94)$ \\
\hline Social Isolation & $2.50(1.15)^{b}$ & $1.88(.64)$ & $1.62(.81)$ \\
\hline Defectiveness/Shame & $2.05(.84)^{a}$ & $1.81(.78)$ & $1.42(.67)$ \\
\hline Impaired Autonomy/Performance & $2.00(.60)^{a}$ & $1.78(.45)$ & $1.56(.64)$ \\
\hline Failure to Achieve & $2.13(.81)$ & $2.05(.82)$ & $1.60(.77)$ \\
\hline Dependence/Incompetence & $2.01(.84)$ & $1.82(.46)$ & $1.65(.91)$ \\
\hline Vulnerability to Harm & $2.09(.66)$ & $1.67(.45)$ & $1.60(.60)$ \\
\hline Enmeshment & $1.76(.64)$ & $1.59(.48)$ & $1.38(.48)$ \\
\hline Impaired Limits & $2.28(.64)$ & $2.09(.57)$ & $1.89(.80)$ \\
\hline Entitlement & $2.19(.70)$ & $1.94(.61)$ & $1.77(.82)$ \\
\hline Insufficient Self-Control & $2.36(.65)$ & $2.23(.80)$ & $2.02(.91)$ \\
\hline Other-Directedness & $2.78(.74)^{b}$ & $2.49(.60)^{a}$ & $1.99(.55)$ \\
\hline Subjugation & $2.28(.82)^{a}$ & $2.11(.64)^{a}$ & $1.57(.44)$ \\
\hline Self-sacrifice & $3.29(.86)^{a}$ & $2.88(.65)$ & $2.41(.82)$ \\
\hline Over-Vigilance/Inhibition & $2.69(1.00)^{a}$ & $2.28(.73)$ & $2.05(.71)$ \\
\hline Emotional Inhibition & $2.69(1.19)$ & $2.15(.88)$ & $2.00(.87)$ \\
\hline Unrelenting Standards & $2.70(.96)$ & $2.40(.82)$ & $2.10(.69)$ \\
\hline
\end{tabular}

Note. CSO = Child sexual Offenders; SOA = Sex Offenders against Adults; NSO = Nonsexual violent Offender. ${ }^{a}$ Significant after Sidak correction compared to nonsexual violent offenders at $p<.05 .{ }^{b}$ Significant after Sidak correction compared to nonsexual violent offenders at $p<.01$.

\section{DISCUSSION}

The aim of this study was to contribute to the existing knowledge base on cognitive distortions in child sexual offenders, by using Young's theoretical framework for understanding underlying maladaptive cognitive schemas. First of all, our study revealed a strikingly consistent pattern of early maladaptive schemas among the three offender groups. On four out the five early maladaptive schema domains, child sexual offenders consistently demonstrated the highest scores and the nonsexual violent offenders consistently demonstrated the lowest scores, but the schema domain scores of the child sexual offenders and sexual offenders against adults did not differ significantly from each other. Also, child sexual offenders were significantly more likely to endorse 5 of the 15 early maladaptive schemas 
compared to the nonsexual violent offenders, even after controlling for psychopathy scores. There were no significant differences between the three groups in personality disorder pathology, so this does not account for the overall higher endorsements of early maladaptive schemas in child sexual offenders. These findings suggest that early maladaptive schemas according to Young et al. (2003) are more prevalent in child sexual offenders compared to nonsexual offenders.

Furthermore, in contrast to our first hypothesis, child sexual offenders were not more likely, compared to sexual offenders against adults, to endorse the early maladaptive schemas Social Isolation, Vulnerability to Harm and Emotional Deprivation. Although not significant, child sexual offenders tended to have higher scores on the Social Isolation schema compared to sexual offenders against adults ( $p=.066)$ with a large effect size. This finding, when reached statistical significance, might lend support to the "Dangerous World" cognitive distortion that is highly prevalent in child sexual offenders (Marziano et al., 2006; Ward et al., 2006; Ward \& Keenan, 1999). The perception of being isolated from the world of adults and being different from others may be pertinent to child sexual offenders as it may increase schema-related avoidant strategies, such as seeking distraction or excitement through indulging their sexually deviant fantasies and needs (Looman, 1995; McKibben et al., 1994; Proulx et al., 1996). The lack of further differences between child sexual offenders and sexual offenders against adults in early maladaptive schemas is not surprising, given the earlier findings that child sexual offenders and sexual offenders against adults are similar on most (developmental) risk factors (Jespersen et al., 2009; Whitaker et al., 2008).

The early maladaptive schema Abandonment was found to be more prevalent in child sexual offenders relative to nonsexual violent offenders in this study. People who exhibit the Abandonment schema are triggered by events that they (unconsciously) perceive as similar to adverse experiences of their childhood (Young et al., 2003). As the schema is triggered, they experience strong negative affect (Young, 1994). This finding is in line with earlier findings that adverse early life experiences, such as abuse and neglect, can substantially increase the risk of child sexual offending in adulthood (Salter et al., 2003), and the findings on the association between negative affect and deviant sexual fantasies in child sexual offenders (Looman, 1995; McKibben et al., 1994; Proulx et al., 1996).

Contrary to our expectations, child sexual offenders were not more likely to endorse the Mistrust/Abuse schema compared to nonsexual violent offenders, and compared to sexual offenders against adults. The Mistrust/Abuse schema refers to the expectation of being abused, mistreated, or cheated by others as cognitive sequelae of physical, sexual, and emotional maltreatment during early childhood (Young, 1994). In our sample, all offenders were more likely to endorse the Mistrust/Abuse schema relative to other maladaptive schemas, which suggests they share a common background of early maltreatment experiences.

Feelings of loneliness, lack of intimacy, lack of self-esteem, and problematic interpersonal relationships have been identified as key areas that need attention in the treatment of child sexual offenders (e.g., Hanson, Harris, Scott, \& Helmus, 2007; Hanson \& Morton-Bourgon, 2004; Marshall, Serran, \& Cortoni, 2000; Thornton, Beech, \& Marshall, 2004). The findings of the current study suggest that these areas may be redefined using the schema theory framework. The overall 
schematic structure that emerges suggests that child sexual offenders feel socially isolated, lack a sense of belonging, and are likely to feel lonely as a result. They feel fundamentally inadequate relative to peers, defective, unwanted, and ashamed of themselves. They view the world as a dangerous place where terrible, catastrophic things could happen to them. In relationships, they feel subjugated: they have to be excessively compliant, meet the needs of others, in order to avoid anger, abandonment, or to avoid guilt and/or gain self-esteem. However, we cannot determine whether the early maladaptive schemas endorsed by the child sexual offenders in fact preceded their child sexual offending behavior. The present study is a cross-sectional one, and the endorsement of EMS by child sexual offenders may also be the result of adverse experiences of rejection, stigmatization, and isolation as a consequence of their deviant sexual interests and offending behavior. Notwithstanding this important limitation, the existence of these maladaptive schemas warrants further research into the influence of maladaptive schemas on offending behavior in sexual offenders.

Our preliminary findings also suggest that the early maladaptive schema Subjugation may be relevant for sexual offenders against adults as well. According to Young et al. (2003), Subjugation refers to excessive compliance in relationships at the expense of one's own needs and emotions, in order to gain love and attention. Individuals that are excessively compliant in relationships may eventually manifest outbursts of maladaptive coping responses (e.g., uncontrolled anger, passive aggressive behavior, and substance use) in order to meet their emotional needs, including sexual ones (Young et al., 2003). This is congruent with the findings that sexual offenders against adults are more likely to display anger/hostility and substance use compared to child sexual offenders, and problems in relationships have also been identified as contributing to offending in sexual offenders against adults (e.g., Hanson \& Morton-Bourgon, 2004; Whitaker et al., 2008).

Our findings suggest that early maladaptive schemas can inform the assessment of child sexual offenders. In addition to using a cognitive-behavioral approach that focuses on cognitive distortions and post-hoc rationalizations of (child) sexual offending, Young's schema theory may offer broader understanding of developmental and cognitive processes associated with the underlying schemas (Maruna \& Mann, 2006).

The present study had a number of limitations that need to be considered. First of all, causal inferences cannot be made due to its cross-sectional design. Another limitation was the sole use of an explicit measure of cognitive schemas. Although, the Dutch version of the YSQ is extensively validated (Rijkeboer \& van den Bergh, 2006; Rijkeboer et al., 2005), future studies should add alternatives to selfreport, such as implicit measures to capture early maladaptive schemas (Rijkeboer \& Huntjens, 2007; Leeuwen et al., 2013). Also, we did not use a measure to account for socially desirable responding and/or the tendency to minimize pathology which has been observed previously in (child) sexual offenders (Ward, Hudson, Johnston, \& Marshall, 1997). Another limitation was the small sample size in this study which may have limited the power to detect further differences in YSQ subscales between the offender groups. Given the overall higher mean scores of child sexual offenders, and the magnitude of observed effect sizes, it is possible 
that more differences in YSQ subscales between the offender groups would reach significance in a larger sample. Finally, the current sample was relatively small and consisted mostly of personality disordered forensic inpatients. Thus, the generalizability of our findings remains unclear. However, Dutch forensic hospitals are largely populated by offenders with personality disorders, who are sent to prison in most other Western countries (de Ruiter \& Trestman, 2007). Often a major mental illness is required for admission to a forensic hospital, whereas the latter group makes up only $26 \%$ of the Dutch forensic psychiatric population (van Emmerik, 2001; de Ruiter \& Trestman, 2007). Still, our results suggest that child sexual offenders are more likely to endorse early maladaptive schemas compared with the other offender groups.

In conclusion, our findings suggest that Young's cognitive framework may have added theoretical value in understanding maladaptive schemas that may play a role in (child) sexual offending. Future studies should further clarify the relationship between early maladaptive schemas and offence-related cognitive distortions in sexual offenders. Our findings raise the possibility that Schema Therapy may help some child sexual offenders in dealing with their mistrust of adults, perceptions of neglect, abandonment, and the resulting negative affect, which has often been seen as an important precursor to child sexual offending behavior (e.g., Looman, 1995; McKibben et al., 1994; Proulx et al., 1996; Ward et al., 1997). 
66 | CHAPTER 4 


\section{CHAPTER 5}

Change during Forensic Treatment in Psychopathic versus Nonpsychopathic Offenders

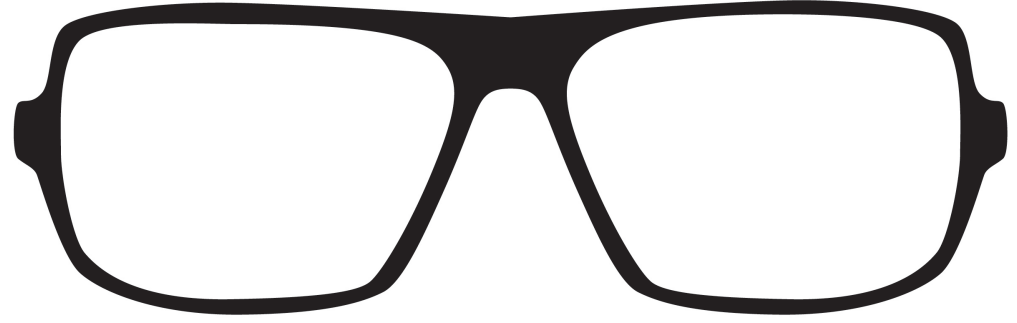




\section{SUMMARY}

Psychopathy in forensic psychiatric patients and other criminal offenders is associated with higher criminal recidivism rates. Moreover, many forensic mental health professionals believe that psychopaths are not amenable to treatment. The present study examines whether patients with psychopathy demonstrate change during forensic psychiatric treatment. Seventy-four personality disordered offenders who had been convicted for serious violence were rated on the PCL-R and assessed repeatedly on risk-related behaviors during 20-months of inpatient forensic treatment. Group- and individual-level analyses showed no significant differences between psychopathic and nonpsychopathic patients on adaptive social behavior, communication skills, insight, attribution of responsibility and self-regulation strategies. However, a subgroup of psychopaths (22\%) deteriorated during treatment with regard to physical aggression, whereas none of the nonpsychopathic patients did. Our findings demonstrate that, contrary to clinical lore, treatment does not make a majority of psychopaths worse, but there are significant differences between psychopaths and nonpsychopaths in treatment responsiveness.

\section{INTRODUCTION}

Psychopathy can be defined as a disorder of personality which includes predatory behavior, emotional detachment, callousness, impulsivity, and persistent antisocial behavior (Hare, 2003; Patrick, 2006). Studies following up on psychopaths after incarceration or hospitalization show that they commit more serious violent crimes, and are more likely to recidivate than nonpsychopaths (Hemphill, Hare, \& Wong, 1998; Hildebrand, Hesper, Spreen, \& Nijman 2005; Leistico, Salekin, DeCoster, \& Rogers, 2008). However, only a few studies have addressed the treatability, aimed at reducing violence risk, of psychopaths.

Approximately $24 \%$ to $35 \%$ of the population in Dutch forensic inpatient settings consists of psychopaths (Hildebrand et al., 2005; Hildebrand \& de Ruiter, 2004 ) and $13 \%$ to $47 \%$ of European and North American forensic psychiatric samples consist of psychopaths (Patrick, 2006). Psychopathic patients pose a tremendous challenge for forensic treatment settings in safeguarding society, especially when forensic mental health professionals are faced with the widely held belief that psychopaths are untreatable (Cleckley, 1988) or that treatment even has adverse effects (Hare, Clark, Grann, \& Thornton, 2000; Rice, Harris, \& Cormier, 1992; Seto \& Barbaree, 1999). This belief has recently been challenged by Salekin (2002) who stated, on the basis of a review of 42 treatment studies, that there is no convincing scientific evidence for the belief that psychopathy is untreatable. More importantly, the review shows that although many mental health professionals and researchers adhere to Hare's definition of psychopathy (1991), only 4 of the 42 studies examined the responsiveness of psychopaths using the current diagnostic standard for assessing psychopathy: the Hare Psychopathy Checklist-Revised (PCLR; Hare, 1991, 2003). Several authors have extensively reviewed PCL-R based research into psychopathy in relation to treatment involvement and found that the few studies addressing this issue provided insufficient evidence to support the view 
that psychopathy is immutable (see Skeem, Monahan \& Mulvey, 2002; Harris \& Rice, 2006) or to support the view that treatment made psychopaths worse (D'Silva, Duggan, \& McCarthy, 2004). On top of that, Seto and Barbaree (1999) who observed that treatment progress in psychopathic sex offenders $(n=224)$ was associated with increases in recidivism, reported in a follow up study using a longer observation period and more recidivism data, that there was no evidence in the follow up data that justified their preliminary conclusion that "treatment causes harm" (Barbaree, 2005).

Results of a recent PCL-R based study, suggest that psychopaths are as likely to benefit from treatment as nonpsychopaths. A prospective study among 381 male offenders mandated to residential drug treatment found that psychopaths who received intensive treatment were over three times less likely to be rearrested at 1 year follow-up than psychopaths who received less intensive treatment (Skeem, 2008). Even though psychopathy was associated with misbehavior and less perceived progress during treatment in this study, the PCL-R scores did not moderate the effect of treatment dose on re-arrest rates. Similar findings were reported in an evaluation of treatment in 871 civil psychiatric patients (Skeem, et al., 2002). In this study, psychopathy did not moderate the effect of treatment involvement and subsequent violence during a post-discharge follow-up of 10 weeks. Moreover, these authors found that civil psychiatric patients with psychopathic traits were three times less likely to be violent when they received an adequate dose of treatment (more than seven sessions during a 10-week period). More recently, similar results have been found in a retrospective study by Olver and Wong (2009). After a 10-year post-treatment follow up, in a sample of 156 PCL-R assessed sex offenders, they found that sex offenders who showed positive therapeutic responses were less likely to recidivate in violent and sexual crimes. Again, this relationship was not moderated by PCL-R scores and/or Sexual Violence Risk scores (Olver \& Wong, 2009).

Taken together, these findings suggest that some psychopaths are amenable to change, but also raise several further questions. First, while psychopaths as a group may show significant reductions in mean level of problems, how many psychopaths show clinically relevant change at an individual level? That is, how many improve, how many stay the same, and how many deteriorate? Second, what dynamic risk factors for violence account for the change in psychopaths? Although, psychopaths share a high likelihood of future criminal behavior and violence, their co-morbid psychopathology is far from homogenous (Brinkley, Newman, Widiger, \& Lynam, 2004; Hildebrand \& de Ruiter, 2004). Hence, the treatment response of psychopaths at a group level may not reflect the development of individual psychopaths during forensic treatment, and more individual focused analyses are necessary to investigate a possible differential treatment within the group of psychopaths. To determine whether individual psychopaths respond to treatment, we use the Reliable Change Index (RCl; Jacobson and Truax, 1991). The RCl was developed to evaluate client change during therapy, and is frequently used in therapy outcome research (Atkins, Bedics, McGlinchey, \& Beauchaine, 2005; McGlinchey, Atkins, \& Jacobson, 2002; Ogles, Lunnen, \& Bonesteel, 2001). The $\mathrm{RCl}$ describes change of individuals during treatment not only in terms of reliable improvement but also in terms of no change and/or reliable deterioration. The $\mathrm{RCI}$ 
measures the reliability of change by taking repeated measurements and measurement unreliability of the instrument used into account. Compared to group level analyses, the $\mathrm{RCl}$ is a more rigorous methodology. To demonstrate reliable change, patients have to change beyond the degree of change that could be explained by measurement unreliability and repeated assessments alone.

In this prospective study, we examined change during long-term inpatient forensic treatment in a sample of male psychopaths and nonpsychopaths classified according to Hare's PCL-R. To obtain a homogeneous sample, only offenders with personality disorders according to DSM-IV-TR (APA, 2000) were included, and patients with major mental disorders were excluded. In light of recent research (Skeem et al., 2002; Skeem, 2008), we hypothesized that psychopaths would show the same treatment responsiveness as nonpsychopaths. We predicted that there would be no group differences in treatment responsiveness, and that on an individual level, similar percentages of psychopaths and nonpsychopaths would show improvement, no improvement, or deterioration according to the $\mathrm{RCl}$. We assessed therapeutic change with the Behavioural Status Index (BEST-Index; Reed, Woods, \& Robinson, 2000), a measure of dynamic, risk-related behaviors. We chose the BEST-Index because it can be administered repeatedly during treatment to assess changes in multiple dimensions of forensic risk, and has shown good reliability and validity in previous studies with forensic patients (Chakhssi, de Ruiter, \& Bernstein, 2010a; Ross et al., 2008; Woods, 2000). Moreover, because the BEST-Index is completed by paraprofessional informants (i.e., psychiatric nurses), it is less susceptible to response bias, such as socially desirable responding, that often affects the responses of forensic patients to self-report questions.

\section{METHOD}

\section{Setting}

This study was conducted at Forensic Psychiatric Center de Rooyse Wissel (dRW), a Dutch maximum security hospital for the treatment of mentally disordered offenders who are hospitalized under the Dutch judicial measure of "TerBeschikkingStelling" (TBS). TBS is a mandatory treatment order imposed on offenders who have committed serious offences, carrying a punishment of at least 4 years imprisonment, and who suffer from a mental disorder according to DSM-IV (Axis I and/or Axis II). The TBS-order is prolonged as long as the court deems the patient a danger to society. The hospital has 229 residential treatment beds for male offenders, divided over three locations.

Treatment objectives and program. The overall treatment objective of $\mathrm{dRW}$ is to reduce future violence risk in mentally disordered offenders by providing inpatient treatment and, in most cases, a stepwise reintegration into society with the aid of correctional- and health service providers. All patients reside in $10-12$ beds high security wards where they are offered a supportive milieu by psychiatric nurses, including motivational interventions (Miller \& Rollnick, 2002), work- and educational programs, individual and/or group psychotherapy by psychologists, psychotherapists, or creative arts therapists (e.g., drama, art, music, or movement therapists), pharmacological interventions by psychiatrists, where indicated, and support in maintaining and building constructive social networks by social workers. 
During this study, the treatment program was tailored to four offender groups: 1) offenders with schizophrenia and other psychotic disorders, 2) personality disordered offenders, 3) sex offenders and 4) intellectually disabled offenders. After an extensive observational and assessment period of three to six months, all personality disordered patients are enrolled in a multimodal treatment program with a cognitive behavioral approach which focuses on relapse prevention (e.g., Laws, Hudson, \& Ward, 2000). During the observation period of the study $(M=20$ months), the psychopathic- as well the nonpsychopathic offenders followed the same treatment protocol. This consisted of group-based weekly sessions of 2 hours led by two cognitive-behavioral therapists. The sessions, which take up to 19 months in length, focus on the patient's criminal behavior, taking responsibility for the committed crime(s), criminogenic needs, and the chain of events that resulted into the offence(s), with a continuing emphasis on the patient's participation to formulate alternative strategies to the behaviors and cognitions which resulted in the offence(s). Given the heterogeneity of problematic behavior within personality disordered offenders, every patient's idiosyncratic criminogenic needs are addressed in successive (and sometimes parallel) therapeutic interventions. For example, group-based Anger Replacement Training (Goldstein, Glick, Reiner, Zimmerman, \& Coultry, 1987) for reactive aggression problems, and/or individual Schema Therapy (Young, 1994) for distorted (antisocial) cognitions, and/or groupbased social skills training (Liberman, DeRisi, \& Mueser, 1989), and/or Creative Arts therapy (see Smeijsters \& Cleven, 2006). During the study period, the average personality disordered patient received 2 hours per week creative arts therapy, 1 hour per week group-based social skills, 1 hour per week individual psychotherapy, 1 hour per week Aggression Replacement Training and 20 hours per week a workand educational program. Also, during the study period psychotropic medications were used in $13.5 \%(n=10)$ of the cases, evenly divided among the psychopaths ( $n$ $=5$ ) and the nonpsychopaths $(n=5)$. Within the psychopathic group: 2 patients used antipsychotics (Pipamperon, 40mg/day; Zuclopentixol, 30mg/day), 1 patient used antidepressants (Trazodon, 100mg/day), 1 patient used anticonsulvia (Depakine $1000 \mathrm{mg} /$ day), and 1 patient used sedatives (Clorazepaatdikalium, $15 \mathrm{mg} /$ day). Within the nonpsychopathic group: 2 patients used antidepressants (Clomipramine, 150mg/day; Paroxetine, 20mg/day), 2 patients used anticonvulsive medication (Depakine $1000 \mathrm{mg} /$ day; Depakine, $1500 \mathrm{mg} /$ day), and 1 patient used psychostimulants (Methylfenidaat, 36mg/day). All the medications were used primarily as an adjunct to the therapeutic interventions and were mostly prescribed to provide symptom relief.

\section{Sample}

The study sample consisted of 74 personality disordered male offenders, admitted to dRW under the TBS-order between March 1, 2000 and May 1, 2007. All patients who stayed at least one year at the hospital were included and were assessed for psychopathy ( $n=160$ ) with the PCL-R (Hare, 1991, 2003; Dutch version: Vertommen, Verheul, de Ruiter, \& Hildebrand, 2002). In order to obtain a more homogeneous sample, patients with borderline intellectual functioning, psychotic-, paraphilic-, and autism spectrum disorders $(n=86)$ were excluded from the study. The resulting sample consisted of 74 personality disordered offenders and 
represented $46 \%$ of the total sample. These patients were divided into two groups, psychopathic offenders and nonpsychopathic offenders, based on the PCL-R cut-off score of greater than or equal to 26 (Hildebrand, de Ruiter, \& de Vogel, 2004; Hildebrand, de Ruiter, \& Nijman, 2004).

Characteristics of the study sample are presented in Table 1. Mean age of the patients was 36.0 years $(S D=8.9)$, and the mean length of stay in the hospital was 3.9 years $(S D=1.9)$. Almost $34(45.9 \%)$ of the patients had committed or attempted homicide, eight (10.9\%) had committed sexual offenses, 26 (35.1\%) had committed violent theft, robbery, or assault, and six $(8.1 \%)$ had committed arson. The classification of personality disorders was conducted on the basis of the raw scores on the Structured Interview for DSM-IV Personality Disorders (SIDP-IV; Pfohl, Blum, \& Zimmerman, 1997; Dutch version: de Jong, Derks, van Oel, \& Rinne, 1996). SIDP-IV scores were unavailable for six patients; three patients refused to be interviewed and three patients had undergone a mental health assessment without a SIDP-IV. For these six patients, we conducted a chart review (including previous psychiatric and psychological reports) using the SIDP-IV criteria for DSM-IV personality disorders.

\section{TABLE 1}

Sample characteristics $(n=74)$

\begin{tabular}{|c|c|c|c|}
\hline & $\begin{array}{l}\text { Sample } \\
(n=74)\end{array}$ & $\begin{array}{c}\mathrm{PCL}-\mathrm{R}<26 \\
(n=47)\end{array}$ & $\begin{array}{c}\text { PCL-R } \geq 26 \\
(n=27)\end{array}$ \\
\hline Age (in years) ${ }^{a}$ & $36.04(8.85)$ & $36.91(8.87)$ & $34.55(8.78)$ \\
\hline Time in treatment (in years) ${ }^{a}$ & $3.86(1.93)$ & $4.19(2.01)$ & $3.30(1.68)$ \\
\hline \multicolumn{4}{|l|}{ Main index offense ${ }^{b}$} \\
\hline Homicide offense (including attempted) & $45.9 \%$ & $46.8 \%$ & $44.4 \%$ \\
\hline Sexual offense & $10.9 \%$ & $12.7 \%$ & $7.4 \%$ \\
\hline Violent theft, robbery or assault & $35.1 \%$ & $29.8 \%$ & $44.4 \%$ \\
\hline Arson & $8.1 \%$ & $10.6 \%$ & $3.7 \%$ \\
\hline \multicolumn{4}{|l|}{ Criminal History } \\
\hline Prior convictions ${ }^{b}$ & $73.0 \%$ & $66.0 \%$ & $85.2 \%$ \\
\hline Age at first conviction ${ }^{a}$ & $20.66(6.17)$ & $21.89(7.00)$ & $18.52(3.58)^{*}$ \\
\hline Prior TBS ${ }^{b}$ & $6.8 \%$ & $0 \%$ & $18.5 \% * *$ \\
\hline \multicolumn{4}{|l|}{ Mental Health History } \\
\hline $\begin{array}{l}\text { Prior contact with mental health } \\
\text { services }^{b}\end{array}$ & $74.3 \%$ & $72.3 \%$ & $77.8 \%$ \\
\hline Age at first mental health contact ${ }^{a}$ & $16.58(9.49)^{c}$ & $17.40(10.03)^{d}$ & $15.27(8.61)^{\mathrm{e}}$ \\
\hline
\end{tabular}

Note. ${ }^{a}$ Oneway ANOVA. ${ }^{b} x^{2}$-test. ${ }^{c} n=57 .{ }^{d} n=35 .{ }^{e} n=22 . *$ significant difference between the groups at $p<.05 . * *$ significant difference between the groups at $p<.01$. 


\section{Materials}

BEST-Index. The BEST-Index (Reed et al., 2000; Dutch version: van Erven, 1999) is a nurse-rated instrument for the assessment of risk-relevant behaviors among forensic psychiatric patients. The instrument contains 70 items divided a priori among three subscales: Risk, Insight, and Communication and social skills. Detailed descriptions of the BEST-Index subscales and item examples are available elsewhere (Chakhssi et al., 2010a; Reed et al., 2000; Ross et al., 2008; Woods, 2000). A previous factor analytic study among 291 Dutch forensic psychiatric patients from dRW revealed an underlying four-factor structure comprising 63 items (Chakhssi et al., 2010a). The four factors of the Dutch version of the BEST-Index were: Social Skills, Insight, Interpersonal Hostility and Physical Violence. The Social Skills factor contains 23 items and is related to social skills and adaptive social behaviors. The Insight factor consists of 21 items measuring the level of insight, into the nature of patient's problems; into antecedent events leading to their current situation; and into attribution of responsibility. The Interpersonal Hostility factor contains 12 items and is related to aggressive and dominant behavior in interpersonal contacts. The fourth factor was labeled Physical Violence, contains 7 items and is related to physically aggressive acts. Each item can be rated on a fivepoint scale ranging from 1 (= worst case) to 5 (= optimal case). Internal consistency of the Dutch version of the BEST-Index and the derived factors was excellent. Cronbach's alpha for the BEST-Index total score was .97, and for the BEST-Index factors internal consistencies were .96 (Social skills factor), .95 (Insight factor), .86 (Interpersonal hostility factor) and .74 (Physical violence factor), respectively. Interrater reliability for the Dutch version was also found satisfactory.

In a sample of 182 raters (psychiatric nurses trained in the BEST-Index), the average measure intraclass correlation coefficient (ICC) for the BEST-Index total score was .84, and for the BEST-Index factors the coefficients were .84 (Social Skills factor), .80 (Insight factor), .82 (Interpersonal Hostility factor) and .81 (Physical Violence factor), respectively. Furthermore, the concurrent and predictive validity of the BEST-Index were supported. Moderate to strong correlations were found between the BEST-Index scales and the Historical Risk Management-20 (HCR20; Webster, Douglas, Eaves, \& Hart, 1997), a widely used and extensively validated instrument for assessing violence risk (for an overview see, Douglas, Guy $\&$ Weir, 2006), as well as between the BEST-Index scores and inpatient violence (Chakhssi et al., 2010a).

PCL-R. The Psychopathy Checklist-Revised (Hare, 1991, 2003; Dutch version: Vertommen et al., 2002) is a clinical construct rating scale designed to assess psychopathy in forensic populations. The PCL-R consists of 20 items, each rated 0 to 2 ( 0 = does not apply, 1 = applies to some extent, 2 = definitely applies). The PCL$R$ yields a dimensional total score between 0 and 40 indicating the degree to which the individual matches the prototypical psychopath. The PCL-R has been extensively studied and significantly predicts general and violent recidivism (Leistico et al., 2008), and it has become the most widely used instrument for assessing psychopathy. In the present study, PCL-R ratings were performed by mental health professionals (i.e., clinical psychologists, psychiatrists and psychotherapists) who all attended certified training in the administration and coding of the PCL-R. 


\section{Procedure}

Approval for the study was obtained from the hospital's executive board and the institutional research review committee.

BEST-Index assessments. Psychiatric nurses were blind to the purpose of the study and to the PCL-R ratings, although they were not blind to descriptive information on psychopathic features used for treatment planning. The psychiatric nurses were all trained in using the BEST-Index and assessed every patient six months after admission, which generally corresponds with the start of the patient's treatment. During their hospital stay, the nurses assessed the patients at six month intervals. Every assessment was performed on their observation of patients' behaviors, and collateral information on relevant behaviors provided in the patient's charts, during the previous six months on the ward. The BEST-Index scoring manual (Woods, 2000; Dutch version: van Erven, 1999) offers detailed rating criteria for every item. Moreover, the scoring manual offers suggestions for acquiring additional information if items are difficult to score (e.g., conducting interviews with patients on relevant topics). Assessments were performed by at least the primary nurse (mentor) on the ward where the patient resided, and one other nurse from the same ward. If the patient was transferred to another ward, then different nurses assessed the patient at the subsequent time points. However, one nurse was always assigned as the primary nurse of the patient. All the patients in the study sample had undergone at least four repeated BEST-Index assessments, which were used for the statistical analyses in the study. The baseline assessment (T1) was performed 4.7 months $(S D=1.2)$ after admission and the mean length of time between the baseline assessment (T1) and the last follow-up assessment used in this study $(\mathrm{T} 4)$ was 1.68 years $(S D=.41)$. The mean scores of the two nurses will be used in this study for the BEST-Index total score (based on 63 items) and factor scores. In total, 206 nurses performed one or more BEST-Index assessments in this study $(M=2.68)$. Interrater reliability for the raters in this study for the different time-points (T1 to T4) was satisfactory. The average measure ICC based on absolute agreement for the BEST-Index total score (based on 63 items) ranged from .76 to $84(M=.82)$. For the BEST-Index factors, the coefficients for the Social Skills factor ranged from .75 to $.83(M=.79)$, the coefficients for the Insight factor ranged from .73 to $.83(M=.80)$, for the Interpersonal Hostility factor they ranged from .80 to $.82(M=.82)$ and for the Physical Violence factor from .63 to $.90(M=$ .81). In the current sample, the reliability coefficients for the BEST-Index total score was .96, and for the BEST-Index factors internal consistencies were .95 (Social Skills factor), .94 (Insight factor), .87 (Interpersonal Hostility factor) and .70 (Physical Violence factor), respectively.

$P C L-R$ assessments. The PCL-R ratings for every patient were carried out in the course of treatment $(M=2.5$ years after admission, $S D=1.88$ ) by two trained and independent raters using file information only. Interrater reliability for the PCL-R total score was satisfactory at .85 (average measure ICC; $n=135$ ). Patient files in the dRW contain criminal records, psychiatric- and psychological reports for the court, violence risk assessments, medical information, treatment progress reports, and reports from psychiatric nurses, social workers, work- and education supervisors. Final PCL-R ratings were based on consensus between the two raters. The PCL-R consensus scores were used in all subsequent data analyses. 
The PCL-R total score is calculated as the sum of all the item scores, when at least 15 of the 20 items are rated. In case of five or less omitted items, the PCL-R total score is adjusted by prorating, according to the procedure stipulated in the PCL-R Manual (Vertommen et al., 2002). Thirty-eight patients had been assessed with the file based procedure as well as with the interview based procedure, and with different raters for the two procedures. The average time between the two procedures was 16.7 months $(S D=9.6)$. The PCL-R mean score for these 38 patients for the file based protocol was $23.97(S D=3.83$, range 15 to 32$)$, and for the interview based protocol $23.94(S D=4.47$, range 13 to 34$)$. A paired sample $t$ test revealed no significant differences between the two procedures for the mean PCL-R total scores $[t(37)=.05, p=.96]$, for the PCL-R Factor 1 scores $[t(37)=.00$, $p=1.0]$ and for the PCL-R Factor 2 scores $[t(37)=.84, p=.41]$. The average measure ICC between the scores derived from the file based- and interview based procedure was found to be .73 for the PCL-R total score, .68 for the PCL-R Factor 1 scores and .73 for the PCL-R factor 2 scores. Although, file based procedures may not capture the complete PCL-R Affective and Interpersonal features, these preliminary findings suggest that the differences between either procedures most likely are small in our sample.

\section{Statistical analysis}

Differences in demographic and clinical variables between the psychopathic and nonpsychopathic offenders were tested using Analysis of Variances (ANOVA) and contingency tables with the Pearson Chi-Square test or Fisher exact tests, when appropriate. A repeated measures ANOVA was used to examine the changes on the BEST-Index total and subscale scores between T1 and T4. Group (psychopathic versus nonpsychopathic offenders) was used as between-subjects factor and the repeated assessments on the BEST-Index (T1, T2, T3, T4) as within-subjects factor.

Individual Change. To examine individual change, we used the $\mathrm{RCl}$ developed by Jacobson and Truax (1991). As stated earlier, the RCl addresses whether patients' change during treatment exceeds the change that would be expected based on measurement error alone. The $\mathrm{RCl}$ controls for the degree of difference in scores from pre- to post-test as a result of measurement unreliability. The $\mathrm{RCl}$ is defined as (Jacobson \& Truax, 1991; p. 14):

$$
\frac{X_{2}-X_{1}}{\sqrt{2(S E)^{2}}}
$$

where $X_{1}$ is a subject's pretest score, $X_{2}$ is a subject's posttest score and SE is the standard error of measurement. SE is calculated by multiplying the standard deviation of the pretreatment group with the square root of 1 minus the reliability of the assessment measure. The denominator, also described as $\mathbf{S}_{\text {diff, reflects "the }}$ amount of difference which one could expect between two scores, obtained on the same test by the same individual, as function of measurement error alone" (Christensen \& Mendoza, 1986; p. 307). Under the assumption of normality, 68\% of change attributable to measurement error will fall within the range of -1 and $1 S_{\text {diff }}$ , and $95 \%$ of change attributable to measurement error will fall within the range of 
-1.96 and $1.96 \mathrm{~S}_{\text {diff }}$. RCl's larger than 1.96 would be unlikely to occur $(p<.05)$ without actual change. In this study, patients with a negative reliable change $(\mathrm{RCl}<$ -1.96) will be labeled as reliably deteriorated, patients within the band of no reliable change $(-1.96 \leq \mathrm{RCl} \leq 1.96)$ will be labeled as uncertain change, and patients with a positive reliable change $(\mathrm{RCl}>1.96)$ will be labeled as reliably improved. All analyses are performed using the Statistical Package for the Social Sciences, version 13 (SPSS Inc., 2005).

\section{RESULTS}

In this sample, the most prevalent personality disorder (PD) for both the nonpsychopathic and the psychopathic group was Antisocial PD $(44.7 \%$ versus 81.5\%; see Table 2).

\section{TABLE 2}

DSM-IV Classifications

\begin{tabular}{|c|c|c|c|c|}
\hline & $\begin{array}{l}\text { PCL-R }<26 \\
(n=47)\end{array}$ & & $\begin{array}{l}\text { PCL-R } \\
(n=27\end{array}$ & \\
\hline & $n$ & $\%$ & $n$ & $\%$ \\
\hline \multicolumn{5}{|l|}{ DSM-IV Axis I Disorder } \\
\hline Substance related disorders & 37 & 78.7 & 25 & 92.6 \\
\hline Attention deficit and disruptive behavior disorders & 10 & 21.3 & 7 & 25.9 \\
\hline Impulse control disorders & 7 & 14.9 & 2 & 7.4 \\
\hline Mood disorders & 5 & 10.6 & 0 & 0 \\
\hline Anxiety disorders & 4 & 8.5 & 1 & 3.7 \\
\hline Learning disorders & 0 & 0 & 1 & 3.7 \\
\hline Any Axis I disorder & 40 & 85.1 & 26 & 96.3 \\
\hline Any Axis I disorder without Substance abuse & 22 & 46.8 & 10 & 37.0 \\
\hline \multicolumn{5}{|l|}{ DSM-IV Axis II Disorder } \\
\hline Antisocial PD & 21 & 44.7 & 22 & $81.5^{* *}$ \\
\hline Borderline PD & 6 & 12.8 & 7 & 25.9 \\
\hline Narcissistic PD & 0 & 0 & 7 & $25.9 * *$ \\
\hline Paranoid PD & 4 & 8.5 & 2 & 7.4 \\
\hline Schizoid PD & 4 & 8.5 & 2 & 7.4 \\
\hline Obsessive Compulsive PD & 2 & 4.3 & 1 & 3.7 \\
\hline Avoidant PD & 2 & 4.3 & 0 & 0 \\
\hline Dependent PD & 1 & 2.1 & 0 & 0 \\
\hline Any PD & 23 & 48.9 & 24 & 88.9 \\
\hline
\end{tabular}

Note. PD = Personality Disorder. ** Significant difference between the groups at $p<.01, x^{2}$-test (twotailed). 
Group differences on DSM-IV Axis I and Axis II diagnosis were found in the prevalence of Antisocial personality disorder $\left[\mathrm{x}^{2}(d f=1, N=74)=9.54 ; p=.003\right]$ and Narcissistic personality disorder $\left[\left(x^{2}(d f=1, N=74)=13.46 ; p<.001\right]\right.$. Tests of between group differences on demographic and judicial variables shows that the psychopathic group was significantly younger at the time of their first conviction $[F(1,73)=5.44, p=.022]$ and they had a significantly higher number of prior TBSorders $\left[\mathrm{x}^{2}(d f=1, N=74)=9.35 ; p=.005\right]$.

The mean PCL-R score was $23.10(S D=6.40)$. For the PCL-R facets as defined by Hare $(2003)$, the mean score for the Interpersonal facet was $4.0(S D=2.24)$, $5.73(S D=1.82)$ for the Affective facet, $5.65(S D=2.15)$ for the Impulsive Lifestyle facet, and $5.85(S D=2.70)$ for the Antisocial facet (see Table 3).

\section{TABLE 3}

PCL-R mean and facet scores for the nonpsychopathic and psychopathic patients

\begin{tabular}{lcc}
\hline Scale & $\begin{array}{c}\text { PCL-R }<26 \\
(\mathrm{n}=47)\end{array}$ & $\begin{array}{c}\text { PCL-R } \geq 26 \\
(\mathrm{n}=27)\end{array}$ \\
\cline { 2 - 3 } & Mean (SD) & Mean (SD) \\
\hline PCL-R Total & $19.35(4.32)$ & $29.61(3.51)$ \\
Facet 1: Interpersonal & $3.34(2.14)$ & $5.15(1.97)$ \\
Facet 2: Affective & $5.19(1.78)$ & $6.67(1.52)$ \\
Facet 3: Impulsive Lifestyle & $4.83(1.86)$ & $7.07(1.88)$ \\
Facet 4: Antisocial & $4.74(2.48)$ & $7.78(1.89)$ \\
\hline
\end{tabular}

Note. All differences between psychopaths and nonpsychopaths in PCL-R mean scores are significant at $p<.001$ (two-tailed, $t$ test).

The mean scores on the BEST-Index total were $234.74(S D=32.50)$. The mean scores for the BEST-Index factors at T1 were 91.26 (SD = 14.56) for the Social Skills factor, $62.69(S D=14.89)$ for the Insight factor, $47.48(S D=7.48)$ for the Interpersonal Hostility factor, and $33.30(S D=2.70)$ for the Physical Violence factor (see Table 4).

Change during treatment at the group level. The results of the repeated measures ANOVA are presented in Table 4. The means and standard deviations at the four time points are presented for the psychopaths (PCL-R $\geq 26$ ) and nonpsychopaths (PCL-R < 26) on the BEST-Index factors and total score.

On the BEST-Index total score, there was no difference between the scores of psychopaths and nonpsychopaths (no main effect for Group: $F(1,72)=.19, p=$ .668). The BEST-Index total score improved during treatment for both the psychopaths and nonpsychopaths (main effect for Time: $F(3,216)=5.54, p<$ .001). The interaction between Group and Time did not reach statistical significance, $F(3,216)=1.82, p=0.144$. For the BEST-Index Social Skills factor, the sphericity assumption was not met and the degrees of freedom were corrected using the Huynh-Feldt correction $(\varepsilon=.93)$. The main effect of Time for the Social 
Skills factor was statistically significant, $F(2.80,201.45)=4.13, p=.009$ (with Huynh-Feldt correction).

\section{TABLE 4}

Mean scores and standard deviations on the different time points for the psychopathic and nonpsychopathic patients

\begin{tabular}{llllll}
\hline Scales & PCL-R & Mean T1 (SD) & Mean T2 (SD) & Mean T3 (SD) & Mean T4 (SD) \\
& score & & & & \\
\hline BEST Total & $<26$ & $232.77(34.52)$ & $245.54(34.09)$ & $243.92(32.11)$ & $256.12(33.53)$ \\
& $\geq 26$ & $238.17(28.95)$ & $238.02(26.26)$ & $245.28(34.16)$ & $245.82(37.55)$ \\
Social skills & $<26$ & $89.60(15.97)$ & $94.76(13.27)$ & $94.19(13.00)$ & $97.84(14.67)$ \\
& $\geq 26$ & $94.17(11.40)$ & $93.76(9.83)$ & $96.76(11.53)$ & $96.98(13.20)$ \\
Insight & $<26$ & $61.53(15.51)$ & $69.97(15.50)$ & $68.30(15.42)$ & $74.12(16.29)$ \\
& $\geq 26$ & $64.70(13.80)$ & $66.48(12.46)$ & $71.46(16.90)$ & $70.81(17.13)$ \\
Interpersonal & $<26$ & $48.26(7.15)$ & $47.80(7.44)$ & $48.01(6.92)$ & $50.30(5.70)$ \\
Hostility & $\geq 26$ & $46.11(7.96)$ & $45.39(7.14)$ & $45.37(8.16)$ & $46.46(8.15)$ \\
Physical & $<26$ & $33.37(2.56)$ & $33.02(2.87)$ & $33.43(2.73)$ & $33.86(1.86)$ \\
Violence & $\geq 26$ & $33.18(2.97)$ & $32.39(3.01)$ & $31.69(4.11)$ & $31.56(3.60)$ \\
\hline
\end{tabular}

The main effect of Group was not significant, $F(1,72)=.27, p=.606$, and the Group $x$ Time interaction for the Social Skills factor was also not significant, $F$ $(2.80,201.45)=1.43, p=.238$ (with Huynh-Feldt correction). For the BEST-Index Insight factor, the main effect of Time was significant, $F(3,216)=9.43, p<.001$. Again, the main effect of Group was not significant, $F(1,72)=.00, p=.970$, and the Group $x$ Time interaction for the Insight factor was also not significant, $F$ (3, 216) $=2.18, p=.096$.

For the BEST-Index Interpersonal Hostility factor, the psychopaths scored lower than the nonpsychopaths (main effect of Group: $F(1,72)=4.30, p=.042$ ), indicating that psychopaths showed more interpersonally hostile behavior. Follow-up $t$ tests revealed that psychopaths scored lower than nonpsychopaths at Time $4, t$ $(40.84)=2.161, p=.037$. There were no differences between the mean scores of psychopaths and nonpsychopaths at the other time points [Time $1, t(72)=1.197$, $p=.235$; Time 2, $t(72)=1.360, p=.178$; Time 3, $t(72)=1.479, p=.144]$. There was no main effect of Time $[F(3,216)=1.59, p=.193]$, and no significant Group $x$ Time interaction $[F(3,216)=.33, p=.806]$ for the Interpersonal Hostility factor. For the Physical Violence factor, the main effect of Group was significant, $F(1,72)=4.78, p=.032$. Follow-up $t$ tests revealed that psychopaths scored lower than nonpsychopaths at Time $4, t(34.11)=3.104, p=.004$, meaning that the psychopaths showed more physical violence at Time 4. And again, there were no differences at the other time points [Time $1, t(72)=.286, p=.776$; Time $2, t(72)$ $=.895, p=.374$; Time 3, $t(39.43)=1.96, p=.057]$. The main effect of Time was not significant for the Physical Violence factor, $F(3,216)=1.69, p=.170$, but the 
Time $x$ Group interaction was significant, $F(3,216)=3.96, p=.009$. Post hoc analyses using repeated measures analysis of variance with polynomial contrasts for Group $x$ Time suggest that this interaction is due to a significant linear trend $(F(1,72)=9.546, p=.003)$, indicating that the change slope over the four time points for the two groups are in opposite direction of each other. There was no main effect of Time and no significant simple effects, for the Physical violence scores, but an inspection of the means shows a small improvement in Physical violence scores for the nonpsychopaths, as well as a slight deterioration in these scores for the psychopaths.

\section{TABLE 5}

Reliable Change for the BEST total and subscales from T1 to T4 $(n=74)$

\begin{tabular}{|c|c|c|c|c|c|c|}
\hline \multirow[t]{2}{*}{ Scales } & \multicolumn{2}{|c|}{ Reliable deterioration } & \multicolumn{2}{|c|}{ Uncertain change } & \multicolumn{2}{|c|}{ Reliable improvement } \\
\hline & $n$ & $\%$ & $n$ & $\%$ & $n$ & $\%$ \\
\hline \multicolumn{7}{|l|}{ BEST Total } \\
\hline $\mathrm{PCL}<26$ & 3 & 6.4 & 16 & 34.0 & 28 & 59.6 \\
\hline$P C L \geq 26$ & 2 & 7.4 & 15 & 55.6 & 10 & 37.0 \\
\hline \multicolumn{7}{|l|}{ Social Skills } \\
\hline $\mathrm{PCL}<26$ & 3 & 6.4 & 24 & 51.1 & 20 & 42.6 \\
\hline $\mathrm{PCL} \geq 26$ & 3 & 11.1 & 15 & 55.6 & 9 & 33.3 \\
\hline \multicolumn{7}{|l|}{ Insight } \\
\hline $\mathrm{PCL}<26$ & 4 & 8.5 & 14 & 29.8 & 29 & 61.7 \\
\hline$P C L \geq 26$ & 2 & 7.4 & 15 & 55.6 & 10 & 37.0 \\
\hline \multicolumn{7}{|c|}{ Interpersonal hostility } \\
\hline $\mathrm{PCL}<26$ & 3 & 6.4 & 36 & 76.6 & 8 & 17.0 \\
\hline$P C L \geq 26$ & 3 & 11.1 & 18 & 66.7 & 6 & 22.2 \\
\hline \multicolumn{7}{|c|}{ Physical violence } \\
\hline $\mathrm{PCL}<26$ & 0 & 0 & 43 & 91.5 & 4 & 8.5 \\
\hline $\mathrm{PCL} \geq 26$ & $6^{* *}$ & 22.2 & 19 & 70.4 & 2 & 7.4 \\
\hline
\end{tabular}

Note. $* * p<.01$ (Fisher exact tests, two-tailed).

Change during treatment at the individual level. Table 5 presents the results of individual level change using the $\mathrm{RCl}$, including the number and percentage of psychopaths and nonpsychopaths that reliably deteriorated, showed uncertain change or reliably improved. Differences in the observed and expected distribution of reliable change in psychopaths and nonpsychopaths were tested using the Fisher exact tests for association in the $3 \times 2$ contingency table. The Fisher exact tests were not statistically significant for the BEST-Index total score $(p=.166)$, for the Social Skills factor $(p=.623)$, for the Insight factor $(p=.114)$, and for the Interpersonal Hostility factor $(p=.683)$. The Fisher exact tests was statistically 
significant for the Physical Violence factor $(p=.003)$. A post-hoc Fisher exact tests in the $2 \times 2$ contingency table (deterioration versus uncertain change/improvement) revealed that a greater proportion of psychopaths reliably deteriorated on the Physical Violence factor during treatment $(p=.002)$.

No association was found between psychopathy and reliable improvement in the post-hoc Fisher exact tests (deterioration / uncertain change versus improvement), $p>$.999. Post hoc analyses were performed to assess whether the difference between the psychopaths who reliably improved and the psychopaths who showed no reliable change and/or deteriorated could be explained by different scores on the four PCL-R facets. No significant differences within the psychopathic group were found on the PCL-R facet mean scores (deterioration / uncertain change versus improvement). Also, no significant differences within the psychopathic were found on the sample characteristics (see Table 1) when we compared the psychopathic patients who improved versus psychopathic patients who showed no change or showed deterioration during treatment on the BEST-Index total and factors.

\section{DISCUSSION}

In the present study, we examined treatment responsiveness in a sample of psychopathic and nonpsychopathic offenders in an inpatient forensic psychiatric hospital. We hypothesized that there would be no group differences in treatment responsiveness, and that psychopaths and nonpsychopaths would show similar percentages of reliable individual change. To the best of our knowledge, no earlier studies have examined individual change in forensic patients, where the level of psychopathy was assessed with Hare's PCL-R.

Does treatment makes psychopath worse? In contrast to clinical lore, treatment in this study did not make all or a majority of psychopaths worse. In terms of individual change, psychopaths and nonpsychopaths showed overall the same pattern of reliable deterioration on the BEST total score. Acts of physical violence were an exception, however, nearly a quarter (22\%) of psychopaths showed reliable deterioration, whereas nonpsychopaths showed no reliable deterioration in physical violence during treatment. This finding is in line with previous studies demonstrating that psychopaths are more likely to show inpatient aggression during hospitalization (e.g., Hildebrand et al., 2004) and that psychopaths are more likely to show negative behaviors during treatment than nonpsychopaths (Hobson, Shine, \& Roberts, 2000). Hobson and colleagues examined 104 inmates with the PCL-R and a negative behavior checklist during 6 months in a prison-based therapeutic community. They found that psychopathy was significantly associated with poor adjustment to the therapeutic community as demonstrated by disruptive behaviors during therapy sessions, on the ward, and during other activities (e.g., "manipulates others"; "tells lies"; "inflated sense of self-importance"). On the basis of their findings, the authors suggest that the problematic behaviors of psychopaths could be explained by a lower level of treatment involvement due to the interpersonal and affective features of psychopathy. Similar to these findings, Morrissey, Mooney, Hogue, Lindsay, and Taylor (2007) found that psychopathy, and especially the Interpersonal and Affective facets, predicted inpatient movements from medium to 
high hospital security conditions in a sample of intellectual disabled offenders $(N=$ 73). In our sample - which was rather small to conduct post hoc analyses - we did not find differences on the four facet scores between the psychopaths who deteriorated and those who improved. Given the findings of brain imaging studies in psychopaths (Raine, Lencz, Bihrle, LaCasse, \& Colletti, 2000), an additional explanation could be that these - deteriorating - psychopaths may have poorer functioning in brain regions (e.g., prefrontal cortex) involved in regulating and controlling aggressive behavior. Overall, there were significant differences on physical violence (in group and individual level analyses), and on interpersonal hostility (in group level analyses only).

Do psychopaths improve with treatment? First, we found no differences between psychopaths and nonpsychopaths on the BEST-Index scales at baseline assessment. Whilst differences between psychopathic and nonpsychopathic patients on the baseline measurement BEST-Index scales are to be expected, few studies have addressed pre-treatment differences between PCL-R psychopaths and nonpsychopaths. We could retrieve only one PCL-R based study in a comparable forensic psychiatric sample (Hildebrand \& de Ruiter, 2012) addressing pretreatment differences between psychopaths $(n=27)$ and nonpsychopaths $(n=70)$ on several indirect and self-report measures of dynamic risk, including insight and aggression. Hildebrand and de Ruiter found no pre-treatment differences between psychopathic and nonpsychopathic patients on measures of negative attitudes, egocentrism, impulsivity, and lack of insight. They did, however, find pre-treatment differences between psychopaths and nonpsychopaths on 2 out of 6 indicators of anger/hostility. Second, in line with previous studies our results suggest that some psychopaths do improve with treatment (Olver \& Wong, 2009; Skeem et al., 2002; Skeem, 2008). The group and individual level analyses suggest that both psychopaths and nonpsychopaths are responsive to inpatient forensic treatment, demonstrated by significant changes on the BEST-Index total score, and on the BEST-Index factors measuring Social Skills and Insight. Psychopaths, as well as nonpsychopaths, seem to change on BEST-Index scales that measure adaptive social behavior, communication skills, level of insight and attribution of responsibility. More specifically, approximately one third of the psychopaths showed reliable improvement on the BEST-Index total score, and the BEST-Index factors measuring Social skills and Insight. Again, these findings contradict the widely held belief that psychopaths cannot change (Cleckley, 1988). On the other hand, there is some indication that the nonpsychopathic patients improved somewhat more than the psychopathic patients. Overall, both the group and individual level analyses showed few significant differences between psychopaths and nonpsychopaths in terms of change.

Overall, our findings confirm our hypothesis that within the group of psychopaths treatment responsiveness varies and underlines our notion that individual variability within psychopaths is masked in group level analyses. Consistent with some previous research (Olver \& Wong, 2009; Skeem et al., 2002; Skeem, 2008) our findings demonstrate that treatment does not make all or most psychopaths worse, but also that there are differences between psychopaths and nonpsychopaths in terms of change during treatment. 
Limitations of the study. The findings of the current study should be considered with several limitations in mind. The generalizability of the findings is limited to inpatient male forensic psychiatric patients with personality disorders. The study should be replicated in larger and different samples (e.g., forensic psychiatric patients with psychotic disorders) to assess the strength of our findings. In addition, the observation period in this study ( $M=1.67$ years) is roughly one third of the regular treatment duration under the Dutch TBS order ( $M=5.9$ years; Wartna, el Harbachi, \& Essers, 2006). Research with longer observation periods should be performed to determine if psychopathic patients remain responsive to forensic psychiatric treatment over time. One limitation of the current study is the lack of a no-treatment control group, inherent to forensic clinical outcome studies where withholding treatment is seen as unwarranted and/or unethical. This methodological limitation indicates that any observed differences, or in this study the lack of differences, in change between the psychopathic and nonpsychopathic offenders could be due to natural changes in the subjects over time rather than to effects of treatment. However, giving the documented behaviors in psychopaths of poor adjustment to therapeutic communities (e.g., Hobson et al., 2000; Ogloff, Wong, \& Greenwood, 1990), increased aggressive behaviors during treatment (e.g., Hildebrand et al., 2004) and higher attrition rates (e.g, Ogloff et al., 1990; Olver \& Wong, 2009), we believe that improvements shown by psychopathic patients in our study are unlikely to be due to solely to the passage of time. However, firm conclusions on the observed change in psychopathic and nonpsychopathic offenders can only be drawn in relation to a no-treatment control group. Another limitation was that we could not compare the psychopathic and nonpsychopathic patients on actual therapy involvement and intensity of treatment. No reliable data on treatment intensity and involvement were available for the observation period. However, the treatment programs for personality disordered offenders in the dRW consist generally of the same treatment modalities (see Method section) and there is no separate treatment policy for psychopathic patients. Future research should take specific treatment objectives and strategies into account to examine the differences in responsiveness between psychopathic and nonpsychopathic patients. Future research should also aim at examining how treatment responsiveness of psychopathic patients is related to change in future violence risk. At this time, we cannot say what bearing these findings have on future offending behavior.

Furthermore, we used a behaviorally-based measure of forensic risk, the BEST index. Although, we had a large sample of raters $(n=206)$ in this study with satisfactory interrater reliability, we cannot be certain if and how habituation of the staff to patients' behavior effected the observed change. However, given the large sample of raters and different raters over time who rated each patient, we believe that possible habituation effects are minimal. Also, we do not know to what extent inferences based on observable behaviors correspond to changes in underlying personality constructs such as lack of empathy, lack of remorse, or impulsivity (Blair, 2003; Cooke \& Michie, 2001). There is considerable evidence suggesting that callous-unemotional traits form the temperamental core of psychopathy, that these traits are under strong genetic control, and that they are rooted in neurobiological deficits in limbic (e.g., amygdala) and other brain regions (Blair, 2003; Raine et al., 2000). We do not know whether behavioral improvements 
exhibited by some psychopaths during treatment are associated with corresponding changes in cognitive, affective, or neurobiological mechanisms that are thought to underlie psychopathy. Future studies should attempt to measure changes in these domains during forensic treatment, using experimental paradigms such as tests of implicit cognition (Gray, McCulloch, Smith, Morris, \& Snowden, 2003), psychophysiological responding, and brain imaging procedures.

We also cannot say with certainty that some of the improvement seen in psychopathic patients was not "faked". Psychopaths are known to be extremely adept at impression management. For this reason, we avoided using self-report measures of treatment responsiveness that are known to be susceptible to this kind of manipulation. However, it is still conceivable that some of the apparent improvements in areas such as insight and social skills were based partly or entirely on the pseudo-adaptation of psychopaths who had learned to produce socially desirable behaviors to meet the expectations of their treatment providers. On the other hand, the fact that the vast majority of psychopathic patients (> $77.8 \%$ ) in the study showed little or no physical aggression or interpersonal hostility during the nearly two-year course of treatment suggests that many of these patients are able to exert genuine self-control over their behavior, at least within a structured forensic psychiatric setting. Nevertheless, we will only be able to make more definitive statements about the genuineness of these changes when we examine the long-term outcomes of these patients with respect to recidivism.

Clinical implications Our findings, as well as those of some previous studies (e.g, Barbaree, 2005; D'Silva et al., 2004; Olver \& Wong, 2009; Salekin, 2002; Skeem et al., 2002; Skeem, 2008), suggest that treatments should not be routinely withheld from forensic patients based on their high PCL-R scores. Although psychopaths do show higher rates of recidivism than other forensic patients, this does not imply that they are untreatable. Indeed, a growing body of research suggests that psychological services, such as psychotherapy and addiction treatment programs, can be beneficial for many forensic patients, including psychopathic ones. In the future, it will be important to predict which psychopathic patients are able to benefit from forensic treatments, versus those who deteriorate or remain unchanged, so that treatment decisions can be made on an empirically supported and cost-effective basis. However, at this time, the blanket decision to withhold treatment from psychopathic patients in general does not seem to be supported by research findings. 
$84 \mid$ CHAPTER 5 


\section{CHAPTER 6}

Treating the Untreatable: A Single Case Study of a Psychopathic Patient Treated with Schema Therapy

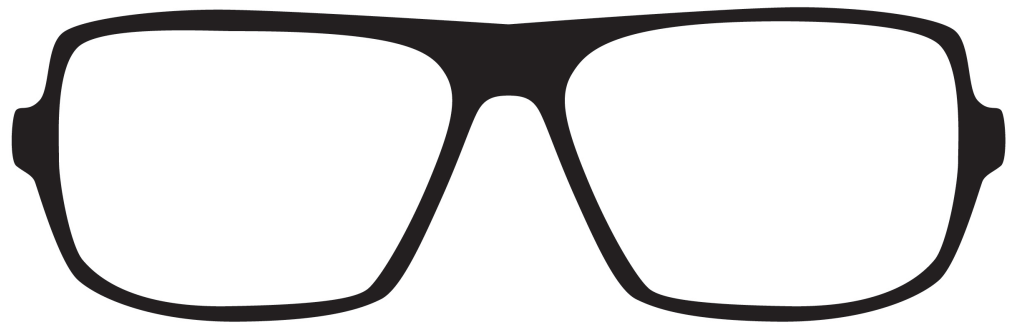




\section{SUMMARY}

From its first conceptualization in modern psychiatry, psychopathy has been considered difficult if not impossible to treat. Schema Therapy (ST) is a psychotherapeutic approach that has shown efficacy in patients with borderline personality disorder. ST has recently been adapted for personality disordered forensic patients, including patients with high levels of psychopathy. The present case study examines the process of individual ST, combined with movement therapy and milieu therapy by the nursing staff, in a forensic patient with psychopathic features (Psychopathy Checklist-Revised total score $=28.4$ ). The patient had been sentenced to a mandatory treatment order in relation to a violent sexual assault. We assessed change using independent assessments of psychopathic traits, cognitive schemas, and risk-related behaviors over the 4-year treatment period and a 3-year follow-up. We also assessed the quality of the working alliance. Reliable change analyses showed significant improvements in psychopathic traits, cognitive schemas, and risk-related outcomes. At 3 years posttreatment, the patient was living independently outside of the forensic institution without judicial supervision and he had not reoffended. While many questions remain about the effectiveness of treatment for psychopathic patients, our study challenges the view that they are untreatable.

\section{INTRODUCTION}

Psychopathy is viewed as a severe form of antisocial personality disorder (ASPD) with greater risk of violence than ASPD (e.g., Coid \& Ullrich, 2010), characterized by a lack of empathy and remorse, self-aggrandizement, superficial charm and poor behavioral controls. The Psychopathy Checklist-Revised (PCL-R; Hare, 1991, 2003) is a reliable and valid assessment tool for measuring psychopathy (Neumann, Hare, \& Newman, 2007), and provides a description of psychopathy which involves two factors: Factor 1 comprises the interpersonal and affective traits of emotional detachment and a manipulative interpersonal style, and Factor 2 comprises the behaviors of an impulsive and antisocial lifestyle (Hare, 2003). The emotional detachment in psychopathy is thought to be related to innate neurobiological deficits in emotion processing (i.e., callous-unemotional traits; Blair, 2003; Viding, Blair, Moffitt, \& Plomin, 2005), whereas impulsivity, hostility, and antisocial behavior may be best understood as an emotional adaptation to adverse experiences in childhood (e.g., Caspi et al., 2002; Huizinga et al., 2006; Weiler \& Widom, 1996).

In forensic settings, the prevalence of psychopathy varies from $13 \%$ to $47 \%$ (Patrick, 2006) and the disorder has been surrounded by therapeutic pessimism. Many experts believe that psychopathic characteristics are difficult, if not impossible, to ameliorate (Harris \& Rice, 2006), and the findings of some studies suggest that treatment makes psychopaths recidivate more (Hare, Clark, Grann, \& Thornton, 2000; Rice, Harris, \& Cormier, 1992; Seto \& Barbaree, 1999). However, more recent studies have supported the idea that some psychopathic patients may benefit from (inpatient) cognitive behavioral treatment programs (Chakhssi, de 
Ruiter, \& Bernstein, 2010b; Hildebrand \& de Ruiter, 2012; Olver \& Wong, 2009; Skeem, Monahan, \& Mulvey, 2002). However, the effectiveness of these approaches is difficult to evaluate due to the limitations of this research, such as a lack of randomized clinical trials (see Salekin, Worley, \& Grimes, 2010).

Schema therapy (ST; Rafaeli, Bernstein, \& Young, 2011; Young, Klosko, \& Weishaar, 2003) is an integrative therapy for personality disorders that combines elements of cognitive, behavioral, psychodynamic, and humanistic/experiential forms of therapy. ST targets chronic emotional and cognitive maladaptive patterns, called "Early Maladaptive Schemas" (EMS), which originate in adverse childhood experiences and early temperament. EMS are repeating themes about oneself and one's relationships that affect emotional processing, influence interpersonal interactions, and guide behavior. The primary objective of ST is ameliorating EMS, replacing maladaptive coping responses with adaptive ones, and the modification of transient, state-related manifestations of EMS, termed "schema modes" (Young et al., 2003). The latter are activated when specific EMS are triggered by specific situations, leading to overwhelming emotions and maladaptive coping responses that account for rapid changes in mood and behavior often observed in personality disordered patients (e.g., Kellogg \& Young, 2006; Lobbestael \& Arntz, 2010). ST has shown effectiveness in three clinical trials of non-forensic outpatients with borderline personality disorder (BPD), including two randomized clinical trials (Farrell, Shaw, \& Webber, 2009; Giesen-Bloo et al., 2006) and one open trial (Nadort et al., 2009). A substantial proportion of the patients in these studies were judged to be in remission from their BPD or to have made clinically significant improvement.

Bernstein and colleagues (Bernstein, Arntz, \& de Vos, 2007) adapted ST for forensic patients. They hypothesized that antisocial and psychopathic patients make prominent use of five schema modes that involve overcompensatory coping styles: attempts to con and manipulate ("conning and manipulative mode"), selfaggrandizement and devaluation of others ("self-aggrandizer mode"), attempts to bully and intimidate ("bully and attack mode"), focusing of attention to detect a hidden threat or enemy ("paranoid over-controller mode"), and cold, calculated aggression aimed at eliminating a threat or rival ("predator mode"). Furthermore, schema modes were conceptualized as the psychological risk factors for patients' offending behavior (Bernstein et al., 2007). Recent research supports the schema mode model in patients with antisocial personality disorder and psychopathy (Chakhssi, Bernstein, \& de Ruiter, 2012; Lobbestael, Arntz, Cima, \& Chakhssi, 2009), including the hypothesized link between schema modes and offending (Keulen-de Vos et al., 2012).

Given its goal of forming a genuine emotional connection with the patient, and altering the patient's core personality traits, ST represents a departure from other cognitive-behavioral treatments for psychopathy (e.g., Wong \& Hare, 2005) that assume that changing psychopathic personality features is impossible due to these patients' serious emotional deficits (Blair \& Mitchell, 2009; Yang \& Raine, 2009). In contrast, ST views psychopathic patients on a continuum in their capacity for emotional relatedness. This notion is consistent with findings from recent studies showing considerable heterogeneity within psychopathic populations (e.g., Brinkley, Newman, Widiger, \& Lynam, 2004; Hildebrand \& de Ruiter, 2004), and suggesting 
that some psychopathic features may be linked to insecure attachment styles (e.g., Frodi, Dernevik, Sepa, Philipson, \& Bragesjø, 2001; van IJzendoorn et al., 1997) and early trauma (e.g., Farrington, 2006; Lang, af Klinteberg, \& Alm, 2002; Marshall \& Cooke, 1999; Poythress, Skeem, \& Lilienfeld, 2006; Weiler \& Widom, 1996), and not solely to innate neurobiological factors.

Empirical studies that have actually examined psychopaths' treatment responsiveness are scarce, and to our knowledge, studies using a psychotherapeutic approach to further our understanding of psychopathy are absent from the literature. The aim of this study is to contribute to the current literature by exploring the use of ST, more specifically Bernstein and colleagues' (2007) forensic adaptation of ST, in the understanding and treatment of a patient with psychopathic traits. We describe the 4-year ST treatment and 3-year follow-up of a 25-year old patient with psychopathic features (pre-treatment PCL-R total score $=28.4$ ) who was admitted to a Dutch forensic psychiatric hospital. First, we will present the ST treatment process. Second, we present scores of the working alliance and the clinical progress of the patient as repeatedly measured during treatment by the PCL$\mathrm{R}$, a self-report measure of EMS, and measures of risk-related behaviors. Finally, we discuss implications for the treatment of individuals with psychopathy.

\section{METHOD}

\section{Setting}

The case study took place at forensic psychiatric hospital "de Rooyse Wissel" (dRW) in The Netherlands. dRW is a maximum security hospital for the treatment of mentally disordered offenders sentenced to involuntary treatment under the Dutch TBS-order. The TBS-order is a mandatory treatment order imposed on offenders who suffer from a mental disorder or a developmental disorder, and who have committed a serious offense, carrying a sentence of at least four years imprisonment (de Ruiter \& Hildebrand, 2003).

\section{Patient}

Andy (not his real name) is a white Dutch man who was 25 years old when he was admitted to dRW. He was sentenced to 3 years imprisonment, followed by mandated treatment under the TBS-order (de Ruiter \& Hildebrand, 2003), after committing a sexually violent offense.

Andy grew up as the only child from a marriage of young parents. Andy recalled being beaten by his authoritarian father on a daily basis, usually for some misbehavior. His mother, on the other hand, was a quiet and compliant woman who tried to protect him from the abusiveness of his father. Andy and his mother were both regularly physically abused. By the age of 8 , Andy's behavior became problematic. He was caught regularly committing thefts (e.g., shoplifting, taking money from his parents and/or family). At a later age, he got involved with antisocial peers resulting in criminal behaviors such as vandalism, thefts (e.g., car radio) and committing assaults (e.g., towards other youth). At the age of 11, juvenile court placed Andy in a correctional care center for youth because of problematic behavior at home and at school. At the age of 14, Andy returned to his 
family but quickly resumed his defiant and oppositional behavior. The frequent physical assaults by his father returned. By the age of 16, Andy reported increasing difficulty controlling his aggressive impulses. At this time, he started to experiment with soft- and hard drugs. He did not complete secondary education. He took several unskilled jobs, but had difficulty maintaining them. He reported that he often did not show up for work or came to work intoxicated. His first conviction occurred at age 17 for aggravated assault. His criminal behavior continued with convictions for vandalism, theft, drug possession and aggravated assault. At the age of 18, his parents divorced. Andy lived alternately with his father and his mother, but he did not get along with either of them. Andy stayed with friends until he managed to acquire his own apartment.

At the age of 19, Andy committed a sexual offense. Together with a fellow perpetrator, he kidnapped a female stranger and raped her. After his arrest, he did not admit committing the sexual offence and tried to lay the blame on the victim ("it was consensual sex", "she was a prostitute") and on the fellow perpetrator ("being forced by my friend to rape the girl").

DSM-IV-TR classification. Andy was assessed with the Structured Interview for DSM-IV Personality Disorders (SIDP-IV; Pfohl, Blum, \& Zimmerman, 1997). Classification for Axis-I disorders was performed by a hospital psychiatrist. Andy's full scale IQ on the Wechsler Adult Intelligence Scale (WAIS-III; Wechsler, 2001) was 85 (verbal $I Q=83$, performance $I Q=91$ ). Andy fulfilled the DSM-IV-TR criteria (American Psychiatric Association, 2000) for alcohol abuse, cocaine abuse, amphetamine abuse (all in remission), and for antisocial personality disorder with borderline and narcissistic traits.

\section{Psychotherapist}

Dr. TK, a certified cognitive-behavioral and ST therapist with extensive forensic psychotherapy experience, conducted the therapy. Although ST is usually given twice per week for patients with severe personality disorders (Young et al., 2003), it was given once a week because the patient was chosen as a "training case" to give the therapist the opportunity to learn to practice ST. Some therapy sessions were videotaped, and viewed in supervision, giving the therapist feedback from a ST supervisor (Dr. DPB) and peer-therapists. Two of the therapist's videotapes were randomly selected and rated for therapist competency using the Schema Therapy Rating Scale (STRS; Young \& Fosse, 2005). The STRS was rated by an independent rater, a certified ST therapist, who had no prior knowledge about the therapist, the patient or the study. The therapist scored at least 4 on a 1 to 6 scale on all areas of the STRS, thus achieving the international standard for competency in the practice of ST as established by the International Society for Schema Therapy.

\section{Measures}

The Psychopathy Checklist-Revised (PCL-R; Hare, 1991) is a reliable and valid assessment tool of the clinical construct of psychopathy (Neumann, Hare, \& Newman, 2007). The PCL-R consists of 20 items, divided over four facets: Interpersonal, Affective, Impulsive Lifestyle, and Antisocial. Each item is rated on a scale from 0 to $2(0$ = does not apply, 1 = applies to some extent, 2 = definitely applies). Previous research has shown that the PCL-R can be rated reliably in 
samples consisting of Dutch personality disordered offenders (Chakhssi et al., 2010b).

The Young Schema Questionnaire (YSQ; Young \& Brown, 1994) is a self-report instrument designed to assess early maladaptive schemas. It consists of 205 items, each rated from 1 (completely untrue) to 6 (describes me perfectly). The items are divided among 15 subscales in five higher-order domains. The first domain is labeled Disconnection/Rejection, the second domain is called Impaired Autonomy/Performance, the third domain is labeled Impaired Limits, the fourth domain is labeled Other-Directedness and the fifth domain is labeled Overvigilance/Inhibition. Previous research has shown that the YSQ has adequate reliability and validity in both clinical and non-clinical samples (Lee, Taylor, \& Dunn, 1999; Rijkeboer, van den Bergh, \& van den Bout, 2005; Schmidt, Joiner, Young, \& Telch, 1995; Stopa, Thorne, Waters, \& Preston, 2001).

The Behavioural Status Index (BEST-Index; Reed et al., 2000) is a structured observational measure that contains 63 items, divided over four scales: Insight, Social Skills, Interpersonal Hostility and Physical Violence. Each item can be rated on a five-point scale ranging from 1 (= worst case) to 5 (= optimal case). Previous research has shown that the BEST-Index can be used reliably and is significantly associated with institutional aggression and risk of future violence (Chakhssi, de Ruiter, \& Bernstein, 2010a).

The Historical Clinical Risk-management-20 (HCR-20; Webster, Douglas, Eaves \& Hart, 1997) is specifically designed for assessing the risk of future violence among persons with mental disorders. It consists of 20 items, each rated from 0 to 2 ( 0 = does not apply, 1 = applies somewhat, 2 = definitely applies). The HCR-20 is intended to structure professional judgment about violence risk and raters are asked to make a final risk judgment of low, moderate or high. Studies across diverse samples have shown that the HCR-20 is strongly related to (violent) recidivism, even when controlling for PCL-R scores (Guy, Douglas, \& Hendry, 2010). Previous research has shown that the HCR-20 can be rated reliably in samples consisting of Dutch personality disordered offenders (Chakhssi et al., 2010a).

The Working Alliance Inventory - Observer, Short version (WAI-O-S; Tracey \& Kokotovic, 1989; Tichenor \& Hill, 1989) is a 12-item observer-rated measure, ranging from 1 (never) to 7 (always), consisting of three subscales: Bond (presence of a therapeutic bond), Tasks (agreement on therapeutic strategies and techniques), and Goals (agreement on the goals of therapy). Previous studies showed that the WAI-O-S can be rated reliably (Myers \& Hayes, 2006), and that the observer-rated alliance was a stronger predictor of therapy outcome than the therapist-rated alliance (Horvath \& Symonds, 1991).

Patient's pre-treatment scores on the measures. Andy obtained a pretreatment PCL-R total score of 28.4. Andy received scores of 4 out of 8 on the Interpersonal facet, 7 out of 8 on the Affective facet, 6 out of 10 on the Impulsive Lifestyle facet, and 8 out of 10 on the Antisocial facet of the PCL-R. According to these scores, Andy showed a high level of a manipulative and arrogant interpersonal style and had a history of frequent and diverse antisocial behaviors. Also, he showed some features of deficient affective experience, such as lack of remorse and failure to accept responsibility for his actions and he had also shown impulsive and irresponsible behavior. Table 1 provides the pre-treatment scores for 
the YSQ, the BEST-Index total and scale scores, and the PCL-R total and facet scores.

\section{Procedure}

Informed consent. Andy participated in the case study after giving written informed consent. He understood, and agreed that we could examine his hospital files, including his criminal records, and make use of the notes from the therapist, therapy process, PCL-R, YSQ, HCR-20 and BEST-Index for the purpose of a single case study. Additional informed consent was obtained for re-administrating the PCL$\mathrm{R}$ and for scoring the videotaped therapy sessions.

Therapy process. The ST treatment process was documented on the basis of the therapist's case notes, as well as extensive discussions with her. Several drafts of the manuscript were sent to the therapist for review. She had many comments and suggestions that helped us to describe the therapy process in detail.

Outcome assessment. The YSQ is a self-report instrument, the HCR-20 is completed by trained psychologists and the BEST-Index is completed by nurses, resulting in independent assessments pre-, mid- and post ST treatment. The PCL-R was coded using an interview (videotaped by one rater and viewed by another) and a review of the patient's file. For the purpose of the case study, we treated the initial PCL-R assessment as a pre-treatment score and re-administered the PCL-R again six months after the end of ST treatment. The second PCL-R was scored by Dr. CdR, a certified PCL-R trainer. Dr. CdR and Andy did not know each other prior to this assessment, and Dr. CdR had no prior knowledge of findings from the earlier PCL-R assessment, BEST-Index ratings, YSQ scores, HCR-20 scores or ST progress. Again, for the purpose of the case study, and to be stringent, after the scoring of the second PCL-R, Dr. CdR did a consensus scoring with the two original raters of the first PCL-R score. The WAI-O-S was coded by three Masters-level students who watched the beginning, the middle and the end of four randomly selected (out of 10 available) videotaped sessions. The rated videotaped sessions corresponded with the beginning (one session, first year), middle (two sessions, second and third years) and end of the therapy (one session, fourth year). The coders had no knowledge about the study, the therapist or the patient. The segments (i.e., beginning, middle, end) were randomly presented to the three coders. Coders based their ratings on viewing each segment. Interrater agreement of the coders

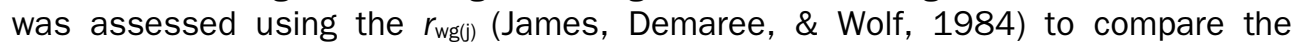
observed variance in multiple raters' rating of a single subject. The interrater agreement for the WAI-O-S total score was high at .98.

\section{RESULTS}

\section{Therapy Process}

Andy began ST 6 months after admission, continued therapy for 4 years, and was discharged from the hospital by court on conditional release approximately one year after completing ST. His mandated treatment order was terminated unconditionally after court review about three years after completing ST. 
Initial phase of therapy (first year approximately).

Initial impressions, aggression and impulse control training.

Dr. TK's initial contact with Andy was for aggression management treatment. Andy suffered from stress and frustration on the ward, which resulted in frequent outbursts of verbal aggression. In the first psychotherapy sessions, Andy presented himself as "macho": a tough, charming, and dominant young man. He was not emotionally detached but rather angry and over-controlling. If not interrupted, he could speak nonstop throughout entire sessions, without room for the psychotherapist. Getting him to stop and experience his emotions was extremely difficult. He was reluctant to discuss his abuse history and refused to talk about the details of his offense. He was often angry about events that had happened on the ward, or between him and his treatment coordinator (i.e., the head of the treatment unit where he resided). After Dr. TK felt that she had gained some trust, she used empathic confrontation and limit setting when the patient's anger or aggression threatened to escalate. An example of how the psychotherapist used limit setting during these first sessions (i.e., 10th session) is illustrated by the following:

Andy: I can't trust anyone on my ward, patients nor nurses. They don't keep their agreements, they are not straight with me, they lie to me about a lot of things, I can't rely on them anymore. Therefore I have to be in charge, take control of everything because I won't let them abuse me... [his demeanor felt threatening and intimidating]

Dr. TK: Andy, please stop! (makes a "stop" sign with her hand) I now see a side of you that takes over control, not only on the ward, but also in this therapy session. I have seen this side in previous sessions also and I know it has an important survival and protective function for you, but it's so dominating now that it obstructs the therapy. This side makes it impossible for me to have a conversation with you. So please listen to me for a moment.

Andy: Why do I have to stop talking? I'm frustrated about this! I am...

Dr. TK: Stop, Andy, listen to me! I know you're frustrated and I really understand that and want to hear more about your frustrations. But first, I want to stop that side of you that's controlling this therapy session... I can't make a genuine connection with you and with your feelings, your vulnerable side, that lies behind this controlling protector side .... Can you recognize this controlling side?

Andy (irritated and embarrassed): Well, this is not a side, it's normal that I take control when other people lie to me!

Dr. TK: I can understand that you take control if you can't trust others but I think this is a way of coping you learned when you were very young, because you never could trust an adult.

Dr. TK had to interrupt Andy several times before he stopped. She did not find it easy to use limit setting because she felt that the patient could become aggressive if he experienced the limit setting as an attack. Andy slowly began to recognize his controlling, intimidating side. After Dr. TK used limit setting, there were glimpses of a more vulnerable, anxious side, which seemed easily threatened. Andy described a violent childhood and he appeared to have strong needs for fairness, autonomy, consistency, and predictability. Andy stated that he was startled when the therapist interrupted him firmly but later during the same session, he could acknowledge his controlling side and its protective function for him.

After the use of limit setting in these first sessions, which led to further insights into the origins of Andy's strong need for control, combined with behavioral 
interventions aimed at aggression regulation, Andy gained greater control over his anger and impulsivity. For example, Dr. TK taught Andy to recognize triggers (mostly perceived authoritarian behavior) and how to cope with these triggers by using stopsigns, avoiding or escaping from triggering situations. Now that he attained his immediate goals, he saw no reason to continue treatment. Notable in Andy's case and with psychopathic patients in general, is that clear treatment goals, measurable in behavioral terms, have to be formulated. Andy's treatment coordinator therefore suggested that the next goal should be to develop a schema focused case conceptualization and offense scenario analysis: thus, a description of the factors involved in his index offense (i.e., the sexual offense that led to the TBS-order) using schemas and schema modes as the conceptual framework.

In the Netherlands, an offense scenario analysis, based on Laws' relapse prevention model for sexual offenders (1999) is a core component of forensic psychiatric treatment, to help offenders recognize risk factors for their offenses and take responsibility for them (Buschman \& Van Beek, 2003). In offense scenario analysis, the patient and psychotherapist systematically examine the events leading up to and culminating in the patient's offense, using a cognitive-behavioral framework (i.e., antecedents, cognitions, emotions, behaviors, and consequences) to understand them (Buschman \& Van Beek, 2003; Dowden, Antonowicz, \& Andrews, 2003; Laws, 1999). For Andy, it was decided to incorporate the offense scenario analysis into the initial phase of ST, to create an integrated approach. Also, the treatment of substance use was integrated into the ST by using schema mode concepts.

Schema Therapy assessment and case conceptualization, including offense scenario analysis.

The initial phase of ST involves uncovering the patient's EMS, maladaptive coping responses, and schema modes, their childhood origins, and their relationship to the patient's problem behaviors. This process ends with a case conceptualization, which the psychotherapist shares with the patient. In ST, the assessment process is collaborative, where the psychotherapist teaches the patient the "language" of EMS and modes, and they work together to develop a shared understanding of the patient's problems using these concepts. Because in Andy's case, the offense scenario analysis was incorporated into ST, and there were many sessions where the focus was on the therapeutic alliance and working with the patient's schema modes (see below), the assessment and case conceptualization phase took much longer than is normally the case, occupying the first year.

The assessment began by exploring Andy's childhood experiences, first examining the origins of his EMS, and subsequently, of his modes. Dr. TK used the concepts of EMS and modes to explain his problems, such as drug abuse, relationship conflicts, and criminal and violent behavior. It is best to first approach the patient's childhood history, and only later his offenses. Once the patient comes to understand the effects of his childhood experiences, it is easier for him to understand his offenses. An example of how the EMS and modes are explored is illustrated by the following:

Andy: My father was Hannibal Lector, a real psychopath. When I came home, one minute late, he would hit me and lock me up in my room for the rest of the day. 
So when I grew up, I decided to take the upper hand, I became a member of a street gang and I learned to attack before anyone could attack me. On the street I felt safer than at home

Dr. TK: So your father hit and abused you a lot, you have already given me other examples. You developed schema's and modes as a consequence. You learned at a young age that you couldn't trust your father and that your mother couldn't stand up for you, so you felt very unsafe. This is the schema mistrust/abuse: you learned to mistrust people first-off. And later on you tried to cope with your mistrust by taking the upper hand and become an aggressive chap. You developed what we call the "bully and attack mode" to survive.

During this initial phase the psychotherapist is highly structured, making systematic inquiries about his childhood temperament, experiences with parents, and other aspects of the childhood environment. Dr. TK consistently but gently pushed him to examine his emotions. When she did so, there was more vulnerability evident in his voice and his appearance changed.

At first, Andy was barely able to talk about his index offense; he could not say the word "rape" out loud. He admitted he had tried to lay blame on his accomplice. Now, he admitted to being the main perpetrator. He appeared to feel shame, confusion, and regret about having raped a woman and having used force and intimidation. With tears in his eyes, he asked, "How can I have done this to a woman?" He had experienced a complete loss of control during his offense, and felt guilty that he had not stopped himself when at a certain moment he had realized that what he was doing was wrong. He said he deserved to pay the rest of his life for what he had done to his victim.

After nearly a year, the case conceptualization was complete. The psychotherapist shared a simplified version of the case conceptualization (see Figure 1) with the patient, using only schema modes. The left of Figure 1 represents overcompensatory modes present in Andy's case; the rectangle shape represent "protector" modes, protecting the child modes behind a defensive wall.

Figure 1. Schema mode model used in Andy's therapy.

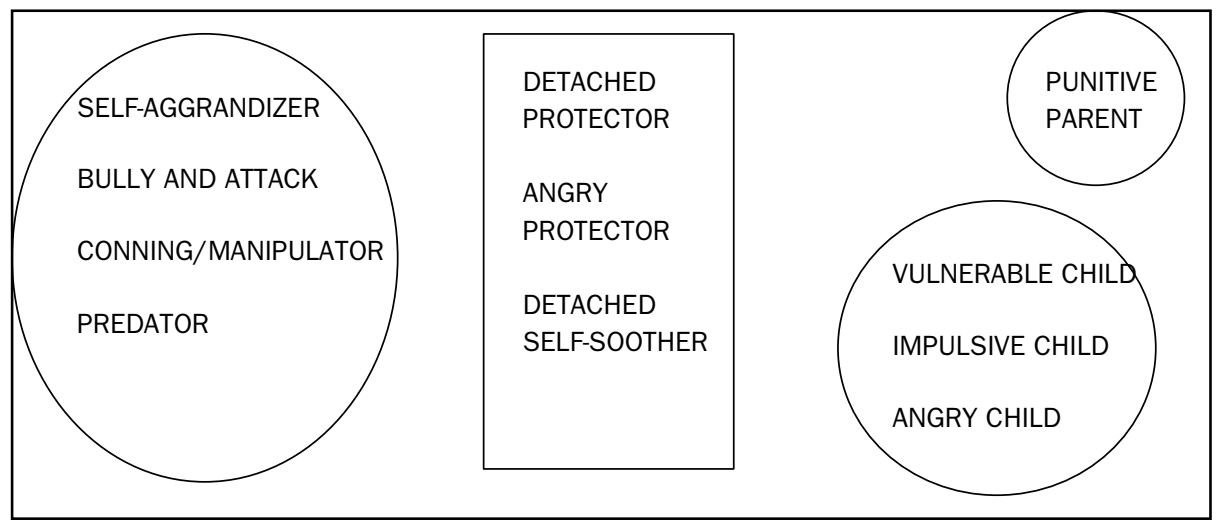


Most forensic patients find this concept easier to understand than EMS. As a result, we focus on schema modes, much more than EMS, in working with forensic patients (Bernstein et al., 2007).

Having finished the offense scenario analysis and case conceptualization, Andy was able to make a formal presentation about his offense scenario, with Dr. TK's support, at a meeting with his treatment team and significant family members. This is a common procedure in Dutch forensic hospitals, an important step towards taking responsibility for the offense. In his own words, Andy described the schema modes that played a central role in his offenses, including his aggressive behavior towards girlfriends, and the "random violence" he had displayed as part of a gang. He provided a detailed analysis of his index offense in terms of an unfolding sequence of schema modes, each mode described in terms of associated feelings, thoughts, behaviors, intentions, and consequences (Figure 2). For example, after his girlfriend left, Andy's feelings of abandonment were triggered (abandonment schema). He felt anxious and powerless (vulnerable child mode). In order to cope with these feelings, Andy got high on drugs (detached self-soother mode). However, as a consequence of the drug use his anger towards his girlfriend became uncontrollable (angry child mode) and he set out to seek revenge (predator mode).

Figure 2. Andy's crime scenario analysis in terms of a sequence of schema modes.

\section{Events leading up to the crime \\ Event Girlfriend breaks up the relationship and has left me \\ (abandonment schema)}

\section{Self-Aggrandizer}

Feelings

Thoughts

Behavior

Intention

Consequences

\section{Self-Soother}

Feelings

Thoughts

Behavior

Intention

Consequences
Glad, powerful (Entitlement schema)

Well, if it is a sure thing that you are going to leave me, I am going to have a nice evening, I am going to use again (XTC and coke), I am going to party.

1. Substance abuse

2. Call a friend to go out

Feeling more powerful/better, freeing myself from my girlfriend (who keeps telling me what to do), decide for myself what I want to do.

In a daze, intoxicated from substances. I revert back to feelings of powerlessness.

Powerless, afraid to be alone (Abandonment schema) I cannot stand to be alone at home, I have nobody left, usually I swap my former girlfriend for a new one and now I don't have a new one, I want to hide my powerlessness.

1. More substance abuse

2. Going out and party

Forget about the abandonment, not feeling anxious / powerless.

More intoxicated, sweating, tingling.

\section{Angry child/ Predator}

Feelings Angry, furious (Insufficient Self-control schema)

Thoughts You cannot leave me, I am going to get you back or I will kill you.

Behavior Demand friend to drive me to my girlfriend's place.

Intention Take revenge, get power over my girlfriend back. 
Consequences Stress: the closer I get to her place, the more tense I get because she gives as good as she gets, I am figuring out scenario's how I can regain control over the situation.

\section{Bully and Attack / Predator}

Event

Feelings

Thoughts

Behaviour

Intention

Consequences

Event

Feelings

Thoughts

Behaviour

Intention

Consequences
Friend says that we are lost and I do not believe him.

Angry, powerful (Entitlement schema).

You obstruct my plans and you are lying.

Threaten, intimidate, I said: I will kill you.

Take my anger out on my friend.

Feeling frustrated, because my friend does not know the directions and it does not help if I threaten, intimidate him.

A girl is walking down the street, I ask her for directions and she tells me that she does not know.

Disappointed.

This sucks. She did not know the directions either. She probably doesn't live in the neighbourhood.

I thanked her for her effort in helping me to find the directions. Find the directions to my girlfriend's house.

Continuing to threaten my friend, getting angrier.

\section{Predator / Self-Aggrandizer}

Event

Feelings

Thoughts

Behavior

Intention

Consequences

Feelings
Thoughts
Behaviour
Intention
Consequences

Event

Feelings

Thoughts

Behaviour

Intention

Consequences

Event

Feelings

Thoughts

Behaviour

Intention

Consequences
Doubts about the intentions of the girl (she is still walking around, so she must know her way around in this neighbourhood), feelings of being fooled (Mistrust/Abuse schema)

Angry

You fooled me, although I asked it nicely

Threatening by saying: You do know the neighbourhood, you live here! She replies that she does not know the directions. I want to know the directions from her Frustrated, became really angry, I wanted to make it very clear to her: do not try to fool me

Very angry (Entitlement schema)

If you fool me, I will retaliate

Pull her into the van

I wanted to say that I had enough trouble on my mind.

I was sitting on the backseat with her

She is sitting next to me

Not angry anymore, maybe I felt good

Maybe I can get something from her, like a kiss, or something more

I asked her several times for a kiss

I wanted her to pay for making a fool out of me (that is what I thought)

She said no and kept saying no, meanwhile my friend drove away from the city, I told him, because it was quieter, finally we ended in a dark place.

She is struggling and saying no

Angry, callous, detached (Predator mode)

You do not reject me

I forced myself on the girl

I wanted her to pay for rejecting me.

I sexually abused her. 
Although he was very tense beforehand, Andy's presentation went well. He answered critical questions from the treatment team without becoming defensive. While it had been quite an emotional experience, the presentation provided Andy with a sense of closure regarding this difficult phase of treatment. Based on his presentation, Andy developed a relapse prevention plan together with his psychotherapist. Andy also gave a presentation to the team of psychiatric nurses on his ward. Subsequently, the treatment goals for the psychiatric nurses were formulated in schema mode language. The nurses received basic training in ST, and learned to recognize and respond to the patient's behaviors on the ward - for example, angry, impulsive, aggressive, avoidant, or dominant behavior - in terms of schema modes.

Limited reparenting, empathic confrontation and limit setting.

Limited reparenting, a focus on the patient's unmet emotional needs, was of central importance throughout Andy's psychotherapy, but especially in the initial phase, when the psychotherapist strives to establish an emotional bond with the patient. The most important basic needs for Andy were safety and stability, trustworthiness (e.g., keeping agreements), fairness and justice, consistency and clarity, transparency (e.g., admitting mistakes), and giving emotional support and attention. These basic needs of childhood are often not met in psychopathic patients (Chakhssi et al., 2012). Dr. TK adapted her limited reparenting stance to fit these basic needs. Being transparent, genuine, straightforward, and selfdisclosing within appropriate limits, were key aspects of her approach. An example of limited reparenting, after Andy has brought in a situation in which he had experienced unfairness that triggered his mistrust and abandonment schemas, is provided:

Andy: Last week, I had a quarrel with a nurse who treated me unfairly. He allowed another patient to stay in his room when he was feeling sick, but when I was feeling sick, I had to come to the dinner table that same evening! So I became angry and said: "This is not fair! Why do I have to come to the dinner table when I am feeling sick, and he doesn't?" Then I walked away and didn't talk to this nurse any more. He again confirmed that I can't trust him.

Dr. TK: I can imagine that you felt angry and mistreated, because you had to come to the dinner table and the other patient didn't while you were both not feeling well. I think this must feel the same for you as you felt in your childhood when you were constantly treated unfairly by your father. Children need their parents to be fair and consistent, because then they can grow up trusting other people. That's what you missed in your childhood.

Andy: Yes, you're right, he's just like my father. He treats me the same.

Dr. TK: I understand that this is painful for you. But on the other hand, Andy, I also want to be straight with you. You told me that you went to play soccer in the afternoon, so could it be possible that the nurse thought that you were feeling better and concluded that you could attend dinner at the table?

Andy: But then he should have asked me first! He didn't ask me at all if I was feeling better, he was only very authoritarian to me and you know: no-one can be authoritarian to me!

Dr. TK: So you missed his interest in you and in how you felt. You actually needed his attention and sympathy. 
Andy: Yes, that's what I felt, that no one cares about me as a human being, and they only see me as someone who doesn't comply with the rules on the ward.

Dr. TK: I understand, and I want to let you know that I care about you and that I really want to help you to handle these difficult situations and feelings. I will be there for you, at your side.

Dr. TK stated that she was affected by his vulnerability and the easily triggered feelings of being treated unfairly. It felt as if he was reliving an emotionally distressing experience belonging to a complex trauma. She indicated that he needed the reparenting because he never had someone who was there for him. Instead of dominating the therapy session and expressing his anger, Andy became silent, less tense, and listened to the therapist when she said she wanted to help him and to be there for him. Dr. TK also showed her caring towards Andy by occasionally acting on his behalf outside of sessions, for example, making phone calls when necessary, and meeting with the treatment coordinator and nurses of his ward to discuss issues concerning his treatment.

In the beginning, Andy tested if the Dr. TK would keep her agreements. He had always been strongly triggered if someone arrived late for an appointment, viewing this as evidence of untrustworthiness. On occasions when Dr. TK arrived late, he became very angry. After she let him ventilate his rage, they made a functional analysis of the situation. It became clear that the patient's sensitivity originated from the verbal and physical abuse he had suffered as a child, when his father arrived home late and exploded unpredictably in rage.

The psychotherapist made frequent use of empathic confrontation with regards to the effect of the modes on her, including self-disclosure (e.g., "I know that this is your protective side, but I feel threatened if you say....."). When she confronted the patient in this way, he was usually stunned, was able to stop his verbally aggressive behavior, and apologized. Through the ups and downs of psychotherapy, Dr. TK continued to offer reliability, openness and honesty.

ST makes extensive use of experiential techniques, such as chair work and guided imagery (Young et al., 2003), to reprocess the patient's emotional distress stemming from painful childhood experiences. At first, Andy refused the psychotherapist's suggestion to try experiential techniques. He found these exercises "artificial" and was afraid of losing control of his emotions. Dr. TK decided to wait until later in the psychotherapy to reintroduce these techniques.

Over time, Andy started to trust Dr. TK more, and a strong bond developed between them. He said he now noticed that his psychotherapist was a "human being" and not just a distant professional. Trust was central for Andy. Because he used to lie often, he was never trusted by others; now, he needed to feel that someone trusted and believed him. Obviously, Dr. TK realized that he might not be always telling the truth, but she decided to come from the position of trust rather than mistrust. Sometimes, Andy would take advantage of this trust, for example, by trying to use the material Dr. TK wrote in his treatment plan to his advantage. Dr. TK was careful not to write anything in her reports about the veracity of his accounts that she couldn't prove. If she felt that he was misusing her trust, she took more distance, and became business-like (e.g., "I said this, but not that"). Essentially, the Dr. TK's stance was: "I'll give you the benefit of the doubt, unless I'm proven otherwise. And if you do lie, then I want to understand why." In doing so, Dr. TK 
supported the healthy side of the patient, the side that wanted to be honest and forthcoming, and that was beginning to show genuine vulnerability. While in the first year his angry and impulsive sides were more prominent, during the second year, the vulnerable, abused child side became more visible.

Middle phase of therapy (second year, approximately).

Role of ancillary therapies (Milieu Therapy, Psychomotor Therapy) in treatment.

Over time, there was increasingly closer collaboration between Andy's ST psychotherapist and the psychiatric nurses on Andy's ward, as well as the other disciplines that were involved in his treatment. Dr. TK met regularly with the nurses, providing coaching about the mode approach that they were implementing on the ward (i.e., milieu therapy based on the schema mode model). Andy also began working with a movement therapist, who had received training in ST. The movement therapy focused on aggression and addiction issues, using experiential movement exercises to trigger schema modes, such as the bully and attack mode, so that the patient could learn to recognize and manage them. Through these exercises, Andy learned to recognize the signals in his body that tension was building up and how to intervene in a timely way, before he reached a point of no return. An example of a movement therapy technique was that Andy had to 'protect his territory' in the room. The goal for Andy was to recognize bodily tension in the bully and attack mode. The therapist's task was to enter his territory, to start a duel with the patient. During the first step, the therapist stopped when he observed Andy's arousal level became too high. During the second step, the therapist stopped after 1.5 minutes (indicated by the sound of a timer). During the third step, Andy had to stop himself when he felt his arousal became too high. Afterwards, Andy and the therapist watched the videotaped exercise so he could observe his body signals. He also recognized the overcompensatory nature of his aggression: because he had been physically abused himself, he had learned "to turn passive into active," becoming an aggressor himself.

Motivation and engagement in therapy, and therapeutic alliance.

Following his offense scenario presentation to the treatment team, Andy wanted to close the door on his past. Instead, Dr. TK pushed him gently but persistently to continue to examine difficult topics such as the abuse he suffered and to get in touch with his feelings. Over time, he realized that his need to keep his past at a distance was due to his mistrust and fear of losing control, and underneath it, painful feelings of vulnerability that he wanted to avoid. He continued to refuse to do experiential exercises, but agreed that further exploration of his modes could take place via role playing if he stayed in one chair, rather than use multiple chairs. Thus, the psychotherapist compromised, when necessary, to get the patient to try experiential work.

At about one and a half years into the treatment, Dr. TK noted that Andy's trust in her increased. He began to show real shame, became upset, and felt pain, when discussing his abuse history and his offense. Sometimes psychopathic patients find it easy to discuss their offenses, but not so with Andy, because his offense didn't fit his self-image. He felt shame for having violated a woman. In one session, he acknowledged that he was very anxious in sexual relationships, and had had trouble with sexual performance. He recalled his first sexual encounter when he was 12 years old with a girlfriend who was 14, when he was afraid to initiate sex. 
While he acted tough and macho with his antisocial friends, Andy felt quite anxious underneath. He also felt anxiety about intimacy when he later developed a relationship with a woman: "I had raped a woman. How would I feel when trying to initiate sex?"

Patient's behavior in the hospital.

During his second year of treatment, Andy showed increasingly more vulnerability and healthy adult behavior outside of ST. However, triggers continued to appear that left him feeling agitated, and made it difficult not to relapse into verbally aggressive behavior. A key moment occurred when his leave application was denied. This event triggered feelings of guilt and shame, and a self-punitive side became activated ("I am nothing, a monster, Hannibal Lecter"). He had suicidal thoughts, experienced considerable distress, and did not want to continue psychotherapy. Despite this setback, he was able to recover his equilibrium and move forward with his treatment, indicating growing resilience.

Andy started a new relationship with a young woman, an acquaintance of his mother. She often accompanied Andy's mother during her visits to the hospital. This led to discussions of his fears: "What is friendship? What is love? Is this a healthy relationship, when she has her freedom and I don't?" It also led to discussing previous relationships and what went wrong in them. Andy wanted to build this new relationship in a healthy adult way, without violence. The relationship lasted 6 months before he learned that his girlfriend was cheating on him. He broke off the relationship, recognizing that it was not a healthy one for him.

Final phase of therapy (last two years, approximately).

Experiential techniques: imaginary rescripting and letter writing.

Andy continued to refuse to do imagery exercises, which Dr. TK felt were essential to alleviating the emotional triggers that were at the root of his offenses. Eventually, after 2 years and 4 months of treatment, Dr. TK and his treatment coordinator decided to make his participation in these exercises a precondition for approval of his leave application. This limit setting succeeded: Andy finally agreed to do experiential exercises, although still with great reluctance. Andy and Dr. TK negotiated the conditions under which the imagery exercises would be conducted, which helped him to retain a measure of control. They agreed to try the exercises two or three times, and then evaluate how they were going before proceeding. Dr. TK also taught him to use the "time out" signal, so that he could end the exercise at any time. She began each exercise with a "safe place" image, where she asked Andy to close his eyes and imagine himself in a tranquil, safe environment.

In total, Andy and Dr. TK did five imagery exercises, all of which involved vivid recollections of physical and emotional abuse by his father. For example, in one exercise Andy clearly recalled a very hot day when he was 8- or 9-years old and his father locked him in a car for the whole day without food or water. In each of these exercises, the psychotherapist used imagery rescripting - that is, reworking the traumatic scene to give it a more satisfactory outcome - to counteract the destructive effects of these experiences. She asked Andy's permission to enter the image to protect the child and provide for his emotional needs. In the image, she confronted the father forcefully, standing up for the child and insisting that the father had no right to abuse him. She and Andy devised ways to protect the child, for example, having the police come to take the father to jail and lock him up. In a 
final imagery exercise, Andy recalled a later experience, when he was 16 years old, and was finally a physical match for his father. His father was beating him savagely, while Andy feigned a posture of passive surrender. At a certain moment, Andy caught his father off-guard, suddenly attacking him and beating him into submission. This was the last occasion on which his father had attempted to beat him. The image of turning the tables on his father made the overcompensatory nature of Andy's aggression understandable to him: by becoming a violent predator, he had learned how to avoid being a victim. After each of these exercises, Andy reported considerable relief, and feeling less easily triggered to anger and aggression in his interactions with others.

Later that year, Andy's supervised leave application was finally approved; under strict supervision of two guards and one psychiatric nurse, he was allowed to leave the hospital for periods of a few hours. Then, again, a stalemate occurred: he was no longer motivated to continue his treatment. He was eager to return to society and to put his past behind him. This reluctance to continue with treatment is a common reaction when patients begin the resocialization process and have a first "taste" of freedom. Eventually, his treatment coordinator had to set another limit: either Andy continued to work through his traumas and his offense or he would not write a new leave request. Andy agreed to continue his psychotherapy.

Dr. TK recommended that he write a letter to his father. At first, Andy refused, stating that he "wasn't a writer," and wanted to "stop delving into the past". However, Dr. TK and his treatment coordinator persisted and Andy eventually agreed, after 3.5 years of ST, to write the letter. A few quotes from the letter to his father read as follows::

\footnotetext{
"It pains me to write this. I was raised with a lot of violence in my home and I lived for 18 years in a completely unsafe environment. I have a lot of questions towards you: why so many beatings? So much violence? So much sadness? So many unsafe situations? So little love? So little warmth? So little family feeling?

A little child doesn't know right from wrong, you have to learn everything from your parents. I missed this at home, but I saw that it was more quiet and kind-hearted at my friends' home. I always thought about this and said to myself: this is the way I want to do it, when I have my own family.

I've always been angry towards you and I even made plans to kill you. I am really disappointed in you and I will never forget what you did to me, but I can learn from this and NEVER do this to my own family, but to guarantee them warmth, safety and the love they deserve. This will be the best revenge on you."
}

After the imagination exercises and writing the letter to his father, Andy reported feeling less pain about his past. He said that if he were to meet his father, he would be able to speak to him in a calm fashion. He would like to ask him: "Why have you beaten me so much? Why have you given me such a childhood?"

During this same period, Andy's relationship with his mother was discussed extensively. While warmer and less emotionally charged than his relationship with his father, Andy came to realize that he and his mother had an enmeshed relationship. In a sense, they were too close, which served his mother's emotional needs, but prevented him from becoming independent. Andy worked on breaking 
free from this enmeshment and began to develop more independence and sense of his own identity.

Reprocessing the offense.

Dr. TK wanted him to come to terms with his offense, taking responsibility for what he had done, processing feelings of shame and guilt, and then reaching a resolution in order to move on with his life. However, Andy was adamantly opposed to letting go of the pain and guilt that he experienced: he said that it reminded him of what he had done, and therefore served as a deterrent for the future. Eventually, Andy proposed a way to reprocess his offense. He decided to visit the scene of the offence.

In the meantime, he developed a new relationship with a young woman who visited him in the hospital. His new girlfriend had been sexually abused as a child, an issue that was very upsetting for Andy because of his own role as the perpetrator of a sexual offense. He discussed the sexual abuse and his own offense with his new girlfriend. His girlfriend said that she could forgive him for having raped a woman, which helped Andy forgive himself.

Resocialization phase of treatment.

The resocialization phase, in which Andy was allowed more frequent supervised leaves, and eventually, unsupervised leaves, was successful. As he earned more frequent leaves, he felt liberated and wanted to focus more on his life outside. The main triggers during this period were frustrations over the uncertainty and slowness of the resocialization process, and the continuation of the TBS-order. On the ward, Andy trusted the psychiatric nurses more, and was able to have more constructive discussions with them about his concerns. Thus, a generalization of learning was taking place. At first, only his psychotherapist and movement therapist could be trusted but by the end of treatment, he could discuss personal topics with some of the nurses, too.

Changes over the entire course of therapy.

A comprehensive evaluation of Andy's schema modes by Dr. TK showed that Andy had made significant progress. The antisocial modes, such as the predator, bully and attack, and self-aggrandizer modes, were clearly less prevalent than at the beginning. They were still present but less strong. Moreover, there was more room for healthy adult considerations and vulnerable emotions. His main vulnerability remained the mistrust/abuse schema (i.e., the expectation of being abused, mistreated or cheated by others). The triggers for mistrust remained inconsistent behavior or application of rules by authority figures, lack of clarity over rules, and unclear and inconsistent situations and communications in general. When Andy's mistrust was triggered, he continued to react either with avoidance ("I do not need them anymore") or with aggression. A positive change was that Andy could identify a trigger or conflict more quickly, and address it more constructively. For example, when he became mistrustful towards a male psychiatric nurse who had been recently assigned as his mentor ("He is not straightforward; he speaks with two tongues"), he addressed this immediately by arranging an appointment with the nurse to discuss his concerns. Also, in his new romantic relationship, he was noticeably less suspicious than previously. Andy acknowledged that abandonment fears in the relationship with his new girlfriend remained an important risk factor. At the same time, he recognized that even if the relationship were to end, he had 
learned a great deal from it and that it had helped him in his personal growth. Previously, Andy had always stated, "I engage in therapy for my mother, not for myself." At the end of this period, Andy said for the first time, "I engage in this process for myself, not for my girlfriend, and not for my mother."

Termination of therapy

After deliberation with Andy, Dr. TK and his treatment team decided to begin terminating ST. Over a period of about six months, the sessions were reduced to once every two or three weeks. Thereafter, sessions were even less frequent. Andy's relationship with his girlfriend continued to be monitored, as well as other aspects of his continuing reintegration into society. He took a position as a construction worker. The final 6 months of psychotherapy were focused on consolidating therapeutic gains and expanding his social and work network. Before his final session, Andy and Dr. TK watched a video recording of the initial phase of ST. Andy found it confronting to see himself as he previously was - how angry and aggressive he had been - which deepened his awareness of how much he had changed. Dr. TK gave Andy a card with a personal goodbye message. As requested by Dr. TK, Andy wrote the following evaluation in response to three questions:

\footnotetext{
How were you before the therapy?

“I behaved aggressively, trusted no one (was very suspicious), responded quickly and aggressively, let no one tell me what to do (especially not someone who was older than me) and was very rebellious and attributed everything to others."

What developments have you been through?

"I have learned to think differently. I am less worried about things that may happen to me or are happening. I have started to talk about my feelings and what's on my mind. That is my most important change."

How is it with your different sides (modes) at the moment?

"Some of the modes are gone (predator behavior and addiction) and some are still present, but to a lesser extent (trust/mistrust, powerful/powerless, impulsivity). They used to be problematic, but not anymore."
}

After he completed ST, Andy occasionally asked for follow-up sessions, especially when he became frustrated by the lack of progress in termination of his TBS-order. About one year after the end of ST, he was transferred to a resocialization ward and granted unsupervised leaves. Approximately one year later, after ending his second relationship during his TBS-order stay, he met another woman with whom he began a romantic relationship. At this time, he was living outside of the hospital. She soon became pregnant. When she gave birth, with Andy in attendance, the first person whom he thought of was his psychotherapist. She was the first person to whom he sent a birth-announcement.

Follow up interviews

About two years after ending ST, Dr. TK invited Andy for a follow up interview. Andy immediately agreed because he felt grateful and he "wanted to pay the psychotherapist back" for her efforts. During this interview, he told about his difficulties outside of the hospital, in getting a decent job, in the collaboration with his probation officer, and about the stigma of being a psychopath. He experienced life outside as much more difficult and there were more triggers than inside the hospital. He did not relapse into drug abuse or criminal behavior, although there 
were some situations that still triggered him (e.g., conflicts with his mother), though not to the same extent as before. An evaluation of the schema modes and triggers showed that the psychopathic modes were not present. When Dr. TK asked Andy about the meaning of ST and the therapeutic bond, he said he saw her as a real person who sometimes was touched by his pain, a mother figure, and that she had become a very important person in his life. Dr. TK was touched and surprised, especially as Andy had never before revealed such warm, personal feelings about her.

About three years after ending ST, Dr. TK again invited Andy for a follow up interview. This time, Andy seemed more adjusted to living in the community. He had a full-time job and provided for his girlfriend and their child. During the 3-year followup period he had not relapsed into drug abuse nor any criminal behavior, as confirmed by records obtained from the Department of Criminal Justice.

\section{Objective Assessment of Therapy Process and Outcomes}

To examine Andy's change from pre- to post-treatment, we used the reliable change index (RCl: Jacobson \& Truax, 1991). The RCl addresses whether a patient's change during treatment exceeds the change that is attributable to the standard measurement error of the instrument used. If the $\mathrm{RCl}$ is higher than 1.96, the probability that the pre-post treatment difference occurred by chance is less than $5 \%$. For the YSQ and the PCL-R, we used the reliability coefficients and standard deviations obtained in a comparable sample of personality disordered offenders to calculate the $\mathrm{RCl}$ (Chakhssi et al., 2012). For the BEST-Index, we used reliability coefficients and standard deviations from a BEST-Index validation study (Chakhssi et al., 2010a). Table 1 provides the mid- and/or post-treatment scores on the YSQ schema domains, BEST-Index total and scales scores, PCL-R total and facet scores, and the reliable change indices.

Psychopathy (PCL-R).

During the post-treatment PCL-R interview, Andy presented as a thoughtful person who had largely come to terms with his childhood experiences and the ensuing psychological pain. He also owned up to the offense that led to his conviction to the TBS-order. He was sincere, cooperative and willing to show his "weaker" sides. Andy obtained a PCL-R total score of 14 , which is clearly in the nonpsychopathic range. On the PCL-R Interpersonal facet, Andy obtained a score of 1 for his lies in the past and during the first phase of treatment. On the PCL-R Affective facet, Andy also scored 1: although he did show empathy towards the victim of the rape, he was less compassionate with the victim who went into coma after getting into a fight with him. On the PCL-R Impulsive Lifestyle facet, he obtained a score of 3 . Throughout his treatment in the forensic hospital, Andy showed behaviors that belonged to this facet. He still acted somewhat impulsively (mainly verbally) if he did not get his way. He showed responsible behavior during his hospital stay in terms of showing up for work-engagement- and therapeutic activities, and paying off a financial compensation for the victim. However, in the past, his irresponsible behaviors were extreme and he still had to show how he maintained his responsibility in the community. Finally on the PCL-R Antisocial facet, he scored 8 points. Although his behavioral controls improved substantially, he 
sometimes still acted out when he was frustrated. Most of the items belonging to the PCL-R Antisocial facet are historical and rated on the basis of previous (pretreatment) criminal behavior. As displayed in Table 3, the PCL-R total score showed significant improvement from pre- to post-treatment.

\section{TABLE 1}

Scores on the YSQ schema domains, BEST-Index and PCL-R Scores, and Reliable Change Indices for pretreatment to post-treatment

\begin{tabular}{lllll}
\hline Scales & Pre-treatment & Mid-treatment & Post-treatment & $\mathrm{RCl}$ \\
\hline Schema domains & & & & \\
Disconnection/Rejection & 2.91 & 1.55 & 1.65 & $6.59^{*}$ \\
Impaired Autonomy/Performance & 1.90 & 1.15 & 1.30 & $3.11^{*}$ \\
Impaired Limits & 3.58 & 1.41 & 1.97 & $5.06^{*}$ \\
Other-Directedness & 1.63 & 1.28 & 1.26 & 1.08 \\
Over-Vigilance/Inhibition & 3.44 & 1.59 & 1.47 & $5.44^{*}$ \\
BEST-Index Total & 266 & 275.5 & 306.5 & $4.76^{*}$ \\
Social Skills & 101.5 & 105 & 114.5 & $2.80^{*}$ \\
Insight & 82.5 & 87 & 101 & $3.74^{*}$ \\
Interpersonal Hostility & 50 & 48.5 & 56 & 1.49 \\
Physical Violence & 32 & 35 & 35 & 1.43 \\
PCL-R Total & 28 & NA & 14 & $3.2 *^{*}$ \\
Facet 1: Interpersonal & 4 & NA & 1 & 1.78 \\
Facet 2: Affective & 7 & NA & 1 & $4.74^{*}$ \\
Facet 3: Impulsive Lifestyle & 6 & NA & 3 & 1.63 \\
Facet 4: Antisocial & 8 & NA & 8 & 0
\end{tabular}

Note. $* p<.05 ; \mathrm{RCl}=$ Reliable Change Index; $\mathrm{NA}=$ Not Applicable; $\mathrm{RCl}$ scores for the schema domains and PCL-R have been reversed to correspond with positive treatment progress.

Early maladaptive schemas (YSQ).

The scores on the YSQ decreased significantly from pre- to post-treatment for four of the five EMS domains: Disconnection/Rejection, Impaired Autonomy /Performance, Impaired Limits and Overvigilance/Inhibition. The observed change on Other-directedness from pre- to post-treatment was not significant (see Table 1). This indicates that the majority of Andy's EMS have improved from pre- to posttreatment.

Risk-related behaviors (BEST-Index).

Andy's risk-related behaviors as measured with the BEST-Index showed a significant improvement from pre- to post-treatment on the total score, on Social Skills and Insight. The BEST-Index scale Social Skills measures adaptive social behavior, social skills, and the maintenance of interpersonal relationships. Based on the BEST-Index Social Skills scores, Andy developed better social skills and was better at maintaining relationships. The Insight scale measures insight into the 
nature of the one's problems and attribution of responsibility. The scale also measures how patients cope with stress. Based on the BEST-Index Insight scores, Andy developed more insight into his disorder and was able to more adequately cope with stressful events. According to the BEST-Index, Andy did not show significant improvement on Interpersonal Hostility and Physical Violence scores

Risk of future violence (HCR-20).

Andy's risk of future violence according to the HCR-20 decreased from "high" pre-treatment to "medium" post-treatment. According to the professional judgment of the assessor based on the HCR-20, Andy showed increased insight, fewer negative attitudes, less impulsivity and better treatment compliance. Also, Andy was judged to have become less susceptible to destabilizing events and more compliant with professional supervision.

The working alliance (WAI-O-S).

The scores on the WAI-O-S ranged between 4 and 5 , indicating that the coders observed positive indicators of the working alliance. The average total alliance score was $4.62(S D=.88), 4.95(S D=.89)$ for the Bond subscale, $4.25(S D=$ 1.03) for the Task subscale, and $4.65(S D=.97)$ for the Goal subscale. The average total alliance scores and subscales during the course of the psychotherapy are displayed in Table 2.

\section{TABLE 2}

Working alliance total and subscales scores (and SD) during the course of psychotherapy

\begin{tabular}{|c|c|c|c|c|}
\hline \multirow[b]{2}{*}{ WAI-O-S scores } & \multicolumn{4}{|c|}{ Time during psychotherapy } \\
\hline & $1^{\text {st }}$ year & $2^{\text {nd }}$ year & $3^{\text {rd }}$ year & $4^{\text {th }}$ year \\
\hline Total & $4.11(.06)$ & $4.83(.38)$ & $4.79(.40)$ & $4.72(.53)$ \\
\hline Bond & $4.50(.42)$ & $5.29(.13)$ & $4.96(.38)$ & $5.04(.63)$ \\
\hline Task & $3.67(.08)$ & $4.50(.05)$ & $4.54(.45)$ & $4.29(.46)$ \\
\hline Goal & $4.17(.17)$ & $4.71(.54)$ & $4.88(.38)$ & $4.83(.50)$ \\
\hline
\end{tabular}

Note. WAI-O-S = Working Alliance Inventory - Observer, Short version.

Scores on working alliance for patients with psychopathic traits have not been reported in the literature, although some scholars suggest that psychopathic patients are unable to form a therapeutic bond (Skeem et al., 2002). However, the average alliance ratings for this patient were indicative of a positive working alliance and comparable to average alliance ratings of therapists and patients in a sample of borderline outpatients who completed treatment (Spinhoven, Giesen-Bloo, van Dyck, Kooiman, \& Arntz, 2007), higher than therapist alliance ratings of borderline outpatients who dropped out of treatment (Spinhoven et al., 2007), and also higher 
than the average alliance ratings with the WAI-O (long version) in a sample of substance abusers (Fenton, Cecero, Nich, Frankforter, \& Carroll, 2001).

\section{DISCUSSION}

This single case study is the first to describe the apparently effective treatment of a psychopathic patient. The treatment included individual ST, combined with movement therapy and support from the nurse staff. After the 4-year treatment and 3-year follow-up, the patient no longer displayed prominent psychopathic features, displaying more empathy, shame, and guilt, and significantly more insight and better communication skills compared to his pre-treatment scores. Although he remained somewhat mistrustful, he was able to develop meaningful interpersonal and intimate relationships: he became a father, took adequate care of his family and maintained a job. He reprocessed painful feelings from childhood experiences with his father and developed more autonomy in his relationship with his mother. Although the treatment posed many challenges, this case study refutes the widely held view that patients with psychopathic features are untreatable, or that treatment makes them worse (Rice et al., 1992; Seto \& Barbaree, 1999). In contrast to clinical lore, the patient was able to engage in treatment (e.g., Hobson, Shine, \& Roberts, 2000; Ogloff, Wong, \& Greenwood, 1990), form a bond with his therapist (e.g., Lösel, 1995), and show a positive treatment response. Moreover, the treatment did not appear to make the patient more dangerous (e.g., Rice et al., 1992; Seto \& Barbaree, 1999). These findings concur with those of recent studies indicating that some psychopathic patients may benefit from treatment (Chakhssi et al., 2010a; Hildebrand \& de Ruiter, 2012; Olver \& Wong, 2009; Skeem et al., 2002). They are also consistent with preliminary, but not statistically significant, results from a randomized clinical trial in the Netherlands suggesting that ST reduces risk and speeds re-entry into the community in forensic patients with Cluster B personality disorders, including psychopathic ones (Bernstein et al., 2012).

In contrast to standard cognitive behavioral therapy, ST places more emphasis on the childhood origins of maladaptive behaviors, and uses the therapeutic relationship (e.g., limited reparenting, empathic confrontation) and experiential, emotion-focused techniques to reach the patient's vulnerable side (Rafaeli et al., 2011; Young et al., 2003). This may also explain the prolonged time, nearly a year, it took to develop an integrated case conceptualization in Andy's case, including an offense scenario analysis. Although this was the psychotherapist's first case using ST with a patient with psychopathic traits, and future case conceptualizations may require less time, we believe that developing an integrated case conceptualization whilst focusing on the therapeutic alliance is essential in the treatment of patients with psychopathic traits and may require more time than usual (5 to 8 sessions). This case study showed that it was possible to break through the emotional detachment of a psychopathic patient to heal his underlying emotional pain, reflected in significantly improved scores in four of the five EMS domains. This is consistent with a recent pilot study showing that ST was about twice as effective treatment-as-usual in reaching forensic patients' vulnerable emotions (Van den Broek, Keulen-de Vos, \& Bernstein, 2011). 
One of the premises of ST is that individuals are capable of experiencing at least a minimum of empathy, emotions and feelings of connectedness. This case study showed that a patient's potential for empathy and attachment may not be evident at the beginning of treatment. At the start, Andy was described by some of those who worked with him as a "classic psychopath". Furthermore, in our clinical experience, there are patients who appear to possess callous-unemotional traits and a history of childhood trauma, and he callousness may serve as a protection against experiencing vulnerable emotions. However, until there are sufficient empirical data on which patients are likely to benefit from ST, and which are not, it is premature to exclude patients from ST because certain psychopathic features, such as callous-unemotional traits, are present.

By the end of his treatment and follow-up, Andy's scores on PCL-R items for prominent psychopathic features such as lack of empathy, impulsivity, and failure to take responsibility, were reduced from high to moderate, indicating improvement on these characteristics, but a certain degree of risk of future violence still remained. Andy's scores on the HCR-20 risk assessment instrument were also reduced from high to medium. Thus, while our best estimate is that Andy's risk for future violence has diminished, we cannot say with certainty that he will not recidivate. ST makes no claims to completely eliminate risk in psychopathic or other personality disordered patients (Bernstein et al., 2007; Bernstein et al., 2012). Follow-up in the future will determine whether the gains made will hold up while living in the community. Certainly, his ability to exert self-control over his behavior during his final treatment phase offers reason to be hopeful about the future.

The findings of our study have several limitations. First, Andy's pre-treatment PCL-R score of 28.4 was slightly lower than the conventional cutoff score of 30 used in North-American samples (i.e., PCL-R total score > 30), but was higher than the cutoff score of 25 used in European samples (Cooke, Michie, Hart, \& Clark, 2005; Hare, 2003). Also, it was higher than the cut-off score (PCL-R > 25) used in studies that demonstrate that psychopathy is a more severe form of ASPD than ASPD alone (e.g., Coid \& Ullrich, 2010). However, it may be difficult to generalize these results to patients with much higher PCL-R scores, such as those with scores above 30. Furthermore, the length of treatment in Andy's case was 4 years, which was longer than the 3 years applied in the recent clinical trials of ST in BPD patients (Giesen-Bloo et al., 2006; Farrell et al., 2009), but shorter than the average time until conditional discharge ( $\mathrm{M}=9.8$ years) in Dutch TBS-patients (Nagtegaal, van der Horst, \& Schönberger, 2011).

Finally, psychopathic patients are well-versed in impression management (e.g., Lilienfeld, 1994). We cannot verify with certainty the patient's reports of the motives for his offense, nor his accounts of his life after leaving the institution. Thus, we cannot be absolutely sure that the patient's treatment progress was genuine and not a manipulative attempt to shorten his forensic psychiatric treatment. On the other hand, there is also evidence of the patient's apparently sincere efforts to come to terms with his offenses and refrain from further antisocial behavior. The improvements on the standardized assessment instruments also support this positive view. Note that three out of the four instruments were observer-rated tools, rated during the course of treatment by several different and independent raters, indicating that positive progress was not 
confined to the therapy sessions. This positive view is also supported by the absence of recidivism according to official arrest records. Recidivism rates for psychopaths are high: about three to four times higher than for other offenders at 1 to 3 years after discharge from secure institutions (Hemphill, Hare, \& Wong, 1998). In this light, Andy's lack of reoffending over a period of 3 years post release is reassuring. Thus, while some ambiguities remain, our overall impression is of a patient whose progress is genuine.

\section{Future directions}

While many questions about the nature of change in psychopathic patients remain, we conclude that some or perhaps even many of these patients may prove amenable to treatment, if they are given an evidence-supported therapy that is specifically targeted to the nature of their problems. More research is needed into a variety of treatment methods to further investigate which patients with psychopathic traits are able to benefit from which treatment method, or a combination thereof. A randomized clinical trial to test the hypothesis that ST is an effective treatment for personality disordered offenders, including psychopathic ones, is currently in progress (Bernstein et al., 2012). 
110 | CHAPTER 6 
General Discussion

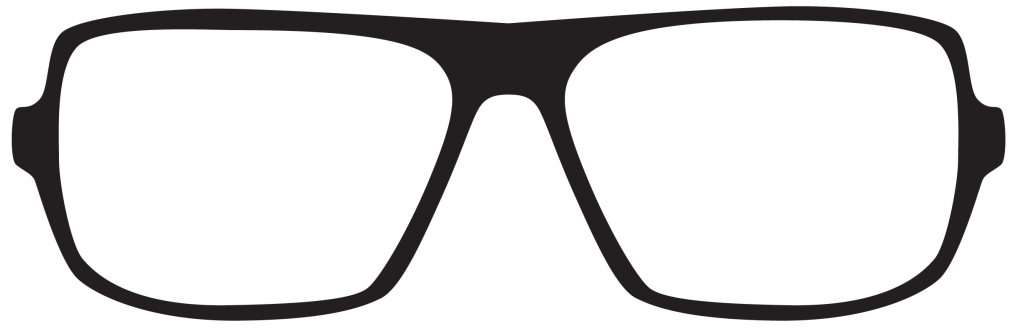


Psychopathy as measured with the Psychopathy Checklist-Revised (PCL-R; Hare, $1991 ; 2003)$ is considered to be among the most important constructs in forensic psychiatry. Psychopaths are three to five times more likely to reoffend than nonpsychopaths (Hemphill, Hare, \& Wong, 1998; Leistico, Salekin, DeCoster, \& Rogers, 2008), and prevalence rates between $24 \%$ and $35 \%$ have been reported in Dutch forensic psychiatric populations (Hildebrand, Hesper, Spreen, \& Nijman, 2005; Hildebrand \& de Ruiter, 2004). Many clinicians and researchers assume that psychopathy is untreatable (Salekin, 2002), a view that has led to the idea to exclude psychopaths from forensic treatment (e.g., Commissie Visser, 2006). However, perhaps because of the entrenched therapeutic pessimism about psychopathy, very few empirical studies have actually examined psychopaths' treatment responsiveness, especially in Dutch forensic psychiatry.

The research reported in this thesis was aimed at shedding light on the treatment responsiveness of psychopaths and examining the contribution of schema therapy concepts and interventions in psychopaths and other offenders. In this final chapter, the most important findings of the research are summarized and several limitations and strengths are discussed. Furthermore, suggestions for future research and implications for clinical practice are provided.

\section{Main empirical findings}

\section{Part I: Assessment}

\section{Measuring change}

Commonly used measures (i.e., self-report methods) to assess change during treatment may be subject to impression management (i.e., faking good) in forensic populations. Well-validated instruments for measuring change during forensic treatment that rely on other information sources, such as observations of riskrelated behaviors, are scarce. Hence, the first aim of this thesis was to examine a broad-spectrum observational measure for violence risk-related indicators as a possible instrument for measuring change during forensic treatment. In Chapter 2, the reliability and validity of a nurse-rated forensic assessment tool, the Behavioural Status Index (BEST-Index; Woods, Reed, \& Robinson, 1999), was examined. This thesis contains the first study into the psychometric properties of the Dutch BESTIndex, which revealed a different and clearer factor structure compared to the original one (Woods et al., 1999). The revised version of the BEST-Index was found to be an internally consistent measure that can be reliably rated by paraprofessionals in order to assess potentially risk-relevant behavior among forensic psychiatric inpatients. Furthermore, the revised BEST-Index was significantly related to future violence risk as measured with the Historical Clinical Risk Management-20 (HCR-20; Webster, Douglas, Eaves, \& Hart, 1997) and to aggressive behavior in the hospital. Our findings revealed that the revised BESTIndex predicted inpatient aggressive behavior as well as, if not better than, the HCR20. Specifically, the revised BEST-Index factor Interpersonal hostility showed stronger associations with various inpatient aggressive behaviors than the HCR-20 (see Chapter 2). Our findings concur with international findings regarding the 
interrater reliability and the convergent validity of the BEST-Index (Ross et al., 2008; Woods et al., 1999).

\section{Early maladaptive schemas}

We examined the relationship between Early Maladaptive Schemas (EMS), PCL$\mathrm{R}$ facets, and institutional violence in a sample of violent offenders with personality disorders. To our knowledge, no study had previously examined EMS in violent offenders with personality disorders and/or psychopathic traits.

In our sample, the association between EMS and the PCL-R facets were consistent with Beck et al.'s (1990) hypotheses on maladaptive cognitions in highly antisocial individuals (see Chapter 3). More specifically, a history of an impulsive lifestyle and persistent antisocial behavior, according to the PCL-R, was significantly associated with EMS related to a high level of mistrust and a hostile view of the world in terms of predators and prey, to inadequate self-control and low frustration tolerance, and to a strong need for autonomy and dominance. Although EMS have not been previously examined in violent personality disordered offenders, these findings appear to fit with the antisocial, interpersonal, and/or impulsive lifestyle features of psychopathy. This may indicate that EMS, presumably developed early in life as a consequence of childhood adversity, may play a role in several psychopathic traits in adulthood. Furthermore, the PCL-R Antisocial facet was the only PCL-R facet significantly associated with inpatient violence during a follow-up period of two years. This finding is consistent with the current literature regarding the predictive validity of the PCL-R for institutional violence in forensic patients (e.g., Walters \& Heilbrun, 2010). Contrary to our expectations, the hypothesized EMS were not related to inpatient violence in our sample.

To explain this finding, we considered Young et al.'s (2003) schema theory, in which externalizing behaviors (e.g., verbal or physical aggression) are called schema modes and are considered maladaptive coping responses to EMS and their emotional state-related manifestations. Previous studies have shown that schema modes may account for the rapid changes in mood and behavior observed in personality disordered patients when EMS are triggered in specific situations (Lobbestael \& Arntz, 2010). For example, antisocial patients may "switch" between states of vigilance and aggression, depending on the intensity and/or imminence of the anger triggering event (Lobbestael, Arntz, Cima, \& Chakhssi, 2009). This is more likely when the anger-triggering event is related to past experiences of conflict and/or other previous anger-inducing events (Lobbestael \& Arntz, 2012). In the hospital setting where the present study was conducted, the intensity and/or imminence of the anger-triggering events may not always have passed the threshold for the aggressive schema mode as a result of preventive risk management strategies carried out by the mental health professionals. Another reason for the lack of association between EMS and institutional violence may be that we did not differentiate in types of violence (e.g., reactive vs. instrumental). The theory of EMS suggests that reactive aggression may be more associated to EMS as a maladaptive and emotionally reactive response to the triggered EMS and the subsequent experience of strong negative emotions, such as shame, fear, or rage.

Next, we examined early maladaptive schemas and psychopathy in male child sexual offenders and compared them with male sexual offenders against adults and 
male nonsexually violent offenders. When controlling for degree of psychopathy, child sexual offenders were more likely than nonsexually violent offenders to have EMS related to feelings of loneliness, lack of intimacy, lack of self-esteem and problematic interpersonal relationships (see Chapter 4). Sexual offenders against adults were more likely than nonsexually violent offenders to have EMS related to problematic interpersonal relationships. Consistent with the literature, these findings suggests that the sequelae of childhood adversity may be related to sexual offending in adulthood (Salter et al., 2003). In addition to sexual deviance, the schematic structure related to social isolation, intimacy deficits, problematic interpersonal relationships and lack of self-esteem have been regarded as key issues in the treatment programs of sexual offenders, including child sexual offenders (Marshall, Marshall, Serran, \& O’Brien, 2011). Also, some evidence exists that targeting these areas in cognitive-behavioral treatment may decrease sexual recidivism in (child) sexual offenders (Lösel \& Schmucker, 2005).

Taken together, the prevalence of EMS in our study sample suggests that EMS theory may inform the forensic assessment and treatment of violent offenders with psychopathic traits, including (child) sexual offenders. The theory of EMS offers a more intuitive approach for mental health professionals by providing a rationale linking historical antecedents to present maladaptive cognitions and behavior.

\section{Part II: Treatment}

Change in psychopaths versus nonpsychopaths

In Chapter 5, we examined change during long-term inpatient forensic treatment in psychopathic and nonpsychopathic offenders. In contrast to clinical lore, our findings suggest that forensic treatment does not make all or most psychopaths worse. Group- and individual-level analyses showed no significant differences in change during treatment between psychopathic and nonpsychopathic patients on a number of risk-related indicators: adaptive social behavior, communication skills, insight, attribution of responsibility and self-regulation strategies. Also, the majority of psychopaths in our sample showed little or no physical aggression or interpersonally hostile behavior during the course of their treatment (20-months), suggesting that forensic treatment may help them to exert better self-control over their behavior. However, psychopaths did show less change on the risk indicator of interpersonally hostile behavior compared to nonpsychopaths, and a subgroup of psychopaths (22\%) deteriorated during treatment with regard to degree of physical aggression, whereas none of the nonpsychopaths did. The findings that psychopaths are more likely to show aggression than nonpsychopaths during hospitalization is consistent with previous research (e.g., Hildebrand, de Ruiter, \& Nijman, 2004; Hobson, Shine, \& Roberts, 2000; Ogloff, Wong, \& Greenwood, 1990). To explain these findings, we considered the results of brain imaging studies in psychopaths (e.g., Raine, Lencz, Bihrle, LaCasse, \& Colletti, 2000), in that psychopaths who deteriorated during forensic treatment with regard to aggressive behaviors may have poorer functioning in brain regions (e.g., the prefrontal cortex) involved in regulating and controlling aggression. An additional explanation could be that these deteriorating psychopaths may have a more serious history of repeated maladaptive, impulsive behaviors and persistent 
antisocial behaviors and probably developed more serious EMS during the course of their life, which require more intensive and longer-term forensic treatment than was offered in the current study.

Although these findings contradict the widely held belief that psychopathy is immutable (e.g., Cleckley, 1988; Kernberg, Yeomans, Clarkin, \& Levy, 2008; Reid \& Gacono, 2000), change in psychopathic traits themselves was not examined. Studies addressing the treatment response of a particular disorder, in this case psychopathy, should also examine change in the core characteristics of that disorder (see Chapter 6). Also, the long-term outcomes of the study sample will have to be examined to determine the course of these changes after hospitalization. In terms of treatment effects on recidivism, a growing body of research suggests that more optimism concerning the effects on violent recidivism of psychological treatment for psychopathic patients is warranted (see Salekin, Worley, \& Grimes, 2010).

\section{Schema Therapy of a psychopathic patient}

In Chapter 6, we examined long-term schema therapy (ST) of a forensic psychiatric patient with psychopathic traits. The findings of the single case study provided a detailed clinical description of the therapy process and showed that ST can achieve significant positive changes in psychopathic traits, cognitive schemas and risk-related behaviors in a male forensic patient sentenced to mandatory treatment. The 3-year follow-up during the patient's conditional release from the forensic hospital showed that the patient was able to desist from impulsive or aggressive behavior, integrate into the community and provide for his girlfriend and newborn child without relapsing into criminal offending.

The case study showed that ST could be applied to the treatment of psychopathic traits with some adaptations compared to ST for personality disorders more generally (Young, Klosko, \& Weishaar, 2003). First, the ST case conceptualization was expanded by incorporating the index offense. The assessment of EMS and schema modes, their historical antecedents and resulting present problems were linked to the offender's specific thoughts, feelings, behaviors and high-risk situations that played a role in his offense. Incorporating the index offense in the ST case conceptualization required a longer period of time, in this case one year and three months, than normally is the case (i.e., five to six sessions). As a consequence, the length of the therapy exceeded the three years mostly applied in clinical trials of ST in severe personality disordered patients by one and half year (Farrell, Shaw, \& Webber, 2009; Giesen-Bloo et al., 2006). A second adaptation was expanding the original schema mode model (Young et al., 2003) with schema modes relevant for criminal and aggressive behavior (Bernstein, Arntz, \& de Vos, 2007). Third, another adaptation compared to regular ST was that the ST case conceptualization was shared beyond the confidentiality of the therapy sessions. It was shared with the milieu treatment staff involved, including psychiatric nurses on the ward, and with the patient's social network to provide an environment where several mental health professionals, with the support of the patient's network were working towards mutual treatment goals as well as to monitor generalization of learning outside the therapy sessions. 


\section{Strengths and limitations}

\section{Strengths}

The main strength of this thesis is that all studies were conducted in a forensic clinical setting. First, the assessments of the PCL-R, HCR-20, and BEST-Index were performed by clinicians and forensic psychiatric nurses as part of their clinical routine. The findings in this thesis were based on "real world" use of the measures, and the field reliability of the measures was comparable to the reliability achieved by researchers (e.g., Hare, 2003; Hildebrand et al., 2005), and thus contribute to the ecological validity of our findings. Reliability studies suggest that the field reliability of the PCL-R, and perhaps other measures used for risk assessment, is often lower than the research reliability reported in the instruments' manuals or in controlled studies (Edens, Boccaccini, \& Johnson, 2010; Miller, Rufino, Boccaccini, Jackson, \& Murrie, 2011; Murrie, Boccaccini, Johnson, \& Janke, 2008; Murrie et al., 2009).

Second, the prospective studies were based on observational data obtained longitudinally during forensic treatment and assessed by different raters. We used an observational measure of change which was less susceptible to response bias, such as socially desirable responding, which often affects the responses of forensic patients to self-report questions (e.g., Cima et al., 2003; Lilienfeld, 1994). Most other studies regarding treatment response in psychopaths were retrospective in nature and/or used ratings by researchers (e.g., Ogloff et al., 1990; Olver \& Wong, 2009; Rice, Harris, \& Cormier, 1992; Skeem, Monahan, \& Mulvey 2002).

Another strength was that we used the Dutch version of the PCL-R to assess psychopathy - instead of, for instance, a self-report measure of psychopathy because the PCL-R was specifically developed to assess psychopathy in forensic and correctional settings, and is the most extensively validated measure of psychopathy (Skeem, Polaschek, Patrick, \& Lilienfeld, 2011). The Dutch version of the PCL-R (Vertommen, Verheul, de Ruiter, \& Hildebrand, 2002) has been adapted to acknowledge the specific jurisdiction in the Netherlands, and has been validated in Dutch forensic psychiatric samples (Hildebrand, 2004). It is noteworthy that our study that examined change in psychopaths during forensic treatment, to date, is one of just two studies examining PCL-R psychopathy in relation to treatment response in Dutch forensic psychiatry (see Hildebrand \& de Ruiter, 2012). Internationally, Salekin, Worley, and Grimes (2010) identified only eight studies in the literature examining treatment response in adult psychopaths using the PCL-R, and in a previous meta-analysis examining treatment response in psychopaths only four out of 42 studies used the PCL-R to assess psychopathy (Salekin, 2002).

In addition to group-level analyses, we investigated change during treatment in psychopathic patients on an individual level. Our findings showed that within the group of psychopaths, one third showed reliable improvement. The finding that some psychopaths improve during treatment would have been masked if the data were analyzed solely on a group level.

Finally, we used a general theory of psychopathology, in this case Young's schema therapy model (Young et al., 2003) and its adaptation for forensic populations (Bernstein et al., 2007), to further our understanding of psychopathy as a personality disorder. This is an innovative approach in the contemporary literature 
where psychopathy has been chiefly conceptualized as an inherently antisocial condition instead of a psychological disturbance (Newman, 1998), and in some instances as a manifestation of pure "evil" (e.g., Tangney \& Stuewig, 2004). Indeed, the mere use of the label psychopath for an individual implies a judgment as more dangerous and untreatable, resulting in denial of mental health services and eliciting strong support for harsher punishments compared to nonpsychopathic offenders who have committed similar crimes (Boccaccini, Murrie, Clark, \& Cornell, 2008; Cavadino, 1998; Edens, Colwell, Desforges, \& Fernandez, 2005). Adjacent to this issue, is that reframing psychopathy using schema therapy takes the influence of early traumatic experiences and the development of psychopathology into account. Not acknowledging the influence of the adverse experiences an individual with psychopathic features has been exposed to, and not recognizing their influence on his current behavior, may be a repetition of their earlier trauma, such as abuse, neglect and conditional acceptance, and may thus perpetuate maladaptive traits and coping responses. In a way it is inherent to the PCL-R not to acknowledge the possible developmental background of psychopathic traits. Thus, utilizing a more general approach, looking beyond the pejorative label of psychopathy, and acknowledging a patient traits from a developmental psychopathology perspective, may enhance motivation for and responsivity to forensic treatment, and might make treatment for this population more effective.

\section{Limitations}

All of the studies in the thesis involved male forensic psychiatric patients with personality disorders and/or psychopathic traits from one Dutch forensic psychiatric hospital, and several limitations should be considered to properly interpret the findings of this thesis.

The generalizability of the findings may not extend to other inpatient male forensic psychiatric patients with personality disorders or psychopathic traits. We have not been able to compare the characteristics of our sample with patients from other Dutch forensic psychiatric hospitals. However, since the start of the hospital in 2000, offenders with a TBS order have been randomly assigned to any forensic hospital, with the exception of borderline intellectual functioning offenders (i.e., low IQ), female offenders and offenders who are extremely difficult to manage and pose a high flight risk (Commissie Visser, 2006). Our samples did not include female offenders and we excluded offenders with borderline intellectual functioning in most studies. Nevertheless, our findings from the study examining treatment responsiveness in psychopaths were similar to the findings examining treatment responsiveness in psychopaths in another Dutch forensic hospital (Hildebrand \& de Ruiter, 2012). Thus, we believe that our sample represents Dutch forensic psychiatric inpatients with personality disorders or psychopathic traits.

Another limitation of the current thesis is the lack of a no-treatment control group or non-offender control group. However, the lack of no treatment control group is inherent to forensic clinical outcome studies where withholding treatment is seen as unwarranted and/or unethical. The lack of a non-offending control group in the studies examining EMS in personality disordered offenders leaves open the possibility that the schema structures found (see Chapter 3 and 4) may not be specific for offenders with personality disorders and/or psychopathic traits. 
The cross-sectional design of the prevalence study of EMS in offenders' studies is also a limitation. The significant correlations do not imply directionality and strong evidence that EMS develop in early childhood is absent. The relationship between adverse childhood experiences and EMS is theoretically assumed, but not empirically validated. One could also argue that personality disturbances may lead to the development of EMS, instead of Young et al.'s (2003) hypothesis that EMS are risk factors for the development of personality disorders. Also, the findings that specific EMS are prevalent in sexual offenders (see Chapter 4) may also be explained as post-hoc rationalization of their sexual offences. Another limitation was the small sample sizes in the studies examining EMS in sexual offenders, which may, to some extent, have limited the power to detect further differences in EMS between the offender groups.

Finally, DSM-IV-TR classifications of the samples were gathered from hospital files. DSM-IV-TR Axis-II diagnoses were based on the Structured Interview for DSM-IV Personality Disorders (SIDP-IV; Pfohl, Blum, \& Zimmerman, 1997). Although, the DSM-IV-TR Axis-I classifications were primarily analyzed as descriptives, their diagnostic accuracy remains unknown.

\section{Clinical and Policy Implications}

The demonstrated treatment responsiveness of psychopaths compared to nonpsychopaths does not support the policy to withhold forensic treatment from psychopaths. Instead, the observed change during forensic treatment offers more optimism in examining possible innovative treatment methods for psychopathic patients, with the goal of reducing their risk of recidivism. Based on the findings of this thesis, one of the innovative treatment methods worth exploring is schema therapy (Young et al., 2003), and the adaptation for forensic populations by Bernstein and colleagues (2007). Also, the findings in this thesis that some psychopaths continue to display aggressive and hostile behaviors during institutional treatment, suggests that supplemental assessment and treatment methods may be required. For example, pharmacological therapy adjacent to psychological treatment interventions that target mistrust, insufficient self-control and dominance (e.g., re-processing trauma, reducing externalizing maladaptive coping, improving distress tolerance, and problem solving) may be particularly relevant to reducing aggression in some psychopathic patients.

Our findings also stress the importance to adequately assess the cognitive sequelae of adverse childhood experiences in offenders with personality disorders and/or psychopathic traits. Assessment of adverse experiences during childhood is common clinical practice within forensic psychiatry, but rarely structured. The prevalence of EMS in offenders should lead forensic mental health professionals to use a more structured approach in examining which adverse experiences a patient has encountered and how these experiences are related to his present maladaptive behaviors and dynamic (i.e., changeable) risk factors. For example, using structured approaches to assess adverse experiences, such as the Childhood Trauma Questionnaire (Bernstein \& Fink, 1998) or the Interview for Traumatic Events in Childhood (Lobbestael, Arntz, Harkema-Schouten, \& Bernstein, 2009) together with structured approaches for assessing violence risk, such as the HCR-20 (Webster et al., 1997) may aid mental health professionals to serve this purpose. Also, the use 
of the Young Schema Questionnaire (Sterk \& Rijkeboer, 1997), and the Schema Mode Inventory (Lobbestael, van Vreeswijk, Spinhoven, Schouten, \& Arntz, 2010) may further help therapists and patients find a rationale for understanding their offending behavior and a theoretical framework on which treatment interventions could be based.

The validation study of the BEST-Index provides Dutch forensic clinical practice with an additional reliable tool for measuring change in risk-related behaviors during forensic treatment. Furthermore, the BEST-Index showed significant associations with the HCR-20 and inpatient violence, which supports its validity. The BEST-Index focuses primarily on assessing changeable risk-related behaviors, and may fill the increasing needs for instruments, in addition to risk assessment instruments, to measure change in risk level during forensic psychiatric treatment.

Our findings also have implications for existing policy regulations. The first implication concerns the "long stay" facilities that have been implemented within Dutch forensic psychiatry in the last decade. These facilities are intended for patients who are deemed untreatable, are considered "chronically dangerous", and require long-term risk management (de Kogel, Verwers, \& den Hartogh, 2005). Patients admitted to long stay facilities are no longer treated with the aim of resocialization into the community and this regulation has been criticized as concealed life imprisonment (Raad voor Strafrechttoepassing en Jeugdbescherming, 2008). Although the admission criteria concerning untreatability and chronic dangerousness are ill-defined (Hildebrand \& de Ruiter, 2010), psychopathy is explicitly mentioned as a "disability" interfering with treatment amenability (Ministerie van Justitie, 2009). The research in this thesis, and the accumulating evidence in the current empirical literature, does not support psychopathy as a diagnosis equated with untreatability. Psychopathy in itself represents a disorder and like all other disorders, in the absence of sufficient evidence concerning its treatability, continued treatment efforts and further research into effective treatment strategies are warranted.

The second implication concerns the policy surrounding leave privileges in Dutch forensic psychiatry. Leave privileges are regarded as an essential part of forensic treatment and a prerequisite for successful resocialization into the community. However, PCL-R raw scores have a prominent place in leave applications and are decisive in granting leave privileges by the Department of Justice (Adviescollege Verloftoetsing tbs [AVT], 2012). This is most pertinent for patients with PCL-R scores above 26, for whom the mere lack of an extensive description of the PCL-R facets in their application is sufficient reason to deny them leave privileges (AVT, 2012), independent of their treatment gains, inpatient behavior and scores on other instruments. This is troublesome for two major reasons. The first reason is the adverse effect denying leave privileges, solely based on the label psychopath, has on the commitment and motivation for treatment of these patients. Illustrative to this point is the case study (see Chapter 6) where denial of the leave privilege resulted in the patient experiencing considerable distress, emotional detachment and refusal to continue therapy. Although forensic treatment is aimed at teaching patients to cope with distress without resorting to antisocial behavior, introducing distress unnecessarily could be considered unethical. The second reason is the use of the PCL-R raw scores and 
the issue of measurement error together with the lower reliability of the PCL-R in clinical practice (e.g., Edens et al., 2010). According to the PCL-R Manual (Hare, 2003), the measurement error of the PCL-R is approximately 3 points, which is mostly based on studies conducted by researchers. Thus, under the optimal circumstance that field reliability reaches the same level as the reliabilities in the PCL-R manual, an individual PCL-R score ranges minus or plus 6 points within a 95 percent confidence interval. For example, chances are 95 out of 100 that when a patient obtains a PCL-R total score of 24 , his "true" score will fall between 18 and 30. Therefore, it has been long recommend that the label of psychopath, and the use of PCL-R raw scores, should be replaced in correctional and forensic settings with a percentile rank on the PCL-R (Hare, 1998). Similarly, the Dutch Association of Psychologists (2010) recommend in their guidelines for psychological assessment that evaluators should report confidence intervals for tests where raw scores may (unintentionally) lead to improper use by (nonpsychologist) consumers of such evaluations.

\section{Suggestions for further research}

- $\quad$ More research is needed examining the treatment responsiveness of individuals with psychopathic traits and long-term follow up after they have been released into the community.

- More research is needed into a variety of treatment methods to further investigate which offenders with psychopathic traits are able to benefit from which treatment method, or a combination thereof. Currently, a randomized clinical trial is underway (Bernstein et al., 2012) examining if ST may be an effective treatment for personality disordered offenders, including psychopathic ones.

- $\quad$ To support to use of ST for offenders with personality disorder and/or psychopathic traits, more research is needed relating changes in EMS and schema modes to changes in personality disorder traits, psychopathic traits and risk of future violence.

- More empirical evidence for the prevalence of EMS in (sexual) offenders, and for the association between EMS and impulsive lifestyle and antisocial behaviors is needed. For example, by replicating the present studies in larger and different samples (e.g., outpatient- and correctional samples).

- The theoretical assumptions underlying the relevance of EMS in relation to aggression in violent offenders needs more research. Future research should examine whether EMS show differential relationships with reactive and/or instrumental violence.

- Finally, the predictive validity of the BEST-Index for desistance from criminal behavior after discharge from the forensic hospital should be investigated, thereby offering empirical evidence for the relevance of change on riskrelated behaviors during forensic treatment.

In conclusion, more empirical studies and validated theoretical models are needed concerning the treatment of psychopathy to better inform and guide clinical practice and policy decisions, especially in Dutch forensic psychiatry. 
SUMMARY

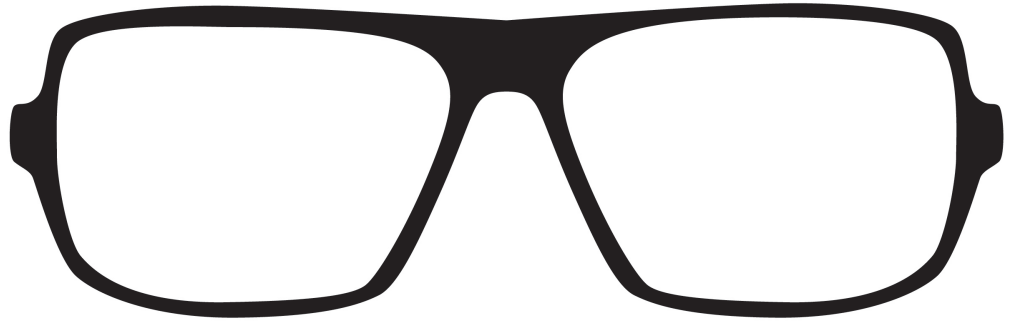

10 


\section{SUMMARY}

\section{Background}

Psychopathy is commonly defined as a personality disorder characterized by a lack of empathy and remorse, a manipulative interpersonal style, poor behavioral controls and frequent antisocial behavior (Hare, 2003). From its first conceptualization in modern psychiatry, psychopathy has been surrounded with therapeutic pessimism (Cleckley, 1941; D'Silva, Duggan, \& McCarthy, 2004; Salekin, Worley, \& Grimes, 2010). Many experts believe that these characteristics are difficult, if not impossible, to ameliorate (Harris \& Rice, 2006), and the findings of some studies suggested that treatment makes psychopaths recidivate more (Hare, Clark, Grann, \& Thornton, 2000; Rice, Harris, \& Cormier, 1992; Seto \& Barbaree, 1999).

Psychopathy, as measured with the Psychopathy Checklist-Revised (PCL-R; Hare, 1991, 2003) is considered to be among the most important constructs in forensic psychiatry. Psychopathy is associated with higher recidivism rates after imprisonment or forensic treatment (Hemphill, Hare, \& Wong, 1998; Leistico, Salekin, DeCoster, \& Rogers, 2008) and is highly prevalent in Dutch forensic psychiatry, affecting 24-35 percent of the population (Hildebrand, Hesper, Spreen, \& Nijman, 2005; Hildebrand \& de Ruiter, 2004). Thus, psychopathy poses a considerable challenge for forensic treatment settings in fulfilling their aim which is protecting society from the risk of re-offending by mentally disordered offenders. This challenge has given rise to conceptions of withholding treatment from psychopathic patients or admitting them indefinitely, within the Dutch forensic psychiatric regime, to so-called long-stay facilities. At the same time, psychopathy represents a personality disorder which, to this date, has not been studied with rigorous research designs, such as randomized clinical trials, examining treatment responsiveness. In addition, the results of previous studies suggesting that treatment makes psychopaths worse, are generally accepted as methodologically inadequate (D'Silva et al., 2004; Salekin et al., 2010; Skeem, Polaschek, Patrick, \& Lilienfeld, 2011). Therefore, several authors have argued the great need for further understanding, assessment and treatment of psychopathy, by conducting pilot studies, case studies, and treatment effectiveness studies of a variety of approaches to the assessment and treatment of this disorder (e.g., Hart, 2011).

During the last decade, an innovative approach to the treatment of personality disorders called Schema Therapy (ST; Rafaeli, Bernstein, \& Young, 2011; Young, Klosko, \& Weishaar, 2003), has shown effectiveness in randomized and open clinical trials of patients with borderline personality disorder (Farrell, Shaw, \& Webber, 2009; Giesen-Bloo et al., 2006; Nadort et al., 2009). These findings support the changing perspective on the treatment of borderline personality disorder which had long been considered untreatable (Arntz \& Bernstein, 2006), and lend support to examining ST as a framework to further our understanding of the assessment and treatment of personality disorders in forensic settings, including psychopathy (Bernstein, Arntz, \& de Vos, 2007). ST was specifically developed for patients with severe personality disorders who failed to respond to, or relapsed after, traditional cognitive therapy (Young et al., 2003), and has recently been adapted for use with forensic patients with personality disorders (Bernstein et 
al., 2007; 2012). One of the main targets of ST is the change of chronic emotional and cognitive maladaptive patterns, called early maladaptive schemas (EMS), that originate in adverse childhood experiences and early temperament, and that affect emotional processing, influence interpersonal interactions and guide behavior (Young et al., 2003). Young and colleagues (2003) hypothesized that EMS lie at the core of personality disorders, and externalizing behaviors, such as anger and hostility towards others, are primarily maladaptive coping responses to EMS.

Little is known about the relationship between the cognitive sequelae of adverse childhood experiences and (the development of) psychopathy. There is some empirical evidence that lends support to examining the presence of EMS in psychopathy. For example, adverse childhood experiences, are presumed to give rise to the development of EMS, are associated with the development of psychopathic traits in adulthood (e.g., Weiler \& Widom, 1996), and several studies have shown that childhood trauma is associated with higher PCL-R scores in offender samples (e.g., Marshall \& Cooke, 1999).

The studies reported in this thesis were designed to examine the presence of EMS in offenders with psychopathic traits, including sexual offenders, to examine treatment responsiveness of offenders with psychopathic traits, and to examine if ST could be useful in the treatment of psychopathic traits.

\section{Summary of the chapters}

Chapter 1 presents an introduction to the subsequent chapters. First, the conceptualization of psychopathy as measured with the PCL-R is presented. Second, the research evidence that contributed to the therapeutic pessimism regarding the treatability of psychopathic personality disorder is reviewed. Third, Schema Therapy is presented as a promising approach to the treatment of psychopathy. It is discussed how EMS may be relevant in understanding offenders with psychopathic traits, including sexual offenders. Fourth, giving the well-known tendency for impression management in psychopaths, the need for an observational instrument that measures change on risk-related behaviors during forensic treatment is discussed. Finally, a brief description is provided of the setting in which the research studies were conducted.

The present dissertation contains five empirical studies, organized in two parts. The first part is focused on assessment and comprises chapters 2, 3 and 4 . The second part is focused on treatment and comprises chapters 5 and 6 .

\section{Part I: Assessment}

The aim of the prospective study described in Chapter 2 was to examine the value of the Behavioural Status Index (BEST-Index) as an instrument for measuring change on risk-related behaviors during forensic psychiatric treatment. The BESTIndex is a structured nurse-rated assessment tool for the assessment of aggressive behavior, degree of insight and social skills during inpatient treatment. The reliability and validity of the BEST-Index was examined in a sample of 291 male mentally disordered offenders and rated by 182 forensic nurses. Findings showed that the BEST-Index can be used reliably and it was significantly associated with risk of future violence as measured with the Historical Clinical Risk Management-20 (HCR-20; Webster, Douglas, Eaves, \& Hart, 1997). Furthermore, the study revealed 
a different and clearer factor structure for the BEST-Index in our sample, compared to the original one. The revised BEST-Index predicted inpatient aggressive behavior comparable to, if not better than, the HCR-20. The findings of the study support the use of the BEST-Index for measuring change in risk-related behaviors during forensic psychiatric treatment. In addition self-report measures, the use of the BEST-Index may circumvent common problems associated with the assessment of forensic psychiatric patients, such as socially desirable responding and impression management.

In Chapter 3, we investigated the hypotheses that EMS are related to psychopathic traits and institutional violence in adult male personality disordered offenders. Young and colleagues (2003) offered no specific hypotheses about EMS that may be prevalent in psychopathy. However, following Beck and colleagues' (1990) theory on maladaptive cognitions in antisocial personality disorder (APD), we hypothesized that the EMS Mistrust/Abuse, Emotional Inhibition, Entitlement, and Insufficient Self-Control would predict PCL-R Impulsive Lifestyle and Antisocial Facets, and institutional violence. A sample of 124 personality disordered offenders was assessed with the PCL-R and the Young Schema Questionnaire. Information about institutional violence was gathered over a follow-up period of two years using a modified version of the Overt Aggression Scale for use in forensic practice (Nicholls, Brink, Desmarais, Webster, \& Martin, 2006). The results showed that the PCL-R Lifestyle and Antisocial Facets were significantly related to the EMS Mistrust/Abuse and Insufficient Self-Control, consistent with our hypotheses, and were significantly, but negatively, related to the EMS Subjugation. Also, as hypothesized, EMS showed no associations with the PCL-R Affective and Interpersonal facets. Contrary to our expectation, EMS did not predict institutional violence.

These findings suggested that EMS related to mistrust, inadequate selfcontrol, low frustration tolerance, and autonomy and dominance (the inverse relationship with Subjugation), play a role in the impulsive lifestyle and antisocial behavior facets of psychopathy. Our findings are consistent with previous research in personality disordered individuals that showed that the severity of APD symptoms is associated with EMS (Ball \& Cecero, 2001). Although - to our knowledge - this study was one of the first to examine EMS in psychopaths, and caution should be exercised in generalizing these results, our findings suggest that treatments which target maladaptive schemas may enable some psychopaths to develop more balanced and accurate appraisals of other persons' intentions, and to better cope with situations that would otherwise have triggered schema-related reactive aggression.

In Chapter 4, we explored the presence of EMS in child sexual offenders compared to sexual offenders against adults and nonsexually violent offenders. In light of previous research that suggested that child sexual offenders were more likely to have a history of physical and sexual abuse than other offenders (e.g., Salter et al., 2003), and the literature on cognitive distortions in child sexual offenders (e.g., Ó Ciardha \& Gannon, 2011), we hypothesized that child sexual offenders were more likely to endorse the EMS Social Isolation, Emotional Deprivation and Vulnerability to Harm compared to sexual offenders against adults. Also, we hypothesized that child sexual offenders were more likely to endorse EMS 
related to Abandonment and Mistrust/Abuse compared to nonsexual violent offenders. In a sample of 23 child sexual offenders, 19 sexual offenders against adults and 24 nonsexual violent offenders, we assessed EMS with the Young Schema Questionnaire, and psychopathy with the PCL-R. The findings showed that, after controlling for level of psychopathy, EMS related to Abandonment, Social Isolation, Defectiveness/Shame, Subjugation and Self-Sacrifice were more prevalent in child sexual offenders compared to nonsexual violent offenders. Compared to sexual offenders against adults, child sexual offenders showed a trend to have higher scores on EMS related to Social Isolation. Although our hypotheses were not fully supported, our findings revealed a strikingly consistent pattern of EMS among the three offender groups. The child sexual offenders consistently demonstrated the highest scores on the EMS domains, the nonsexual violent offenders consistently demonstrated the lowest scores, but the EMS domain scores of the child sexual offenders and sexual offenders against adults did not differ significantly from each other. These findings suggest that EMS can inform the assessment of sexual offenders which is currently focused largely on cognitive distortions and post-hoc rationalizations of sexual offending (Maruna \& Mann, 2006). Young's theory on EMS may offer a broader understanding of developmental and cognitive processes underlying the distortions and rationalizations present in sexual offenders.

\section{Part II: Treatment}

The prospective study in Chapter 5 examined change during forensic treatment in a sample male personality disordered offenders, differentiated according to their total score on Hare's PCL-R, into psychopathic and nonpsychopathic cases. In light of the research on treatment outcome in psychopaths, we hypothesized that psychopaths would show the same treatment response as nonpsychopaths. In addition, we predicted on an individual level, that similar percentages of psychopaths and nonpsychopaths would show reliable improvement, no improvement, or deterioration. Furthermore, we hypothesized that within the group of psychopaths, treatment responsiveness varies and that individual variability within psychopaths is masked in group level analyses.

The offenders were assessed six-monthly over a two-year course by means of the BEST-Index, on adaptive social behavior, communication skills, insight, attribution of responsibility, self-regulation strategies and aggressive behavior. Both the group- and individual level analyses showed few significant differences between psychopaths and nonpsychopaths in terms of degree of change. Psychopaths, as well as nonpsychopaths, showed change on BEST-Index subscales that measure adaptive social behavior, communication skills, insight and attribution of responsibility. The only significant difference was found in higher acts of physical violence displayed by psychopaths compared to nonpsychopaths. More specifically, nearly a quarter (22\%) of the psychopaths showed reliable deterioration, whereas nonpsychopaths showed no reliable deterioration in acts of physical violence during treatment. Also, the findings confirmed our hypothesis that within the group of psychopaths treatment responsiveness varies greatly. Specifically, approximately one third of the psychopaths showed reliable improvement on adaptive social behavior, communication skills, insight, attribution of responsibility, and self- 
regulation strategies. These findings demonstrate that, contrary to clinical lore, treatment did not make all or a majority of the psychopaths worse. Our findings, as well as those of some previous studies (see D'Silva et al., 2004; Salekin et al., 2010), suggest that treatment should not be routinely withheld from forensic patients based on their high PCL-R scores. Although psychopaths do show higher rates of recidivism than other forensic patients, this does not imply they are untreatable.

Chapter 6 presents the results of a single case study of a male forensic patient with psychopathic features. To our knowledge, this is the first case study of a psychopathic patient treated with Schema Therapy (ST) in a forensic psychiatric hospital. We describe the 4-year ST treatment and 3-year follow-up of a 25-year old patient with a pre-treatment PCL-R total score of 28.4. The ST treatment process is described narratively with key ST interventions and mechanisms of change highlighted. Change during treatment was assessed using independent assessments of psychopathic traits (PCL-R), cognitive schemas (Young Schema Questionnaire), and risk-related behaviors (BEST-Index; HCR-20) over the 4-year treatment period. Reliable change analyses showed significant improvements in psychopathic traits, cognitive schemas, and risk-related outcomes. As treatment of psychopathy should be evaluated by an outcome measure of psychopathic traits, the PCL-R was administered pre- and post-treatment. By the end of the ST treatment, the patient's scores on PCL-R items for prominent psychopathic features such as lack of empathy, impulsivity, and failure to take responsibility, were reduced (post-treatment $P C L-R$ total score $=14$ ). At 3 years post-treatment, the patient was living independently outside the forensic institution without judicial supervision and he had not reoffended according to official justice records. While many questions about the nature of change in psychopathic patients remain, this case study detailed the first apparently successful ST treatment of a psychopathic patient. This study challenges the widely held view that patients with psychopathic features cannot be treated, or that treatment makes them worse (Rice et al., 1992; Seto \& Barbaree, 1999). An ongoing randomized clinical trial (Bernstein et al., 2012) will show whether ST proves to be an empirically-supported treatment for this challenging patient population.

Finally, Chapter 7 summarizes the most important findings of the studies in this thesis. The strengths and limitations of the empirical studies are discussed. Next, the clinical and policy implications for forensic clinical practice are discussed. For example, it is suggested that utilizing a more general theory of psychopathology, in this case Young's Schema Therapy framework (Young et al., 2003; Bernstein et al., 2007), may further our understanding of the assessment and treatment of psychopathy. By explaining a patients' pathology from a developmental psychopathology perspective, looking beyond the pejorative label of psychopathy, this approach may enhance the forensic patient's motivation and responsivity for forensic treatment. In addition, it is suggested that policy makers and mental health professionals should avoid to equate psychopathy with untreatability as reflected in existing criteria for decisions regarding suspension of leave privileges and admitting patients to long-stay facilities. The chapter is concluded with suggestions for future research regarding the theoretical assumptions between EMS and aggression in offenders and regarding mechanisms of change in Schema Therapy. 


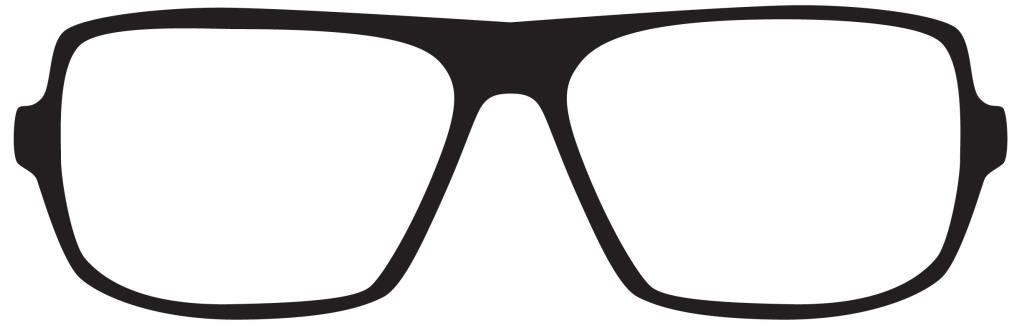




\section{SAMENVATTING}

\section{Achtergrond}

Psychopathie wordt gedefinieerd als een persoonlijkheidsstoornis, welke wordt gekenmerkt door een gebrek aan empathie en berouw, een manipulatieve interpersoonlijke stijl, gebrekkige woedebeheersing en frequent antisociaal gedrag (Hare, 2003). Het construct psychopathie is van meet af aan in verband gebracht met therapeutisch pessimisme (Cleckley, 1941; D'Silva, Duggan, \& McCarthy, 2004; Salekin, Worley, \& Grimes, 2010). Veel deskundigen zijn van mening dat behandeling van deze persoonlijkheidsstoornis moeilijk, zo niet onmogelijk, is (Harris \& Rice, 2006) en de bevindingen van enkele studies lijken te suggereren dat psychopaten juist meer gaan recidiveren met behandeling dan zonder (Hare, Clark, Grann, \& Thornton, 2000; Rice, Harris, \& Cormier, 1992; Seto \& Barbaree, 1999).

Psychopathie, zoals vastgesteld met de Psychopathie Checklist-Revised (PCLR; Hare, 1991, 2003) wordt gezien als een van de belangrijkste begrippen in de forensische psychiatrie. Psychopathie hangt samen met een hoge recidive na detentie of forensische behandeling (Hemphill, Hare, \& Wong, 1998; Leistico, Salekin, DeCoster, \& Rogers, 2008). De forensische psychiatrie heeft als taak het beschermen van de maatschappij tegen het risico van recidive, naast het bieden van adequate zorg aan de patient-delinquent. De prevalentie van psychopathie in Nederlandse forensische psychiatrische populaties ligt tussen de 24 en 35 procent (Hildebrand, Hesper, Spreen, \& Nijman, 2005; Hildebrand \& de Ruiter, 2004). Bovenstaande bevindingen hebben geleid tot de visie om behandeling aan psychopaten te weigeren of om hen voor onbepaalde tijd op te nemen binnen zogenaamde long-stay afdelingen in de Nederlandse forensisch psychiatrische sector. Tegelijkertijd is psychopathie een persoonlijkheidsstoornis waarbij, tot op heden, geen gedegen wetenschappelijk onderzoek, zoals gerandomiseerde effectstudies, is verricht naar de respons op behandeling. Daarnaast worden de eerdere studies, waarvan de bevindingen suggereerden dat behandeling psychopaten gevaarlijker maakt, in het algemeen beschouwd als methodologisch ontoereikend (D'Silva et al., 2004; Salekin et al., 2010; Skeem, Polaschek, Patrick, \& Lilienfeld, 2011). Daarom is het essentieel om de kennis over de behandelbaarheid van psychopathie zoveel mogelijk te verbeteren door het uitvoeren van pilotstudies, case-studies en studies naar behandeleffecten van verschillende benaderingen voor behandeling van deze persoonlijkheidsstoornis (Hart, 2011).

In het afgelopen decennium is een innovatieve benadering voor de behandeling van persoonlijkheidsstoornissen, genaamd Schema Therapie (ST; Rafaeli, Bernstein, \& Young, 2011; Young, Klosko, \& Weishaar, 2003), effectivitief gebleken in enkele gerandomiseerde effectstudies bij patiënten met een borderline persoonlijkheidsstoornis (Farrell, Shaw, \& Webber, 2009; Giesen-Bloo et al., 2006; Nadort et al., 2009). Deze bevindingen ondersteunen de veranderende kijk op de behandeling van de borderline persoonlijkheidsstoornis, een stoornis die lange tijd werd beschouwd als onbehandelbaar (Arntz \& Bernstein, 2006). Ook ondersteunen zij het initiatief om ST verder te onderzoeken als theoretisch kader voor de behandeling van persoonlijkheidsstoornissen, inclusief psychopathie, in forensische 
instellingen (Bernstein, Arntz, \& de Vos, 2007). ST is specifiek ontwikkeld voor patiënten met ernstige persoonlijkheidsstoornissen die niet reageerden op, of terugvielen in klachten na traditionele cognitieve gedragstherapie (Young et al., 2003) en is onlangs aangepast voor het gebruik bij forensische patiënten met persoonlijkheidsstoornissen (Bernstein et al., 2007; 2012). Een van de belangrijkste doelen van ST is het veranderen van persisterende emotionele en cognitieve maladaptieve patronen, de zogenaamde vroeg ontwikkelde onaangepaste schema's (VOS). Volgens de theorie hebben VOS, die hun oorsprong vinden in traumatische ervaringen in de kindertijd en in aangeboren karaktereigenschappen, een grote invloed op de verwerking van emoties, de interacties met anderen en zijn ze sturend in het gedrag van een individu (Young et al., 2003). Young en collega's (2003) stellen dat VOS de kern vormen van een persoonlijkheidsstoornis en dat externaliserende gedragingen (bijv. agressie tegen anderen) emotionele en cognitieve reacties zijn ten gevolge van de VOS.

$\mathrm{Er}$ is weinig bekend over de relatie tussen de cognitieve gevolgen van traumatische ervaringen in de kindertijd en (de ontwikkeling van) psychopathie. Wel zijn er aanwijzingen die onderzoek naar de aanwezigheid van VOS bij psychopaten verder ondersteunen. Zo worden traumatische ervaringen in de jeugd - waarvan wordt verondersteld dat ze leiden tot de ontwikkeling van VOS - in verband gebracht met de ontwikkeling van psychopathische trekken in de volwassenheid (bijv., Weiler $\&$ Widom, 1996) en blijken vroege traumatische ervaringen samen te hangen met hogere PCL-R scores in populaties delinquenten (bijv., Marshall \& Cooke, 1999).

De studies in dit proefschrift zijn opgezet om: de aanwezigheid van VOS te onderzoeken bij forensische patienten met psychopathische trekken, zowel bij daders van geweld- als seksuele delicten; de behandelrespons van patienten met psychopathische trekken te onderzoeken en; om te onderzoeken of Schema Therapie een geschikte vorm van behandeling is voor psychopathische kenmerken bij mannelijke forensisch psychiatrische patiënten.

\section{Samenvatting van de hoofdstukken}

Hoofdstuk 1 biedt een inleiding op de daaropvolgende hoofdstukken. Ten eerste wordt de conceptualisatie van psychopathie, zoals gemeten met de PCL-R, gepresenteerd. Ten tweede wordt het eerdere onderzoek dat bijdroeg aan het therapeutische pessimisme rondom de behandeling van psychopathie besproken. Ten derde wordt Schema Therapie gepresenteerd als een mogelijke benadering voor de behandeling van psychopathie. Er wordt uitgelegd waarom VOS relevant zou kunnen zijn voor patienten met psychopathische kenmerken, met inbegrip van daders van seksuele delicten. Ten vierde wordt, omdat psychopaten bekend zijn om hun neiging tot het geven van sociaal wenselijke antwoorden, de noodzaak uitgesproken voor een observatie-instrument dat veranderingen op risico-gerelateerd gedrag tijdens de behandeling kan vaststellen. Ten slotte wordt een korte beschrijving gegeven van de setting waarin de onderzoeken uit deze dissertatie zijn uitgevoerd.

Dit proefschrift bevat vijf empirische studies, georganiseerd in twee delen. Het eerste deel is gericht op diagnostiek en bestaat uit de hoofdstukken 2, 3 en 4 . Het tweede deel is gericht op behandeling en bestaat uit de hoofdstukken 5 en 6 . 


\section{Deel I: Diagnostiek}

Het doel van de prospectieve studie die beschreven wordt in Hoofdstuk 2 was om de waarde van de Behavioural Status Index (BEST-Index) te onderzoeken als instrument voor het meten van veranderingen in belangrijke risico-indicatoren gedurende de forensische psychiatrische behandeling. De BEST-Index is een gestructureerd observatie instrument voor de beoordeling van agressief gedrag, en de mate van inzicht en sociale vaardigheden. De betrouwbaarheid en validiteit van de BEST-index werd onderzocht in een steekproef van 291 mannelijke forensisch psychiatrische patienten en werd gescoord door 182 sociotherapeuten. De resultaten toonden aan dat de BEST-index op betrouwbare wijze gescoord kon worden en dat de BEST-Index significant samenhing met het risico op toekomstig geweld zoals gemeten met de Historical Clinical Risk Management-20 (HCR-20; Webster, Douglas, Eaves, \& Hart, 1997). Bovendien kwam in de onderzochte steekproef een andere en duidelijkere factorstructuur naar voren in vergelijking tot de oorspronkelijke factorstructuur (Reed, Woods, \& Robinson, 2000). De factoren van de gereviseerde versie van de BEST-Index voorspelde agressief gedrag in de instelling vergelijkbaar met, of beter dan, de HCR-20. De bevindingen van deze studie ondersteunen het gebruik van de BEST-index voor het meten van veranderingen in risico-gerelateerd gedrag tijdens forensische psychiatrische behandeling. Naast zelfrapportage methoden kan aanvullend gebruik van de BESTindex veelvoorkomende problemen, zoals sociale wenselijkheid, bij de diagnostiek van forensisch psychiatrische patiënten omzeilen.

In Hoofdstuk 3 werd de hypothese onderzocht dat VOS gerelateerd zouden zijn aan psychopathische kenmerken en aan institutioneel geweld in een groep volwassen mannelijke persoonlijkheidsgestoorde forensische patienten. Young en collega's (2003) formuleerden geen specifieke hypothesen over VOS die mogelijk aanwezig zijn bij psychopathie. Echter, in navolging van de theorie van Beck en collega's (1990) over maladaptieve schema's bij individuen met een antisociale persoonlijkheidsstoornis (APS), was onze hypothese dat de VOS Wantrouwen / misbruik, Emotionele inhibitie, Gerechtigheid en Onvoldoende Zelfcontrole geassocieerd zouden zijn met de PCL-R facetten Impulsieve levensstijl en Antisociaal gedrag en met institutioneel geweld. In een steekproef van 124 persoonlijkheidsgestoorde patienten werd de PCL-R gecodeerd en de Young Schema Vragenlijst afgenomen. Informatie over agressie in de kliniek werd verzameld over een follow-up periode van twee jaar met behulp van een, voor gebruik in de forensische praktijk, aangepaste versie van de Overt Aggression Scale (Nicholls, Brink, Desmarais, Webster, \& Martin, 2006). De resultaten lieten zien dat de PCL-R facetten Impulsieve Levensstijl en Antisociaal gedrag in overeenstemming met onze hypothesen significant gerelateerd waren aan de VOS Wantrouwen / misbruik en Onvoldoende Zelfcontrole, en significant negatief gerelateerd waren aan de VOS Onderwerping. Eveneens volgens onze verwachtingen, vertoonde de VOS geen relatie met de Affectieve en Interpersoonlijke facetten van de PCL-R. In tegenstelling tot onze hypothese bleken de VOS geen relatie te vertonen met institutioneel geweld.

Deze bevindingen suggereren dat VOS met betrekking tot wantrouwen, onvoldoende zelfcontrole, lage frustratietolerantie en autonomie / dominantie (de negatieve relatie met Onderwerping) een rol spelen bij de impulsieve levensstijl en 
de antisociale gedragskenmerken van psychopathie. Onze bevindingen zijn consistent met eerder onderzoek bij persoonlijkheidsgestoorde individuen waaruit bleek dat de ernst van de symptomen van de Antisociale Persoonlijkheidsstoornis samenhing met de aanwezigheid van VOS (Ball \& Cecero, 2001). Hoewel onze studie - voor zover wij weten - een van de eerste studies is die de aanwezigheid van VOS onderzocht in relatie tot psychopatie en voorzichtigheid derhalve geboden is bij het generaliseren van deze resultaten, suggereren onze bevindingen dat behandeling die zich richt op maladaptieve schema's patienten met psychopathie mogelijk kan helpen om meer evenwichtige beoordelingen van de intenties van anderen te maken en ze kan helpen om beter om te gaan met situaties die veelal leiden tot het uiten van schema-gerelateerde reactieve agressie.

In Hoofdstuk 4 hebben we de aanwezigheid van VOS bij daders van seksuele delicten tegen kinderen vergeleken met daders van seksueel geweld tegen volwassenen en daders van niet-seksueel geweld. Eerder onderzoek suggereert dat daders van seksueel geweld tegen kinderen vaker een voorgeschiedenis van lichamelijke en seksuele mishandeling rapporteren dan andere daders (bijv., Salter et al., 2003). Aan de hand van deze bevindingen en de literatuur over cognitieve vervormingen bij daders van seksueel geweld tegen kinderen (bijv., Ó Ciardha \& Gannon, 2011), werden twee hypothesen geformuleerd: de eerste hypothese was dat daders van seksueel geweld tegen kinderen eerder geneigd zijn de VOS Sociaal isolement, Emotionele deprivatie en Kwetsbaarheid voor gevaar te rapporteren in vergelijking met daders van seksueel geweld tegen volwassenen. De tweede hypothese was dat daders van seksueel geweld tegen kinderen eerder geneigd zijn de VOS Verlating/instabiliteit en Wantrouwen/misbruik te rapporteren in vergelijking met daders van niet-seksueel geweld. In een steekproef van 23 daders van seksueel geweld tegen kinderen, 19 daders van seksueel geweld tegen volwassenen en 24 daders van niet-seksuele geweldsdelicten werden de VOS gemeten met behulp van de Young Schema vragenlijst en psychopathie met de PCLR. De resultaten toonden aan, nadat was gecontroleerd voor het effect van psychopathie, dat de VOS met betrekking tot Verlating/instabiliteit, Sociaal isolement, Tekortschieten/schaamte, Onderwerping en Zelfopoffering vaker gerapporteerd werden door de daders van seksueel geweld tegen kinderen in vergelijking met de daders van niet-seksueel geweld. In vergelijking met daders van seksueel geweld tegen volwassenen vertoonden daders van seksueel geweld tegen kinderen een trend naar hogere scores op de VOS Sociaal Isolement. Hoewel onze hypothesen niet volledig werden ondersteund, was in onze resultaten een opvallend consistent patroon in de VOS tussen de drie dadergroepen te zien. De daders van seksueel geweld tegen kinderen hadden consistent de hoogste scores op VOS domeinen, de daders van niet-seksueel geweld rapporteerden consistent de laagste scores. De scores op de VOS domeinen van de daders van seksueel geweld tegen kinderen en de daders van seksueel geweld tegen volwassenen bleken niet significant van elkaar te verschillen. Deze bevindingen suggereren dat de VOS een meerwaarde kunnen hebben in de diagnostiek en behandeling van seksuele geweldplegers die momenteel veelal gericht is op cognitieve vervormingen die direct samenhangen met het delictgedrag en met post-hoc rationalisaties van het delictgedrag (bijv., "de drang was sterker dan mijzelf"; Maruna \& Mann, 2006). Youngs theorie over VOS kan wellicht leiden tot beter begrip van (de ontwikkeling 
van de) processen die ten grondslag liggen aan de cognitieve vervormingen en posthoc rationalisaties bij seksuele geweldplegers.

\section{Deel II: Behandeling}

In de prospectieve studie uit Hoofdstuk 5 werd de verandering tijdens de forensische behandeling onderzocht bij patienten met persoonlijkheidsstoornissen die, op basis van hun totaalscore op Hare's PCL-R, werden ingedeeld in psychopathische en niet-psychopathische patienten. Op basis van eerder onderzoek naar behandeluitkomsten bij psychopathie was de hypothese dat psychopaten een vergelijkbare behandelrespons zouden laten zien als niet-psychopaten. Ook was onze voorspelling dat op individueel niveau, vergelijkbare percentages psychopaten en niet-psychopaten een betrouwbare verbetering, geen verbetering of een betrouwbare verslechtering zouden laten zien. Daarnaast toetsten we de hypothese dat binnen de groep psychopaten, individuele verschillen zouden bestaan in behandelrespons die niet zichtbaar zijn in analyses op groepsniveau.

De patienten werden halfjaarlijks beoordeeld over een periode van twee jaar, aan de hand van de BEST-index (adaptief sociaal gedrag, communicatieve vaardigheden, inzicht, het nemen van verantwoordelijkheid, zelfregulerende strategieën en agressief gedrag). Zowel in de groepsanalyses als in de analyses op individueel niveau waren er geen significante verschillen in de voortgang tijdens de behandeling tussen de psychopathische en de niet-psychopathische patiënten op adaptief sociaal gedrag, communicatieve vaardigheden, inzicht, het nemen van verantwoordelijkheid en zelfreguleringsstrategieën. Echter, een subgroep van de psychopaten (22\%) verslechterde tijdens de behandeling met betrekking tot fysieke agressie terwijl geen van de niet-psychopaten hierop verslechterde. Ook bevestigden de resultaten onze hypothese dat binnen de groep psychopaten de behandelrespons varieert. Ongeveer een derde van de psychopaten liet een betrouwbare verbetering zien op adaptief sociaal gedrag, communicatieve vaardigheden, inzicht, het nemen van verantwoordelijkheid en zelfreguleringsstrategieën. De resultaten van deze studie toonden aan dat psychopaten als groep niet gevaarlijker worden door behandeling. Onze bevindingen, evenals die van een aantal eerdere studies (zie D'Silva et al., 2004; Salekin et al., 2010), suggereren dat behandeling niet geweigerd behoort te worden aan forensische patiënten op basis van hun hoge PCL-R score. Hoewel psychopaten hogere recidive cijfers laten zien, betekent dit niet dat ze onbehandelbaar zijn.

Hoofdstuk 6 beschrijft de resultaten van een gevalsstudie bij een mannelijke forensisch psychiatrische patiënt met psychopathische kenmerken. Voor zover wij weten, is dit de eerste gevalsbeschrijving van een psychopathische patiënt behandeld met Schema Therapie (ST) in een forensisch psychiatrische instelling. In deze studie werd de ST behandeling van 4 jaar beschreven, met een 3-jaar followup, bij een 25-jarige patiënt met een PCL-R totaalscore van 28.4. Ook werden de belangrijkste ST interventies en veranderingsmechanismen tijdens de therapie gedetailleerd beschreven. De veranderingen tijdens de behandeling werden vastgesteld met behulp van een onafhankelijke beoordeling van psychopathie (PCL$\mathrm{R})$, cognitieve schema's (Young Schema vragenlijst) en risico-gerelateerd gedrag (HCR-20; BEST-Index) gedurende de 4-jarige behandelperiode. Reliable Change analyses toonden significante verbeteringen aan in de psychopathische kenmerken, 
cognitieve schema's en de risico-gerelateerde gedragingen. Omdat de behandeling van psychopathie dient te worden beoordeeld aan de hand van verandering in de psychopathische kenmerken zelf, werd de PCL-R voor en na de behandeling afgenomen. Na beëindiging van de ST behandeling waren de scores van de patiënt op psychopathische kenmerken, zoals een gebrek aan empathie, impulsiviteit en het niet nemen van verantwoordelijkheid voor het eigen gedrag, sterk afgenomen (PCL-R totaalscore van 14 aan het einde van de behandeling). Drie jaar na beëindiging van de behandeling woonde de patiënt zelfstandig buiten de forensische instelling zonder vorm van toezicht en was hij niet gerecidiveerd volgens officiële justitiële bronnen. Ook deze studie spreekt de wijdverbreide mening tegen dat patiënten met psychopathische kenmerken niet reageren op behandeling of dat behandeling hen gevaarlijker maakt (Rice et al., 1992;. Seto \& Barbaree, 1999). Een lopende gerandomiseerde effectstudie (Bernstein et al., 2012) zal nog moeten uitwijzen of Schema Therapie een empirisch ondersteunde behandeling is voor deze uitdagende patiëntenpopulatie.

Tenslotte worden in Hoofdstuk 7 de meest belangrijke bevindingen uit dit proefschrift samengevat. De sterke punten en beperkingen van de empirische studies worden besproken, evenals suggesties voor toekomstig onderzoek en de klinische- en beleidsimplicaties voor de forensische praktijk. Zo wordt geadviseerd dat het gebruik van een algemene theorie van psychopathologie, zoals Young's schema theorie (Young et al., 2003; Bernstein et al., 2007), kan bijdragen aan een betere diagnostiek en behandeling van psychopathie. Ook wordt aangeraden de motivatie en responsiviteit van patiënten voor forensische behandeling te bevorderen door de stoornis psychopathie mede in een ontwikkelingspsychopathologisch perspectief te plaatsen. Daarnaast wordt beleidsmakers en hulpverleners geadviseerd om psychopathie niet gelijk te stellen aan onbehandelbaarheid, met gevolgen voor de huidige criteria voor verlofverlening aan forensische patiënten en voor de langdurige opname in long-stay faciliteiten. Het hoofdstuk wordt afgesloten met suggesties voor toekomstig onderzoek naar de theoretische aannamen over de relatie tussen VOS en agressie, en met betrekking tot onderzoek gericht op de veranderingsmechanismen in de Schema Therapie. 
REFERENCES

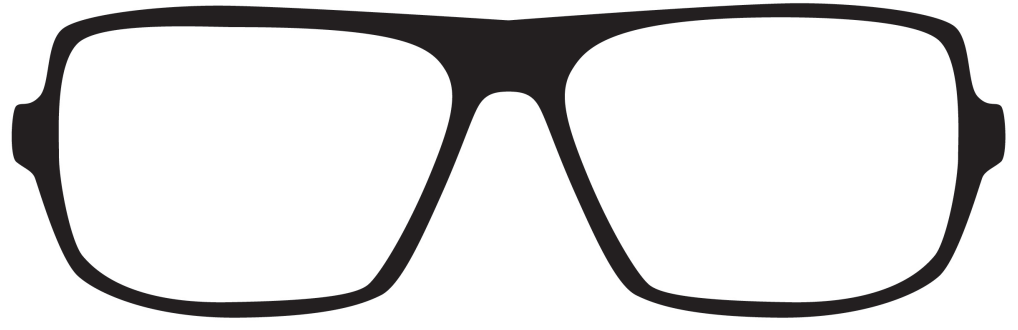


Adviescollege Verloftoetsing TBS [AVT] (2012). Jaarverslag 2011. Utrecht: AVT.

American Psychiatric Association (2000). Diagnostic and statistical manual of mental disorders (Revised 4th ed.). Washington, DC: Author.

Arbuckle, J. L. (2006). Amos (Version 7.0) [Computer Software]. Chicago: SPSS.

Arntz, A., \& Bernstein, D. P. (2006). Can personality disorders be changed? Netherlands Journal of Psychology, 62, 8-18.

Atkins, D. C., Bedics, J., McGlinchey, J. B., \& Beauchaine, T. P. (2005). Assessing clinical significance: Does it matter which method we use? Journal of Consulting and Clinical Psychology, 73, 982-989.

Ball, S. A. (2007). Comparing individual therapies for personality disordered opioid dependent patients. Journal of Personality Disorders, 21, 305-321.

Ball, S. A., \& Cecero, J. J. (2001). Addicted patients with personality disorders: Traits, schemas, and presenting problems. Journal of Personality Disorders, 15, 72-83.

Barbaree, H. E. (2005). Psychopathy, Treatment Behavior, and Recidivism: An Extended Follow-Up of Seto and Barbaree. Journal of Interpersonal Violence, 20, 1115-1131.

Barker, E., \& Buck, M. F. (1977). LSD in a coercive milieu therapy program. Canadian Psychiatric Association Journal, 22, 311-314.

Barker, E., \& McLaughlin, A. J. (1977). The total encounter capsule. Canadian Psychiatric Association Journal, 22, 355-360.

Barker, E., Mason, M., \& Wilson, J. (1969). Defence-disrupting therapy. Canadian Psychiatric Association Journal, 14, 355-359.

Beck, A. T., Freeman, A., \& Associates. (1990). Cognitive therapy of personality disorders. New York: Guilford Press.

Bernstein, D. P., Arntz, A., \& de Vos, M. E. (2007). Schema-Focused Therapy in forensic settings. International Journal of Forensic Mental Health, 6, 169-183.

Bernstein, D. P., \& Fink, L. (1998). Childhood trauma questionnaire: A retrospective self-report manual. San Antonio, TX: The Psychological Corporation.

Bernstein, D. P., Nijman, H. L. I., Karos, K., Keulen-de Vos, M. E., de Vogel, V., \& Lucker, T. P. (2012). Schema therapy for forensic patients with personality disorders: Design and preliminary findings of a multicenter randomized clinical trial in the Netherlands. International Journal of Forensic Mental Health, 11, 312-324.

Blair, R. J. R. (2003). Neurobiological basis of psychopathy. British Journal of Psychiatry, 182, 5-7. 
Blair, R. J. R., \& Mitchell, D. V. G. (2009). Psychopathy, attention and emotion. Psychological Medicine, 39, 543-555.

Boccaccini, M. T., Murrie, D. C., Clark, J., \& Cornell, D. (2008). Describing, diagnosing and naming psychopathy: How do youth psychopathy labels influence jurors? Behavioral Sciences and the Law, 26, 487-510.

Boer, D. P., Hart, S. D., Kropp, P. R., \& Webster, C. D. (1997). Manual for the Sexual Violence Risk - 20: Professional guidelines for assessing risk of sexual violence. Vancouver: The Mental Health, Law, \& Policy Institute.

Bonta, J., Law, M., \& Hanson, R. K. (1998). The prediction of criminal and violent recidivism among mentally disordered offenders: A meta-analysis. Psychological Bulletin, 123, 123-142.

Bosmans, G., Braet, C., \& van Vlierberghe, L. (2010). Attachment and symptoms of psychopathology: Early maladaptive schemas as a cognitive link? Clinical Psychology and Psychotherapy, 17, 374-385.

Bowlby, J. (1944). Fourty-four juvenile thieves: Their characters and home life. International Journal of Psycho-Analysis, 25, 19-52.

Bregman I. M. \& Wartna, B. S. J. (2010). Recidive TBS 1974-2006: Ontwikkelingen in de strafrechtelijke recidive van ex-terbeschikkinggestelden: Een tussenverslag [Developments in criminal recidivism of former TBS patients: A progress report]. Den Haag: WODC. Factsheet 2010-4.

Broek, E. P. A. van den, Keulen-de Vos, M. E., \& Bernstein, D. P. (2011). Arts therapies and Schema Focused Therapy: A pilot study. The Arts in Psychotherapy, 38, 325-332.

Brinkley, C. A., Newman, J. P., Widiger, T. A., \& Lynam, D. R. (2004). Two approaches to parsing the heterogeneity of psychopathy. Clinical Psychology: Science and Practice, 11, 69-94.

Buschman, J., \& Van Beek, D. (2003). A clinical model for the treatment of personality disordered sexual offenders: An example of theory knitting. Sexual Abuse: A Journal of Research and Treatment, 15, 183-199.

Byrne, B. M. (2001). Structural equation modeling with Amos: Basic concepts, applications and programming. London: Lawrence Erlbaum Associates.

Calvete, E., \& Orue, I. (2011). Social information processing as a mediator between cognitive schemas and aggressive behavior in adolescents. Journal of Abnormal Child Psychology, 40, 105-117.

Calvete, E., Estevez, A., López de Arroyabe, E., \& Ruiz, P. (2005). The schema questionnaire-short form-structure and relationship with automatic thoughts and symptoms of affective disorders. European Journal of Psychological Assessment, 21, 90-99. 
Caspi, A., McClay, J., Moffitt, T. E., Mill, J., Martin, J., Craig I. W., Taylor, A., \& Poulton, R. (2002). Role of genotype in the cycle of violence in maltreated children. Science, 297, 851-854.

Cavadino, M. (1998). Death to the psychopath. The Journal of Forensic Psychiatry, 9, 5-8.

Chakhssi, F., Bernstein, D. P., \& de Ruiter, C. (2012). Early maladaptive schemas in relation to facets of psychopathy and institutional violence in offenders with personality disorders. Legal and Criminological Psychology. Article first published online: 8 November 2012.

Chakhssi, F., de Ruiter, C., \& Bernstein, D. P. (2010a). Reliability and validity of the Dutch version of the Behavioural Status Index: A nurse-rated forensic assessment tool. Assessment, 17, 158-169.

Chakhssi, F., de Ruiter, C., \& Bernstein, D. P. (2010b). Change during forensic treatment in psychopathic versus nonpsychopathic offenders. Journal of Forensic Psychiatry \& Psychology, 21, 660-682.

Chakhssi, F., \& Verwaaijen, A. A. G. (2012). Strafrechtelijke recidive tijdens en na de tbs-behandeling in FPC de Rooyse Wissel: Eerste resultaten [Criminal recidivism during and after treatment in FPC de Rooyse Wissel]. Venray: FPC de Rooyse Wissel.

Christensen, L., \& Mendoza, J. L. (1986). A method of assessing change in a single subject: An alteration of the RC index. Behavior Therapy, 15, 305-308.

Cima, M. (2003). Faking good, bad, and ugly: Malingering in forensic psychiatric inpatients. PhD Thesis. Maastricht: Maastricht University.

Cima, M., Merckelbach, H., Hollnack, S., Butt, C., Kremer, K., Schellbach Matties, R. \& Muris, P. (2003). The other side of malingering: Supernormality. The Clinical Neuropsychologist, 17, 235-243.

Cleckley, H. M. (1941). The mask of sanity: An attempt to reinterpret the so-called psychopathic personality. St. Louis, MO: Mosbey.

Cleckley, H. M. (1988). The mask of sanity: An attempt to clarify some issues about the so called psychopathic personality (5th ed.). Augusta: Emily S. Cleckley.

Cohen, J. (1988). Statistical power analysis for the behavioral sciences (2nd ed.). Hillsdale, NJ: Lawrence Erlbaum.

Coid, J., \& Ullrich, S. (2010). Antisocial personality disorder is on a continuum with psychopathy. Comprehensive psychiatry, 51, 426-433.

Coid, J., Yang, M., Ullrich, S., Roberts, A., \& Hare, R. D. (2009a). Prevalence and correlates of psychopathic traits in the household population of Great Britain. International Journal of Law and Psychiatry, 32, 65-73. 
Coid, J., Yang, M., Ullrich, S., Roberts, A., Moran, P., Bebbington, P., Brugha, T., Jenkins, R., Farrell, M., Lewis, G., Singleton, N., \& Hare, R. D. (2009b). Psychopathy among prisoners in England and Wales. International Journal of Law and Psychiatry, 32, 134-141.

Commissie Visser (2006). Tbs, vandaag over gisteren en morgen [Tbs order, today about yesterday and tommorow]. Den Haag: Sdu Uitgevers.

Cooke D. J., \& Michie C. (2001). Refining the construct of psychopathy: Towards a hierarchical model. Psychological Assessment, 13, 171-188.

Cooke, D. J., Michie, C., \& Hart, S. D. (2006). Facets of psychopathy: Toward clearer measurement. In C. J. Patrick (Ed.), Handbook of psychopathy (pp. 91106). New York, NY: Guilford Press.

Cooke, D. J., Michie, C., Hart, S. D., \& Clark, D. A. (2005). Searching for the pancultural core of psychopathic personality disorder. Personality and Individual Differences, 39, 283-295.

Cooke, D. J., Michie, C., \& Skeem, J. (2007). Understanding the structure of the Psychopathy Checklist-Revised: An exploration of methodological confusion. British Journal of Psychiatry, 190(Suppl. 49), s39 -s50.

D’Silva, K., Duggan, C., \& McCarthy, L. (2004). Does treatment really make psychopaths worse? A review of the evidence. Journal of Personality Disorders, 18, 163-177.

Daversa, M. T. (2010). Early environmental predictors of the affective and interpersonal constructs of psychopathy. International Journal of Offender Therapy and Comparative Criminology, 54, 6-21.

Dawson, D. L., Barnes-Holmes, D., Gresswell, D. M., Hart, A. J., \& Gore, N. J. (2009). Assessing the implicit beliefs of sexual offenders using the implicit relational assessment procedure: A first study. Sexual Abuse: A Journal of Research and Treatment, 21, 57-75.

Dolan, M. C., \& Anderson, I. M., (2003). The relationship between serotonergic function and the Psychopathy Checklist: Screening Version. Journal of Psychopharmacology, 17, 216-222.

Douglas, K. S., Guy, L. S., \& Weir, J. (2006). HCR-20 violence risk assessment scheme: Overview and annotated bibliography. Retrieved May 26, 2008 from http://kdouglas.wordpress.com/hcr-20/.

Douglas, K. S., \& Kropp, P. R. (2002). A prevention-based paradigm for violence risk assessment: Clinical and research applications. Criminal Justice and Behavior, 29, 617-658.

Douglas, K. S., Lilienfeld, S. O., Skeem, J. L., Poythress, N. G., Edens, J. F., \& Patrick, C. J. (2008). Relation of antisocial and psychopathic traits to suiciderelated behavior among offenders. Law and Human Behavior, 32, 511-525. 
Douglas, K. S., \& Skeem, J. L. (2005). Violence risk assessment: Getting specific about being dynamic. Psychology, Public Policy, \& Law, 11, 347-383.

Dowden, C., Antonowicz, D., \& Andrews, D. A. (2003). The effectiveness of relapse prevention with offenders: A meta-analysis. International Journal of Offender Therapy and Comparative Criminology, 47, 516-528.

Dvoskin, J., \& Heilbrun, K. (2001). Risk assessment and release decision-making: Toward resolving the great debate. Journal of the American Academy of Psychiatry and the Law, 29, 6-10.

Edens, J. F., Boccaccini, M. T., \& Johnson, D. W. (2010). Interrater reliability of the PCL-R total and factor scores among psychopathic sex offenders: Are personality features more prone to disagreement than behavioral features? Behavioral Sciences \& the Law, 28, 106-119.

Edens, J. F., Colwell, L. H., Desforges, D. M., \& Fernandez, K. (2005). The impact of mental health evidence on support for capital punishment: Are defendants labeled psychopathic considered more deserving of death? Behavioral sciences \& the law, 235, 603-625.

Emmerik, J. L. van (2001). De Terbeschikkingstelling in maat en getal [The TBS-order in size and numbers]. Den Haag: Ministerie van Justitie, Dienst Justitiële Instellingen.

Erven, A. van. (1999). Behavioural Status Index, Dutch version, (BSI-D). A broad risk assessment for mental health workers. Eindhoven: GGzE.

Fabrigar, L. R., Wegener, D. T., MacCallum, R. C., \& Strahan, E. J. (1999). Evaluating the use of exploratory factor analysis in psychological research. Psychological Methods, 4, 272-299.

Farmer, M., Beech, A. R., \& Ward, T. (2011). Assessing desistance in child molesters: A qualitative analysis. Journal of Interpersonal Violence. Published online before print December 26, 2011.

Farrell, J. M., Shaw, I. A., \& Webber, M. A. (2009). A schema-focused approach to group psychotherapy for outpatients with borderline personality disorder: A randomized controlled trial. Journal of Behavior Therapy and Experimental Psychiatry, 40, 317-328.

Farrington, D. P. (2006). Family background and psychopathy. In C. J. Patrick (Ed.), Handbook of psychopathy (pp. 229-250). New York: Guilford Press.

Fenton, L. R., Cecero, J. J., Nich, C., Frankforter, T. L., \& Carroll, K. M. (2001). Perspective is everything: The predictive validity of working alliance instruments. Journal of Psychotherapy Practice and Research, 10, 262-268.

Finner, H. (1993). On a monotonicity problem in step-down multiple test procedures. Journal of the American Statistical Association, 88, 920-923. 
Finner, H., \& Strassburger, K. (2002). The partitioning principle: a powerful tool in multiple decision theory. Annals of Statistics, 30, 1194-1213.

Frodi, A., Dernevik, M., Sepa, A., Philipson, J., \& Bragesjø, M. (2001). Current attachment representations of incarcerated offenders varying in degree of psychopathy. Attachment and Human Development, 3, 269-283.

Gannon, T. A. (2006). Increasing honest responding on cognitive distortions in child molesters: The bogus pipeline procedure. Journal of Interpersonal Violence, 21, 358-375.

Gannon, T. A., Keown, K., \& Polaschek, D. (2007). Increasing honest responding on cognitive distortions in child molesters: The bogus pipeline revisited. Sexual Abuse: A Journal of Research and Treatment, 19, 5-22.

Gannon, T. A., \& Polaschek, D. L. L. (2005). Do child molesters deliberately fake good on cognitive distortion questionnaires? An information processing-based investigation. Sexual Abuse: A Journal of Research and Treatment, 17, 183200.

Gannon, T. A., \& Polaschek, D. L. L. (2006). Cognitive distortions in child molesters: A re-examination of key theories and research. Clinical Psychology Review, 26, 1000-1019.

Gannon, T. A., Wright, D. B., Beech, A. R., \& Williams, S. (2006). Do child molesters hold distorted beliefs? What does their memory recall tell us? Journal of Sexual Aggression, 12, 5-18.

Giesen-Bloo, J., van Dyck, R., Spinhoven P., van Tilburg W., Dirksen, C., van Asselt, T., Kremers, I., Nadort, M. \& Arntz, A. (2006). Outpatient psychotherapy for borderline personality disorder: A randomized trial of schema focused therapy versus transference focused therapy. Archives of General Psychiatry, 63, 649658.

Goldstein, A. P., Glick, B., Reiner, S., Zimmerman, D., \& Coultry, T. (1987). Aggression Replacement Training. Illinois: Research Press.

Gorsuch, R. L. (1983). Factor analysis ( $2^{\text {nd }}$ ed.). Hillsdale, NJ: Erlbaum.

Graham, N., Kimonis, E. R., Wasserman, A. L., \& Kline, S. M. (2012). Associations among childhood abuse and psychopathy facets in male sexual offenders. Personality Disorders: Theory, Research, and Treatment, 3, 66-75.

Grann, M., Långström, N., Tengström, A., \& Kullgren, G. (1999). Psychopathy (PCL$\mathrm{R})$ predicts violent recidivism among criminal offenders with personality disorders in Sweden. Law and Human Behavior, 23, 205-217.

Gray, N. S., McCulloch, M. J., Smith, J., Morris, M., \& Snowden, R. J. (2003). Violence viewed by psychopathic murderers: Adapting a revealing test may expose those psychopaths who are most likely to kill. Nature, 423, 497-498. 
Guy, S. L., Douglas, K. S., \& Hendry, M. C. (2010). The role of psychopathic personality disorder in violence risk assessments using the HCR-20. Journal of Personality Disorders, 24, 551-580.

Hall, J. R., Benning, S. D., \& Patrick, C. J. (2004). Criterion-related validity of the three-factor model of psychopathy: Personality, behavior, and adaptive functioning. Assessment, 11, 4-16.

Hanson, R. K., \& Morton-Bourgon, K. E. (2004). Predictors of sexual recidivism: An updated meta-analysis. Ottawa: Public Safety Canada.

Hanson, R. K., Harris, A. J. R., Scott, T. L., \& Helmus, L. (2007). Assessing the risk of sexual offenders on community supervision: The Dynamic Supervision Project. Ottawa: Public Safety Canada.

Hare, R. D. (1980). A research scale for the assessment of psychopathy in criminal populations. Personality and Individual Differences, 1, 111-119.

Hare, R. D. (1991). Hare Psychopathy Checklist-Revised (PCL-R). Toronto, Canada: Multi-Health Systems.

Hare. R. D. (1998). The Hare PCL-R: Some issues concerning its use and misuse. Legal and Criminological Psychology, 3, 101-119.

Hare, R. D. (2002). Psychopathy and risk for recidivism and violence. In N. Gray, J. Laing, \& L. Noaks (Eds.), Criminal justice, mental health, and the politics of risk (pp. 27-47). London: Cavendish Publishing.

Hare, R. D. (2003). Hare Psychopathy Checklist-Revised (PCL-R): $2^{\text {nd }}$ edition. Toronto, Canada: Multi-Health Systems.

Hare, R. D., \& Neumann, C. S. (2010). The role of antisociality in the psychopathy construct: Comment on Skeem and Cooke (2010). Psychological Assessment, 22, 446-454.

Hare, R. D., Clark, D., Grann, M., \& Thornton, D. (2000). Psychopathy and the predictive validity of the PCL-R: An international perspective. Behavioral Sciences and the Law. 18, 623-645.

Harpur, T. J., Hare, R. D., \& Hakstian, R. (1989). A two-factor conceptualization of psychopathy: Construct validity and implications for assessment. Psychological Assessment: A Journal of Consulting and Clinical Psychology, 1, 6-17.

Harris, G. T., Rice, M. E., \& Cormier, C. A. (1994). Psychopaths: Is a therapeutic community therapeutic? Therapeutic Communities, 15, 283-300.

Harris, G. T., \& Rice, M. E. (2006). Treatment of Psychopathy: A review of empirical findings in: C. J. Patrick (Ed), Handbook of Psychopathy, (pp. 555-572). New York: Guilford Press. 
Hart, S. D. (2011, November). Understanding and treating clients with psychopathy: A way forward. Paper presented at The 2nd Bergen Conference on the Treatment of Psychopathy, Bergen, Norway.

Hart, S. D., Cox, D., \& Hare, R. D. (1995). Manual for the Psychopathy Checklist: Screening Version (PCL:SV). Toronto, Ontario, Canada: Multi-Health Systems.

Hart, S. D., \& Hare, R. D. (1989). Discriminant validity of the Psychopathy Checklist in a forensic psychiatric population. Psychological Assessment, 1, 211-218.

Heilbrun, K. (1997). Prediction versus management models relevant to risk assessment: The importance of legal decision-making context. Law and Human Behavior, 21, 347-359.

Hemphill, J. F., Hare, R. D., \& Wong, S. C. P. (1998). Psychopathy and recidivism: A review. Legal and Criminological Psychology, 3, 139-170.

Hildebrand, M. (2004). Psychopathy in the treatment of forensic psychiatric patients. Assessment, prevalence, predictive validity and clinical implications. PhD Thesis. Amsterdam: Universiteit van Amsterdam.

Hildebrand, M., Hesper, B. L., Spreen, M., \& Nijman, H. L. I. (2005). De waarde van gestructureerde risicotaxatie en van de diagnose psychopathie: Een onderzoek naar de betrouwbaarheid en predictieve validiteit van de HCR-20, HKT-30 en PCL-R. Amsterdam: Expertisecentrum Forensische Psychiatrie.

Hildebrand, M., \& de Ruiter, C. (2004). PCL-R psychopathy and its relation to DSMIV Axis I and II disorders in a sample of male forensic psychiatric patients in the Netherlands. International Journal of Law and Psychiatry, 27, 233-248.

Hildebrand, M., \& de Ruiter, C. (2010). De strafrechtelijke maatregel terbeschikkingstelling [The judicial measure of detention under a hospital order]. In P.J. van Koppen, H. Merckelbach, M. Jelicic \& J. De Keijser (Eds.), Reizen met mijn rechter (pp. 1021-1042). Deventer: Kluwer.

Hildebrand, M., \& de Ruiter, C. (2012). Psychopathic traits and change on indicators of dynamic risk factors during inpatient forensic psychiatric treatment. International Journal of Law and Psychiatry, 35, 276-288.

Hildebrand, M., de Ruiter, C., \& de Vogel, V. (2004). Psychopathy and sexual deviance in treated rapists: Association with sexual and nonsexual recidivism. Sexual Abuse: A Journal of Research and Treatment, 16, 1-24.

Hildebrand, M., de Ruiter, C., \& Nijman, H. L. I. (2004). PCL-R psychopathy predicts disruptive behavior among male offenders in a Dutch forensic psychiatric hospital. Journal of Interpersonal Violence, 19, 13-29.

Hobson, J., Shine, J., \& Roberts, R. (2000). How do psychopaths behave in a prison therapeutic community? Psychology, Crime and Law, 6, 139-154. 
Hollin, C. R., \& Palmer, E. J. (2006) Offending behaviour programmes: Development, application, and controversies. Chichester: John Wiley \& Sons.

Hornsveld, R. H. J., Nijman, H. L. I., Hollin, C. R., \& Kraaimaat, F. W. (2007). Development of the Observation Scale for Aggressive Behavior (OSAB) for Dutch forensic psychiatric inpatients with an antisocial personality disorder. International Journal of Law and Psychiatry, 30, 480-491.

Horvath, A. O., \& Symonds, B. D. (1991). Relation between working alliance and outcome in psychotherapy: A meta-analysis. Journal of Counseling Psychology, 38, 139-149.

Hu, L. T., \& Bentler, P. (1999). Cutoff criteria for fit indexes in covariance structure analysis: Conventional criteria versus new alternatives. Structural Equation Modeling, 6, 1-55.

Huizinga, D., Haberstick, B., Smolen, A., Menard, S., Young, S., Corley, R., Stallings, M. C., Grotpeter, J., \& Hewitt, J. K. (2006). Childhood maltreatment, subsequent antisocial behavior, and the role of monoamine oxidase $A$ genotype. Biological Psychiatry, 60, 677-683.

IJzendoorn, M. H. van, Feldbrugge, J., Derks, F. C. H., de Ruiter, C., Verhagen M. F., Philipse, M. W. G., van der Staak, C. P., \& Riksen-Walraven, J. M. (1997). Attachment representations of personality-disordered criminal offenders. American Journal of Orthopsychiatry, 67, 449-459.

Jacobson, N. S., \& Truax, P. (1991). Clinical significance: A statistical approach to defining meaningful change in psychotherapy research. Journal of Consulting and Clinical Psychology, 59, 12-19.

James, L. R., Demaree, R. G., \& Wolf, G. (1984). Estimating within-group interrater reliability with and without response bias. Journal of Applied Psychology, 69, 85-98.

Jespersen, A. F., Lalumière, M. L., \& Seto, M. C. (2009). Sexual abuse history among adult sex offenders and non-sex offenders: A meta-analysis. Child Abuse \& Neglect, 33, 179-192.

Jong, C. A. J. de, Derks, F. C. H., van Oel, C. J., \& Rinne, T. (1996). Gestructureerd Interview voor de DSM-IV Persoonlijkheidsstoornissen. Sint Oedenrode: Stichting Verslavingszorg Oost Brabant.

Jovev, M., \& Jackson, H. J. (2004). Early maladaptive schemas in personality disordered individuals. Journal of Personality Disorders, 18, 467-478.

Kellogg, S. H., \& Young, J. E. (2006). Schema therapy for borderline personality disorder. Journal of Clinical Psychology, 62, 445-458.

Kennealy, P. J., Skeem, J. L., Walters, G. D., \& Camp, J. (2010). Do core interpersonal and affective traits of PCL-R Psychopathy interact with antisocial 
behavior and disinhibition to predict violence? Psychological Assessment, 22, 569-580.

Keown, K., Gannon, T. A., \& Ward, T. (2010). What's in a measure? A multi-method study of child sexual offenders ' beliefs. Psychology, Crime \& Law, 16, 125-143.

Kernberg, O. F., Yeomans, F. E., Clarkin, J. F., \& Levy, K. N. (2008). Transference focused psychotherapy: Overview and update. The International Journal of Psychoanalysis, 89, 601-620.

Keulen-de Vos, M., Bernstein, D. P., Vanstipelen, S., de Vogel, V., Lucker, T., \& Arntz, A. (2012). Emotional states in criminal behavior. Manuscript submitted for publication.

Kiehl, K. A., Smith, A. M., Hare, R. D., Forster, B. B., Brink, J., \& Liddle, P. F. (2001). Limbic abnormalities in affective processing by criminal psychopaths as revealed by functional magnetic resonance imaging. Biological Psychiatry, $50,677-684$.

Kogel, C. H. de, Verwers, C., \& den Hartogh, V. E. (2005). 'Blijvend delictgevaarlijk' - empirische schattingen en conceptuele verheldering [Onderzoek \& Beleid, 226]. Den Haag: Boom Juridische uitgevers.

Koivisto, H., \& Haapasalo, J. (1996). Childhood maltreatment and adulthood psychopathy in light of file-based assessments among mental state examinees. Studies on Crime and Crime Prevention, 5, 91-104.

Lang, S., af Klinteberg, B., \& Alm, P. O. (2002). Adult psychopathy and violent behavior in males with early neglect and abuse. Acta Psychiatrica Scandinavica, 106, 93-100.

Lansford, J. E., Miller-Johnson, S., Berlin, L. J., Dodge, K. A., Bates, J. E., \& Pettit, G. S. (2007). Early physical abuse and later violent delinquency: A prospective longitudinal study. Child Maltreatment, 12, 233-245.

Laws, D. R. (1999). Relapse prevention: The state of the art. Journal of Interpersonal Violence, 14, 285-302.

Laws, D. R., Hudson, S. M., \& Ward, T. (2000). Remaking relapse prevention with sex offenders: A sourcebook. Thousand Oaks: Sage.

Lee, C. W., Taylor, G., \& Dunn, J. (1999). Factor structure of the Schema Questionnaire in a large clinical sample. Cognitive Therapy \& Research, 23, 441-451.

Leeuwen, M. L. van, Baaren, R. B. van, Chakhssi, F., Loonen, M. G. M., Lippman, M., \& Dijksterhuis, A. (2013). Assessment of implicit sexual associations in non-incarcerated pedophiles. Archives of Sexual Behavior. Article first published online: 24 april 2013. 
Leistico, A. R., Salekin, R. T., DeCoster, J., \& Rogers, R. (2008). A large-scale metaanalysis relating the Hare measures of psychopathy to antisocial conduct. Law and Human Behavior, 32, 28-45.

Liberman, R. P., DeRisi, W. J., \& Mueser, K. T. (1989). Social skills training for psychiatric patients. New York: Pergamon Press.

Lilienfeld, S. O. (1994). Conceptual problems in the assessment of psychopathy. Clinical Psychology Review, 14, 17-38.

Lobbestael, J., \& Arntz, A. (2010). Emotional, cognitive and physiological correlates of abuse-related stress in borderline and antisocial personality disorder.

Behaviour Research and Therapy, 48, 116-124.

Lobbestael, J., \& Arntz, A. (2012). The state dependency of cognitive schemas in antisocial patients. Psychiatry Research, 198, 452-456.

Lobbestael, J., Arntz, A., Cima, M., \& Chakhssi, F. (2009). Effects of induced anger in patients with antisocial personality disorder. Psychological Medicine, 39, 557-568.

Lobbestael, J., Arntz, A., Harkema-Schouten, P., \& Bernstein, D. (2009)

Development and psychometric evaluation of a new assessment method for childhood maltreatment experiences: The interview for traumatic events in children (ITEC). Child Abuse \& Neglect, 2009, 505-517.

Lobbestael, J., van Vreeswijk, M., Spinhoven, P., Schouten, E., \& Arntz, A. (2010). Reliability and validity of the short Schema Mode Inventory (SMI). Behavioural and Cognitive Psychotherapy, 38, 437-458.

Looman, J. (1995). Sexual fantasies of child molesters. Canadian Journal of Behavioural Science, 27, 321-332.

Lösel, F. (1995). Management of psychopaths. Issues in Criminological and Legal Psychology, 24, 100-106.

Lösel, F., \& Schmucker, M. (2005). The effectiveness of treatment for sexual offenders: A comprehensive meta-analysis. Journal of Experimental Criminology, 1, 117-146.

Luntz, B. K., \& Widom, C. S. (1994). Antisocial personality disorders in abused and neglected children grown up. American Journal of Psychiatry, 151, 670-674.

Lynam, D. R., \& Gudonis, L. (2005). The development of psychopathy. Annual Review of Clinical Psychology, 1, 381-407.

MacCallum, R. C., Widaman, K. F., Zhang, S., \& Hong, S. (1999). Sample size in factor analysis. Psychological Methods, 4, 84-99. 
Mahgoub, N.A. (1988). 'Bridging' therapy in hospital and community-based psychiatric care: a comparative study. PhD thesis. Sheffield: Sheffield City Library.

Mandeville-Norden, R., Beech, A., \& Hayes, E. (2008). Examining the effectiveness of a UK community-based sexual offender treatment programme for child abusers. Psychology, Crime \& Law, 14, 493-512.

Mann, R., \& Beech, A. (2003). Cognitive distortions, schemas, and implicit theories. In T.Ward, D. R. Laws, \& S. M. Hudson (Eds.), Sexual deviance: Issues and controversies (pp. 135-153). London: Sage.

Marshall, L. A., \& Cooke, D. J. (1999). The childhood experiences of psychopaths: A retrospective study of familial and societal factors. Journal of Personality Disorders, 13, 211-225.

Marshall, W. L., Marshall, L. E., \& Kingston, D. A. (2011). Are the cognitive distortions of child molesters in need of treatment? Journal of Sexual Aggression, 17, 118-129.

Marshall, W. L., Marshall, L. E., Serran, G. A., \& O’Brien, M. D. (2011). Rehabilitating sexual offenders: A strengths based approach. Washington, DC: American Psychological Association.

Marshall, W. L., Serran, G. A., \& Cortoni, F. A. (2000). Childhood attachments, sexual abuse, and their relationship to adult coping in child molesters. Sexual Abuse: A Journal of Research and Treatment, 12, 17-26.

Maruna, S., \& Mann, R. E. (2006). A fundamental attribution error? Rethinking cognitive distortions. Legal and Criminological Psychology, 11, 155-177.

Marziano, V., Ward, T., Beech, A. R., \& Pattison, P. (2006). Identification of five fundamental implicit theories underlying cognitive distortions in child abusers: A preliminary study. Psychology, Crime \& Law, 12, 97-105.

Maxfield, M. G., \& Widom, C. S. (1996). The cycle of violence: Revisited six years later. Archives of Pediatric and Adolescent Medicine, 150, 390-395.

McGlinchey, J. B., Atkins, D. C., \& Jacobson, N. S. (2002). Clinical significance methods: Which one to use and how useful are they? Behavior Therapy, 33, 529-550.

McKibben, A., Proulx, J., \& Lusignan, R. (1994). Relationships between conflict, affect and deviant sexual behaviors in rapists and pedophiles: The assessment and treatment of sex offenders. Behaviour Research and Therapy, $32,571-575$.

Meehl, P. E. (1945). A simple algebraic development of Horst's suppressor variables. American Journal of Psychology, 58, 550-554. 
Miller, A. K., Rufino, K. A., Boccaccini, M. T., Jackson, R., \& Murrie, D. C. (2011). On individual differences in person perception: Raters' personality traits relate to their Psychopathy Checklist-Revised scoring tendencies. Assessment, 18, 253-260.

Miller, W. R., \& Rollnick, S. (2002). Motivational interviewing: Preparing people to change. New York: Guilford Press.

Milner, J. R., \& Webster, D. S. (2005). Identifying schemas in child Molesters, rapists and violent offenders. Sexual Abuse: A Journal of Research and Treatment, 17, 425-439.

Ministerie van Justitie (2009). Beleidskader Longstay Forensische Zorg [Policy on Longstay Forensic Treatment]. Den Haag: Directie Forensische Zorg.

Morrissey, C., Mooney, P., Hogue, T. E., Lindsay, W. R., \& Taylor, J. L. (2007). Predictive validity of the PCL-R for offenders with intellectual disability in a high security hospital: Treatment progress. Journal of Intellectual \& Developmental Disability, 32, 125-133.

Murphy, J. M. (1976). Psychiatric labeling in cross-cultural perspective. Science, $191,1019-1027$.

Murrie, D. C., Boccaccini, M. T., Johnson, J., \& Janke, C. (2008). Does interrater (dis)agreement on Psychopathy Checklist scores in sexually violent predator trials suggest partisan allegiance in forensic evaluation? Law and Human Behavior, 32, 352-362.

Murrie, D. C., Boccaccini, M. T., Turner, D. B., Woods, C., Tussey, C., \& Meeks, M. (2009). Rater (dis)agreement on risk assessment measure in sexually violent predator proceedings: Evidence of adversarial allegiance in forensic evaluation? Psychology, Public Policy and Law, 15, 19-53.

Myers, D., \& Hayes, J. A. (2006). Effects of therapist general self-disclosure and countertransference disclosure on ratings of the therapist and session.

Psychotherapy: Theory, Research, Practice, Training, 43, 173-185.

Nadort, M., Arntz, A., Smit, J. H., Giesen-Bloo, J., Eikelenboom, M., Spinhoven, P., van Asselt, T., Wensing, M., \& van Dyck, R. (2009). Implementation of outpatient schema therapy for borderline personality disorder with versus without crisis support of the therapist outside office hours: A randomized trial. Behaviour Research and Therapy, 47, 961-973.

Nagtegaal, M. H., Horst, R. P. van der, \& Schönberger, H. J. M. (2011). Inzicht in de verblijfsduur van tbs-gestelden: Cijfers en mogelijke verklaringen. Den Haag: Boom Juridische uitgevers.

Nederlands Instituut voor Psychologen [Dutch Association of Psychologists] (2010). Algemeen standard testgebruik. Amsterdam: NIP. 
Neumann, C. S., \& Hare, R. D. (2008). Psychopathic traits in a large community sample: Links to violence, alcohol use, and intelligence. Journal of Consulting and Clinical Psychology, 76, 893-899.

Neumann, C. S., Hare, R. D., \& Newman, J. P. (2007). The super-ordinate nature of the Psychopathy Checklist-Revised. Journal of Personality Disorders, 21, $102-$ 117.

Newman, J. P. (1998). Psychopathic behavior: An information processing perspective. In D. J. Cooke, R. D. Hare, \& A. Forth (Eds.), Psychopathy: Theory, Research and Implications for Society. The Netherlands: Kluwer Academic Publishers, (pp. 81-104).

Nicholls, T. L., Brink, J., Desmarais, S. L., Webster, C. D., \& Martin, M. L. (2006). The Short-Term Assessment of Risk and Treatability (START): A prospective validation study in a forensic psychiatric sample. Assessment, 13, 313-327.

Nijman, H. L. I., Evers, C., Merckelbach, H. L. G. J., \& Palmstierna, T. (2002). Assessing aggression severity with the revised Staff Observation Aggression Scale (SOAS-R). Journal of Nervous and Mental Disease, 190, 198 - 200.

Nordahl, H. M., \& Nysaeter, T. E. (2005). Schema therapy for patients with borderline personality disorder: A single case series. Journal of Behavior Therapy and Experimental Psychiatry, 36, 254-264.

Nunnally, J. C. (1967). Psychometric theory. New York: McGraw-Hill.

Ó Ciardha, C., \& Gannon, T. A. (2011). The cognitive distortions of child molesters are in need of treatment. Journal of Sexual Aggression, 17, 130-141.

Ogles, B. M., Lunnen, K. M., \& Bonesteel, K. (2001). Clinical significance: History, application, and current practice. Clinical Psychology Review, 21, 421-446.

Ogloff, J. R. P. (2006). Psychopathy/antisocial personality disorder conundrum. Australian and New Zealand Journal of Psychiatry, 40, 519-528.

Ogloff, J. R. P., Wong, S. C., \& Greenwood, A. (1990). Treating criminal psychopaths in a therapeutic community program. Behavioral Sciences and the Law, 8, 181190.

Olejnik, S. F., \& Algina, J. (1984). Parametic ANCOVA and the rank transform ANCOVA when the data are conditionally non-normal and heteroscedastic. Journal of Educational Statistics, 9, 129-149.

Olver, M. E. \& Wong, S. C. (2006). Psychopathy, sexual deviance, and recidivism among sex offenders. Sexual Abuse: A Journal of Research and Treatment 18, 65-82.

Olver, M. E., \& Wong, S. C. (2009). Therapeutic responses of psychopathic sexual offenders: Treatment attrition, therapeutic change, and long term recidivism. Journal of Consulting and Clinical Psychology, 77, 328-336. 
Patrick, C.J. (Ed.). (2006). Handbook of Psychopathy. New York: Guilford Press.

Paulhus, D. L., Robins, R. W., Trzesniewski, K. H., \& Tracy, J. L. (2004). Two replicable suppressor situations in personality research. Multivariate Behavioral Research, 39, 303-329.

Pfohl, B., Blum, N., \& Zimmerman, M. (1997). Structured interview for DSM-IV personality: SIDP-IV. Washington, DC: American Psychiatric Press.

Porter, S., Fairweather, D., Drugge, J., Birt, A., Hervé, H., \& Boer, D. (2000). Profiles of psychopathy in incarcerated sexual offenders. Criminal Justice and Behavior, 27, 216-233.

Poythress, N. G., Skeem, J. L., \& Lilienfeld, S. O. (2006). Associations among early abuse, dissociation, and psychopathy in an offender sample. Journal of Abnormal Psychology, 115, 288-297.

Proulx, J., McKibben, A., \& Lusignan, R. (1996). Relationships between affective components and sexual behaviours in sexual aggressors. Sexual Abuse: $A$ Journal of Research and Treatment, 8, 279-290.

Quinta Gomes, L., \& Nobre, P. (2012). Early maladaptive schemas and sexual dysfunction in men. Archives of Sexual Behavior, 41, 311-320.

Raad voor Strafrechtstoepassing en Jeugdbescherming (2008). Advies Longstay. Retrieved from www.rsj.nl/Images/bw-rsj-longstay_tcm60-102540.pdf

Rafaeli, E., Bernstein, D. P., \& Young, J. E. (2011). Schematherapy: Distinctive features. New York: Routledge.

Raine A., Lencz T., Bihrle S., LaCasse L., \& Colletti P. (2000). Reduced prefrontal gray matter volume and reduced autonomic activity in antisocial personality disorder. Archives of General Psychiatry, 57,119-127.

Reed, V., Woods, P., \& Robinson, D. (2000). Behavioural Status Index (BEST-Index): A 'life skills' assessment for selecting and monitoring therapy in mental health care. UK: Psychometric Press.

Reid, W. H., \& Gacono, C. B. (2000). Treatment of antisocial personality, psychopathy and other characterologic antisocial syndromes. Behavioural Science and the Law, 18, 647-662.

Rice, M. E., \& Harris, G. T. (1997). Cross-validation and extension of the violence risk appraisal guide for child molesters and rapists. Law and Human Behavior, 21, 231-241.

Rice, M. E., Harris, G. T., \& Cormier, C. A. (1992). An evaluation of a maximum security therapeutic community for psychopaths and other mentally disordered offenders. Law and Human Behavior, 16, 399-412. 
Richardson, G. (2005). Early maladaptive schemas in a sample of British adolescent sexual abusers: Implications for therapy. Journal of Sexual Aggression, 11, 259-276.

Rijkeboer, M. M., \& Huntjens, R. J. C. (2007, July). EMS's in forensic settings: An implicit measure of insufficient self-control/self-discipline: A pilot study. Paper presented at the World Congress of Behavioural \& Cognitive Therapies, Barcelona.

Rijkeboer, M. M., \& van den Bergh, H. (2006). Multiple group confirmatory factor analysis of the Young Schema Questionnaire in a Dutch clinical versus nonclinical population. Cognitive Therapy and Research, 30, 263-278.

Rijkeboer, M. M., van den Bergh, H., \& van den Bout, J. (2005). Stability and discriminative power of the Young Schema Questionnaire in a Dutch clinical versus nonclinical population. Journal of Behavior Therapy and Experimental Psychiatry, 36, 129-144.

Robinson D., Reed V., \& Lange A. (1996). Developing risk assessment scales in forensic psychiatric care. Psychiatric Care, 3, 146-152.

Roelofs, J., Lee, C., Ruijten, T., \& Lobbestael, J. (2011). The mediating role of early maladaptive schemas in the relation between quality of attachment relationships and symptoms of depression in adolescents. Behavioural and Cognitive Psychotherapy, 39, 471-479.

Rogers, R. (Ed.). (1997). Clinical assessment of malingering and deception (2nd ed.). New York: Guilford Press.

Rogers, R. (Ed). (2008). Clinical assessment of malingering and deception (3rd Ed.). New York: Guilford Press.

Roper, L., Dickson, J., Tinwell, C., Booth, P., \& McGuire, J. (2010). Maladaptive cognitive schemas in alcohol dependence: Changes associated with a brief residential abstinence program. Cognitive Therapy and Research, 34, 207-215.

Ross, T., Woods, P., Reed, V., Sookoo, S., Dean, A., Kettles, A. M., Almvik, R., Ter Horst, P., Brown, I., Collins, M., Walker, H., \& Pfäfflin, F. (2008). Selecting and monitoring living skills in forensic mental health care: Cross-border validation of the BEST-Index. International Journal of Mental Health, 36, 3-16.

Ruiter, C. de, \& Hildebrand, M. (2003). The dual nature of forensic psychiatric practice: Risk assessment and management under the Dutch TBS-order. In P. J. van Koppen \& S.D. Penrod (Eds.), Adversary vs. inquisitorial justice: Psychological perspectives on criminal justice systems (pp. 91-106). New York: Plenum.

Ruiter, C. de, \& Trestman, R. L. (2007). Prevalence and treatment of personality disorders in Dutch forensic mental health services. Journal of the American Academy of Psychiatry and the Law, 35, 92-97. 
Salekin, R. T. (2002). Psychopathy and therapeutic pessimism: Clinical lore or clinical reality? Clinical Psychology Review, 22, 79-112.

Salekin, R. T., Worley, C., \& Grimes, R. D. (2010). Treatment of psychopathy: A review and brief introduction to the mental model approach for psychopathy. Behavioral Sciences and the Law, 28, 235-266.

Salter, D., McMillan, D., Richards, M., Talbot, T., Hodges, J., Bentovim, A., Hastings, R., Stevenson, J., \& Skuse, D. (2003). Development of sexually abusive behavior in sexually victimized males: A longitudinal study. The Lancet, 361, 471-476.

Schmidt, N. B., Joiner, T. E., Young, J. E., \& Telch, M. J. (1995). The schema questionnaire: Investigation of psychometric properties and the hierarchical structure of a measure of maladaptive schemas. Cognitive Therapy and Research, 19, 295-321.

Seto, M. C., \& Barbaree, H.E. (1999). Psychopathy, treatment behavior, and sex offenders recidivism. Journal of Interpersonal Violence, 14, 1235-1248.

Seto, M. C., \& Lalumière, M. L. (2010). What is so special about male adolescent sex offending? A review and test of explanations through meta-analysis. Psychological Bulletin, 136, 526-575.

Shrout, P. E., \& Fleiss, J. L. (1979). Intraclass Correlations: Uses in assessing rater reliability. Psychological Bulletin, 86, 420-428.

Sigre-Leirós, V. L., Carvalho, J., \& Nobre, P. (2012). Early maladaptive schemas and aggressive sexual behavior: A preliminary study with male college students. Journal of Sexual Medicine. Article first published online: 20 AUG 2012.

Skeem, J. L. (2008, July). High risk, not hopeless: Recent research on treating individuals with psychopathy. Paper presented at the annual meeting of the International Association of Forensic Mental Health: Vienna, Austria.

Skeem, J. L., \& Cooke, D. J. (2010). Is criminal behavior a central component of psychopathy? Conceptual directions for resolving the debate. Psychological Assessment, 22, 433-445.

Skeem, J. L., Monahan, J., \& Mulvey, E. (2002). Psychopathy, treatment involvement, and subsequent violence among civil psychiatric patients. Law and Human Behavior, 26, 577-603.

Skeem, J. L., Polaschek, D. L. L., Patrick, C. J., \& Lilienfeld, S. O. (2011). Psychopathic personality: Bridging the gap between empirical evidence and public policy. Psychological Science in the Public Interest, 12, 95-162.

Smeijsters, H., \& Cleven, G. (2006). The treatment of aggression using arts therapies in forensic psychiatry: Results of a qualitative inquiry. The Arts in Psychotherapy, 33, 37-58. 
Smith, C., \& Thornberry, T. (1995). The relationship between childhood maltreatment and adolescent involvement in delinquency. Criminology, 33, 451-481.

Spinhoven, P., Giesen-Bloo, J., van Dyck, R., Kooiman, K., \& Arntz, A. (2007). The therapeutic alliance in schema-focused therapy and transference-focused psychotherapy for borderline personality disorder. Journal of Consulting and Clinical Psychology, 75, 104-115.

SPSS Inc. (2004). SPSS Complex Samples 13.0. [Computer software]. Chicago, IL: SPSS Inc.

SPSS Inc. (2005). SPSS 13.0 for Windows. [Computer software]. Chicago, IL: SPSS Inc.

Sterk, F., \& Rijkeboer, M. M. (1997). Schema-Vragenlijst [Schema-Questionnaire]. Utrecht, The Netherlands: Ambulatorium Utrecht University.

Stopa, L., Thorne, P., Waters, A., \& Preston, J. (2001). Are the long and short forms of the Young Schema Questionnaire comparable and how well does each version predict psychopathology scores? Journal of Cognitive Therapy: An International Quarterly, 15, 253-272.

Strand, S., \& Belfrage, H. (2001). Comparison of HCR-20 scores in violent mentally disordered men and women: Gender differences and similarities. Psychology, Crime and Law, 7, 71-79.

Tangney, J. P., \& Stuewig, J. (2004). A moral emotional perspective on evil persons and evil deeds. In A. Miller (Ed.), The social psychology of good and evil: Understanding our capacity for kindness and cruelty (pp. 327-355). New York: Guilford Press.

Thornberry, T. P., Ireland, T. O., \& Smith, C. A. (2001). The importance of timing: The varying impact of childhood and adolescent maltreatment on multiple problem outcomes. Development and Psychopathology, 13, 957-979.

Thornton, D., Beech, A. R., \& Marshall, W. L. (2004). Pretreatment self-esteem and posttreatment sexual recidivism. International Journal of Offender Therapy and Comparative Criminology, 48, 587-599.

Tichenor, V., \& Hill, C. E. (1989). A comparison of six measures of working alliance. Psychotherapy: Theory, Research, Practice, Training, 26, 195-199.

Tiihonen, J., Rossi, R., Laskso, M. P., Hodgins, S., Testa, C., Perez, J., RepoTiihonen E., Vaurio, O., Soininein, H., Aronen, H.J., Kononen, M., Thompson, P., \& Frisoni, G. B. (2008). Brain anatomy of persistent violent offenders: More rather than less. Psychiatry Research: Neuroimaging, 163, 201-212.

Timmerman, I. G., Vastenburg, N. C., \& Emmelkamp, P. M. G. (2001). The Forensic Inpatient Observation Scale (FIOS): Development, reliability and validity.

Criminal Behavior and Mental Health, 11, 144-162. 
Tracey, T. J., \& Kokotovic, A. M. (1989). Factor structure of the Working Alliance Inventory. Psychological Assessment, 1, 207-210.

Tremblay, P. F., \& Dozois, D. J. A. (2009). Another perspective on trait aggressiveness: Maladaptive schemas. Personality and Individual Differences, 46, 569-574.

Verona, E., Patrick, C. J., \& Joiner, T. E. (2001). Psychopathy, antisocial personality, and suicide risk. Journal of Abnormal Psychology, 110, 462-470.

Vertommen, H., Verheul, R., de Ruiter, C., \& Hildebrand, M. (2002). Hare's Psychopathie Checklist: Handleiding [Dutch version of the Hare Psychopathy Checklist-Revised: Manual]. Amsterdam: Harcourt / Pearson.

Viding, E., Blair, R. J. R., Moffitt, T. E., \& Plomin, R. (2005). Evidence for substantial genetic risk for psychopathy in 7-year-olds. Journal of Child Psychology and Psychiatry, 46, 592-597.

Vogel, V. de., \& de Ruiter, C. (2006). Structured professional judgment of violence risk in forensic clinical practice: A prospective study into the predictive validity of the Dutch HCR-20. Psychology, Crime and Law, 12, 321-336.

Vogel, V. de., de Ruiter, C., Hildebrand, M., Bos, B., \& van de Ven, P. (2004). Type of discharge and risk of recidivism measured by the HCR-20: A retrospective study in a Dutch sample of treated forensic psychiatric patients. International Journal of Forensic Mental Health, 3, 149-165.

Waller, G., Meyer, C., \& Ohanian, V. (2001). Psychometric properties of the long and short versions of the Young Schema Questionnaire: Core beliefs among bulimic and comparison women. Cognitive Therapy and Research, 25, 137-147.

Walsh, Z., Swogger, M. T., \& Kosson, D. S. (2009). Psychopathy and instrumental violence: Facet level relationships. Journal of Personality Disorders, 23, 416424.

Walters, G. D., \& Heilbrun, K. (2010). Violence risk assessment and Facet 4 of the Psychopathy Checklist: Predicting institutional and community aggression in two forensic samples. Assessment, 17, 259-268.

Walters, G. D., Knight, R. A., Grann, M., \& Dahle, K. P. (2008). Incremental validity of the Psychopathy Checklist facet scores: Predicting release outcome in six samples. Journal of Abnormal Psychology, 117, 396-405.

Ward, T. (2000). Sexual offenders“ cognitive distortions as implicit theories. Aggression and Violent Behavior, 5, 491-507.

Ward, T., \& Keenan, T. (1999). Child molesters' implicit theories. Journal of Interpersonal Violence, 14, 821-838. 
Ward, T., Gannon, T. A., \& Keown, K. (2006). Beliefs, values, and action: The judgment model of cognitive distortions. Aggression and Violent Behavior, 11, 323-340.

Ward, T., Hudson, S. M, Johnston, L., \& Marshall, W. L. (1997). Cognitive distortions in sex offenders: An integrative review. Clinical Psychology Review, 17, 479-507.

Wartna, B. S. J., Harbachi, S. el, \& Essers, A. A. M. (2006). Strafrechtelijke recidive ex-terbeschikkinggestelden: Fact sheet 2006-8 [Criminal recidivism of ex-forensic psychiatric patients detained under the Dutch TBS-order]. Den Haag: WODC

Webster, C. D., Douglas, K. S., Eaves, D., \& Hart, S. D. (1997). HCR-20. Assessing risk for violence, version 2. Burnaby, BC: Simon Fraser University, Mental Health, Law and Policy Institute.

Wechsler, D. (2001). WAIS-III Nederlandstalige bewerking: Technische handleiding [WAIS-III Dutch adaptation: Technical Manual]. Lisse: Swets Test Publishers.

Weiler, B. L., \& Widom, C. S. (1996). Psychopathy and violent behaviour in abused and neglected young adults. Criminal Behaviour and Mental Health, 6, 253271.

Weisman, R. (1995). Reflections on the Oakridge experiment with mentally disordered offenders, 1965-1968. International Journal of Law and Psychiatry, 18, 265-290.

Weizmann-Henelius, G., Grönroos, M., Putkonen, H., Eronen, M., Lindberg, N., \& Häkkänen-Nyholm, H. (2010). Psychopathy and gender differences in childhood psychosocial characteristics in homicide offenders - a nationwide registerbased study. Journal of Forensic Psychiatry \& Psychology, 6, 801-814.

Whitaker, D. J., Le, B., Hanson, R. K., Baker, C. K., McMahon, P., Ryan, G., Klein, A., \& Rice, D. D. (2008). Risk factors for the perpetration of child sexual abuse: A review and meta-analysis. Child Abuse \& Neglect, 32, 529-548.

Widom, C. S. \& Ames, M. (1994) Criminal consequences of childhood sexual victimization. Child Abuse and Neglect, 18, 303-318.

Wong, S. C., \& Hare, R. D. (2005). Guidelines for a psychopathy treatment program. Toronto: Multi-Health Systems.

Woods, P. (2000). The Behavioural Status Index: Descriptive studies within a forensic context. PhD Thesis. Anglia Polytechnic University.

Woods, P., Reed, V., \& Collins, M. (2001a). Measuring insight in a high security forensic setting using the Behavioural Status Index. British Journal of Forensic Practice, 3, 3-12. 
Woods, P., Reed, V., \& Collins, M. (2001b). Measuring risk in a high security forensic setting using the Behavioural Status Index. International Journal of Psychiatric Nursing Research, 7, 793-805.

Woods, P., Reed, V., \& Collins, M. (2001c). Measuring communication and social skills in a high security forensic setting using the Behavioural Status Index. International Journal of Psychiatric Nursing Research, 7, 761-777.

Woods, P., Reed, V., \& Collins, M. (2003a). Relationships between risk and insight in a high security forensic setting. Journal of Psychiatric and Mental Health Nursing, 10, 510-517.

Woods, P., Reed, V., \& Collins, M. (2003b). Exploring core relationships between insight, and communication and social skills in mentally disordered offenders. Journal of Psychiatric and Mental Health Nursing, 10, 518-525.

Woods, P., Reed, V., \& Collins, M. (2004). Relationships between risk and communication and social skills in a high security forensic setting. Issues in Mental Health Nursing, 8, 769-782.

Woods, P., Reed, V., \& Collins, M. (2005). The Behavioural Status Index: Testing a social risk assessment model in a high security forensic setting. Journal of Forensic Nursing, 1, 9-19.

Woods, P., Reed, V., \& Robinson, D. (1999). The Behavioural Status Index: Therapeutic assessment of risk, insight, communication and social skills. Journal of Psychiatric and Mental Health Nursing, 6, 79-90.

Yang, Y., \& Raine, A. (2009). Prefrontal structural and functional brain imaging findings in antisocial, violent, and psychopathic individuals: A meta-analysis. Psychiatry Research, 174, 81-88.

Young, J. E. (1994). Cognitive therapy for personality disorders: A schema-focused approach (2nd ed.). Sarasota, FL: Professional Resource Press.

Young, J. E., \& Brown, G. (1994). Young Schema Questionnaire (2nd ed.). New York: Cognitive Therapy Center of New York.

Young, J. E., \& Fosse, G. (2005). Schema Therapy Rating Scale. New York: Cognitive Therapy Center of New York.

Young, J. E., Klosko, J. S., \& Weishaar, M. E. (2003). Schema therapy: A practitioner's guide. New York: Guilford Press.

Young, J. E., \& Pijnaker, H. (1999). Cognitieve therapie voor persoonlijkheidsstoornissen: een schemagerichte benadering. Houten: Bohn Stafleu Van Loghum.

Yudofsky, S. C., Silver, J. M., Jackson, W., Endicott, J., \& Williams, D. (1986). The Overt Aggression Scale for the objective rating of verbal and physical aggression. American Journal of Psychiatry, 143, 35-39. 
DANKWOORD

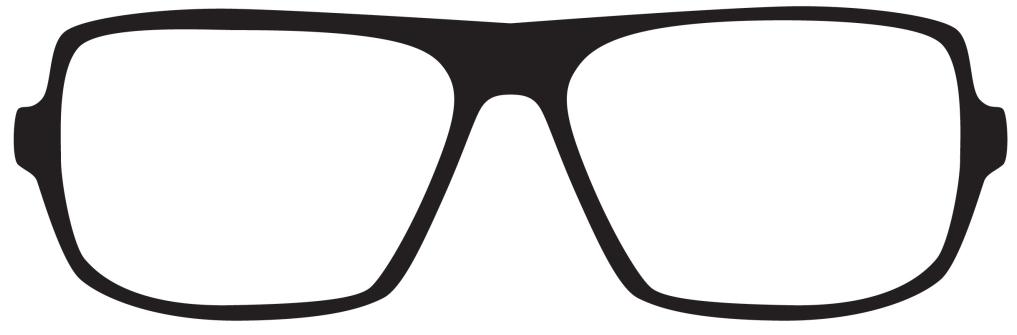

$\circ \circ$ 
De afgelopen jaren heb ik met veel plezier gewerkt aan het onderzoek dat heeft geleid tot dit proefschrift. Ik wil iedereen bedanken waarmee ik in de afgelopen jaren heb samengewerkt; dat er zijn teveel om op te noemen. In dit dankwoord zal ik me dan beperken tot de mensen die een belangrijke bijdrage hebben geleverd aan dit proefschrift.

Allereerst dank ik mijn promotoren, de professoren Corine de Ruiter en David Bernstein voor het vertrouwen om een promotie-onderzoek onder jullie begeleiding te mogen uitvoeren. Het was mij een eer om jullie student te zijn!

Beste Corine, ik heb veel respect en bewondering voor de passie en integriteit waarmee jij je vak uitoefent en hoe je vanuit je hart je kennis deelt met anderen. Ik heb veel geleerd van je brede kennis van de vakliteratuur, je doorzettingsvermogen en je kritische blik. Ook ben ik je dankbaar voor de ontelbare keren dat je mij heel snel van feedback voorzag, soms vanuit bijzondere oorden. Veel dank voor dit alles.

Beste David, je gedrevenheid, enthousiasme en de wijze waarop je mensen verenigt en weet te stimuleren tot een bijzondere samenwerking is inspirerend. Ik heb veel geleerd van jouw visie op wetenschappelijk onderzoek in de klinische praktijk en de wijze waarop je het initieerde en uitvoerde. Veel dank voor je stimulerende adviezen in het begin van het traject en je supervisie gedurende het promotietraject waarmee je me bleef uitdagen om het nog beter te doen.

Ik wil graag de leden van de leescommissie bedanken, Prof. dr. Arnoud Arntz, Prof. dr. Harald Merckelbach, Prof. dr. Chijs van Nieuwenhuizen, Prof. dr. Henk Nijman en dr. Vivienne de Vogel voor de bereidwilligheid om dit proefschrift te beoordelen.

Het wetenschappelijk onderzoek waarop dit proefschrift is gebaseerd, heb ik als medewerker van het forensisch psychiatrisch centrum de Rooyse Wissel mogen uitvoeren. In het bijzonder wil ik Sanne Verwaaijen en (voormalig Raad van Bestuur) Toine de Beer bedanken voor het bieden van deze mogelijkheid. Daarnaast wil ik mijn oud-collega's bedanken voor de prettige samenwerking en in het bijzonder, Maaike Cima en Franca Eurlings-Tonnaer. Dank voor jullie steun tijdens het schrijven van dit proefschrift.

Ik dank ook de vele patiënten die hebben deelgenomen aan het onderzoek of waarvan we gegevens uit hun straf- en verpleegdossier mochten gebruiken voor het onderzoek. In het bijzonder wil ik "Andy" bedanken die we jarenlang hebben mogen volgen voor de case studie, ook na zijn ontslag uit de TBS.

Cris de Bouter en Otto Pijpker, jullie zijn twee fantastische paranimfen.

Lieve Grietje, jou ben ik de meeste dank verschuldigd. Dank je voor je begrip tijdens het schrijven van dit proefschrift, vooral als het weer eens avond- en/of weekendwerk was en soms zelfs tijdens onze vakanties. Bovenal, dank ik je voor je liefde en je onvoorwaardelijke steun. Eigenlijk is dit proefschrift ook een beetje van jou. 
ABOUT THE AUTHOR

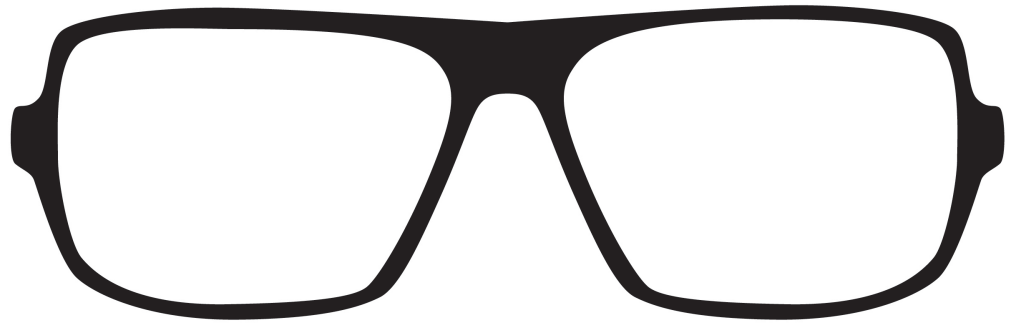

- 0 
Farid Chakhssi was born on August 25th 1974 in Venray, the Netherlands. After graduating from Jerusalem High School in Venray, he was enlisted in the military and served in peace keeping missions in the former Yugoslavia. Upon completing his service, he moved to Nijmegen where he began studying Psychology at Radboud University in 1997. During his study, he specialized in the area of forensic psychology by following several courses at the Faculty of Law, and by conducting his clinical and research internship at the Pompestichting, a forensic psychiatric hospital in Nijmegen. In March 2002, he obtained his master degree (met genoegen) in Clinical Psychology. From February 2002 to March 2013, he worked for the forensic psychiatric hospital 'de Rooyse Wissel'. During his time at the hospital, he conducted many psychological assessments, was responsible for successfully implementing several instruments into the clinical practice including the Behavioural Status Index and the Psychopathy Checklist-Revised and advised the board of directors on policy regarding violence risk assessment. Also, he was a member of the internal leave assessment committee where he advised clinicians on risk assessment and -management of patients during leave. On a national level, he worked together with other researchers in several projects, facilitated by the Dutch Expertise Centre for Forensic Psychiatry, aimed at innovation in the Dutch forensic field, such as the development of a national database for risk assessment scores of TBS patients. Furthermore, he contributed to the revised version of the Historisch Klinisch Toekomst-30 (HKT-R), and participated in the Dutch translation of the Historical Clinical Risk-management-20 version 3 (HCR-20 V3), the two most widely used risk assessment instruments in the Netherlands. As of 2008, he started his dissertation research under supervision of professor dr. Corine de Ruiter and professor dr. David Bernstein from the department of Clinical Psychological Science at Maastricht University, which resulted in the present thesis. Currently, he is on sabbatical leave exploring the east of the Netherlands. As of January 2014, he will start as a consultant for Bureau Apeneus. 